\title{
Synthesis and Reactions of 3,3-Difluoro-2-exo-Methylidene Indolines
}

\author{
Nicolas Zeidan, Matthew Zambri, Sven Unger, Christian Dank, Alexa Torelli, Bijan Mirabi, Mark Lautens* \\ <mark.lautens@utoronto.ca> \\ Department of Chemistry, University of Toronto, 80 St. George Street, \\ Toronto, Ontario, M5S 3H6, Canada
}

\section{Supporting Information}

Table of Contents

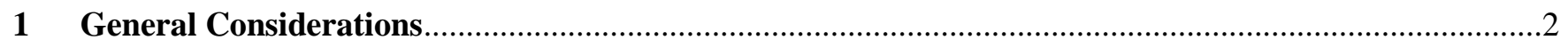

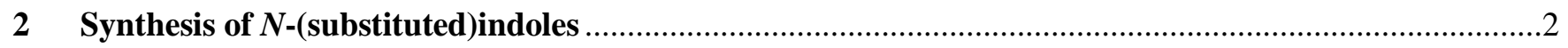

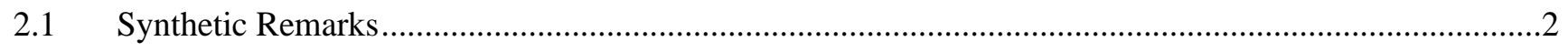

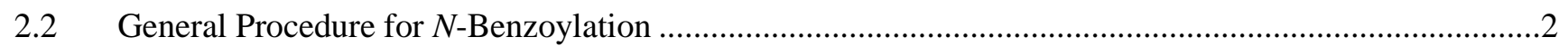

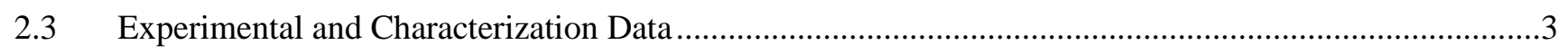

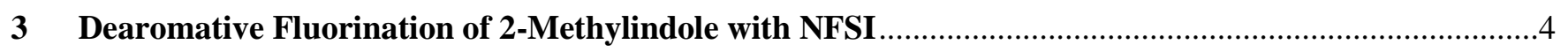

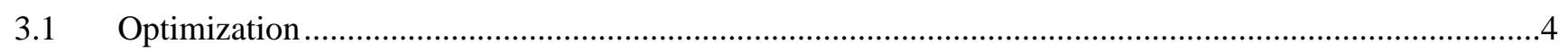

3.2 General Procedure for the Small Scale Difluorination of Indoles with NFSI .....................................4

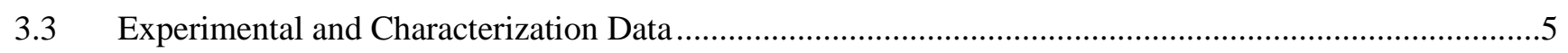

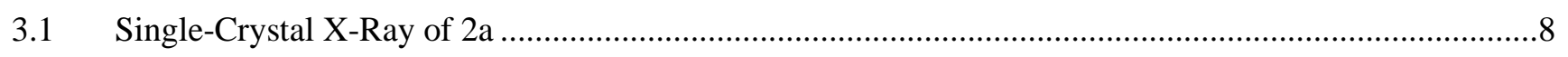

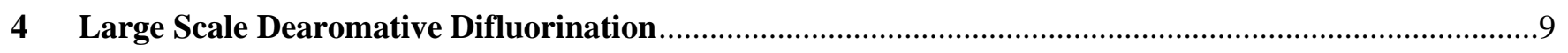

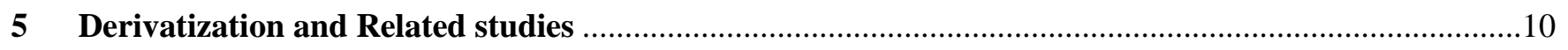

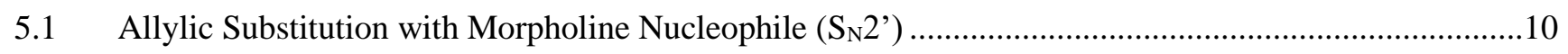

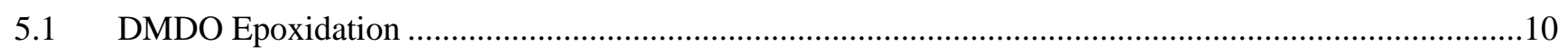

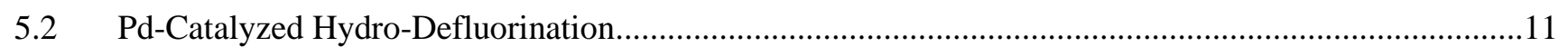

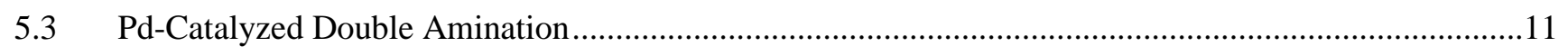

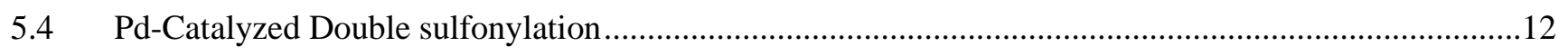

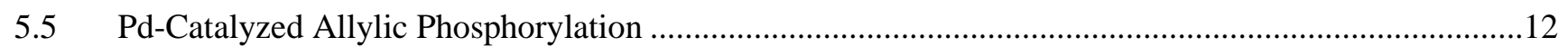




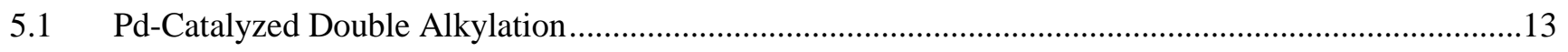

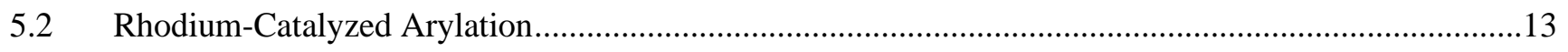

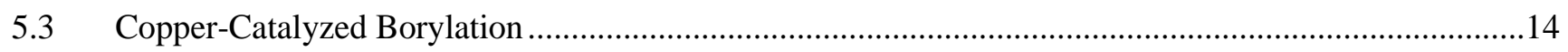

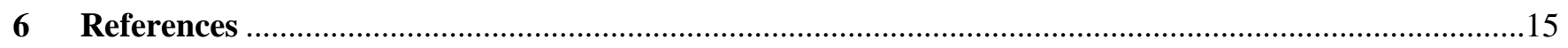

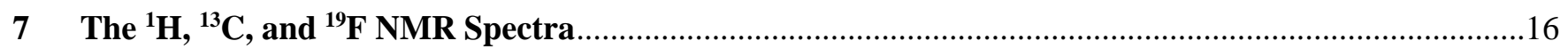

\section{General Considerations}

Metal catalyzed reactions were run under an atmosphere of argon in oven or flame dried 2-dram vials with open-top caps fitted with a Teflon septum. Reactions were monitored by thin-layer chromatography (TLC) on EMD Silica Gel 60 F254 plates and visualized under UV light or by immersion in iodine on silica stain. Flash column chromatography was performed on Silicycle 40-60 $\mu \mathrm{m}$ silica gel. PhMe was distilled over CaH. All reagents and organic building blocks were purchased from commercial supplier (Sigma Aldrich, Strem, Alfa Aesar, TCI, Combiblocks) and used without further purification.

${ }^{1} \mathrm{H}$ and ${ }^{13} \mathrm{C}$ NMR spectra were obtained on the Agilent DD2 500 equipped with a $5 \mathrm{~mm}$ Xsens Cold Probe. The spectra were internally referenced to the solvent peak. ${ }^{19} \mathrm{~F}$ and ${ }^{31} \mathrm{P}$ NMR spectra were obtained on the Varian Mercury 400 or 500 operating at 470 or $564 \mathrm{MHz}$. Measurements were taken at $296 \mathrm{~K}$ and chemical shifts are reported in parts per million (ppm). Data is reported in the following format: chemical shift ( $\delta \mathrm{ppm})$, multiplicity (s = singlet, $\mathrm{d}=$ doublet, $\mathrm{t}=$ triplet, $\mathrm{q}=$ quartet, $\mathrm{m}=$ multiplet $)$, coupling constant $(\mathrm{Hz})$, integration. High resolution mass spectra (HRMS) were obtained on a Micromass 70S-250 spectrometer (EI) or an ABI/Sciex QStar Mass Spectrometer (ESI) or a JEOL AccuTOF model JMS-T1000LC mass spectrometer equipped with an IONICS® Direct Analysis in real Time (DART) ion source.

\section{Synthesis of $N$-(substituted)indoles}

\subsection{Synthetic Remarks}

Compounds 1 were synthesized according to literature procedure, summarized in section 2.2, and characterization data for known compounds matched the literature data. ${ }^{1}$

\subsection{General Procedure for $N$-Benzoylation}

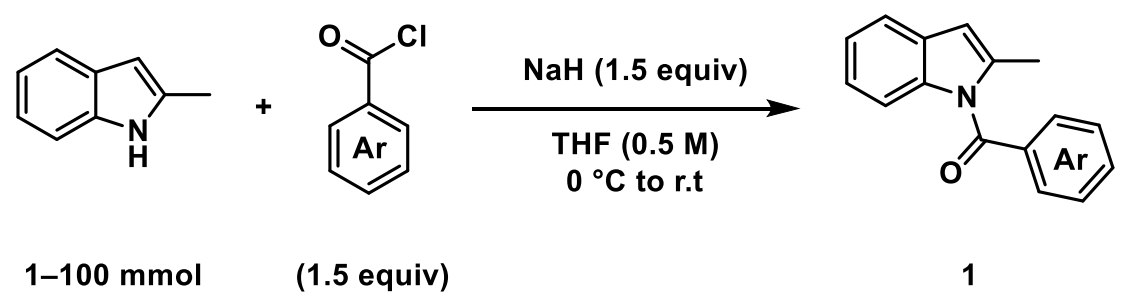

To a solution of indole $(1-100 \mathrm{mmol})$ in THF $(0.5 \mathrm{M})$, was added $\mathrm{NaH}(60 \%$ dispersion in mineral oil, 1.2 equiv) slowly, in a water bath as a heat sink. After 30 minutes of stirring, the corresponding acyl-chloride (1.2-2 equiv) in THF (1 M) was added over 1 minute. The reaction was stirred for $2 \mathrm{hr}$ and quenched with $\mathrm{NH}_{4} \mathrm{Cl}$ (Caution: 
exothermic and vigorous reaction with left over $\mathrm{NaH}$ ). The aqueous layer was extracted with EtOAc, and the organic layer was washed with $\mathrm{NaHCO}_{3}$ (aq) and brine. After the solvent was removed, the crude mixture was columned on silica gel using relatively non-polar conditions to remove all traces of indole which typically elute rapidly (typically seen as a pink-red color upon standing of pure material).

\subsection{Experimental and Characterization Data}

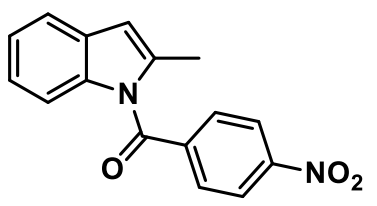

(2-methyl-1H-indol-1-yl)(4-nitrophenyl)methanone (1d) - The compound was synthesized according to general procedure 2.2. The product was purified by flash column chromatography using pentanes-EtOAc-NEt 3 (100:1:1 v:v) as the mobile phase and was isolated as a yellow solid (204 mg, $0.728 \mathrm{mmol}, 14 \%) .{ }^{\mathbf{1}} \mathbf{H}$ NMR (400 MHz, $\left.\mathrm{CDCl}_{3}\right) \delta 8.39-8.31(\mathrm{~m}, 2 \mathrm{H}), 7.91-7.84(\mathrm{~m}, 2 \mathrm{H}), 7.48(\mathrm{dt}, J=7.8,1.0 \mathrm{~Hz}, 1 \mathrm{H}), 7.18$ (ddd, $J=7.9,7.2,1.0 \mathrm{~Hz}, 1 \mathrm{H}), 7.05(\mathrm{ddd}, J=8.4,7.2,1.3 \mathrm{~Hz}, 1 \mathrm{H}), 6.95(\mathrm{dq}, J=8.4,0.9 \mathrm{~Hz}, 1 \mathrm{H}), 6.47$ (p, $J=1.1$ $\mathrm{Hz}, 1 \mathrm{H}), 2.41(\mathrm{~d}, J=1.2 \mathrm{~Hz}, 3 \mathrm{H}) .{ }^{13} \mathbf{C} \mathbf{N M R}\left(100 \mathrm{MHz}, \mathrm{CDCl}_{3}\right) \delta 167.8,150.2,141.3,137.7,136.9,130.6,129.9$, 124.1, 123.5, 123.4, 120.3, 114.4, 110.0, 16.2. IR (thin film, $\mathrm{cm}^{-1}$ ) 3399, 3113, 3070, 3052, 2926, 2858, 1686, 1602, 1454, 1525, 1193, 804, 708. HRMS (DART, M+H) Calculated for $\mathrm{C}_{16} \mathrm{H}_{13} \mathrm{~N}_{2} \mathrm{O}_{3}$ 281.0926, found 281.0928.

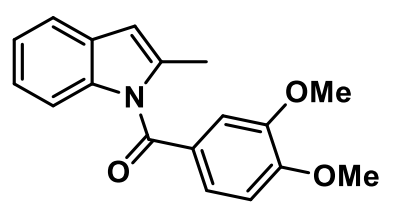

(3,4-dimethoxyphenyl)(2-methyl-1H-indol-1-yl)methanone (1g) - The compound was synthesized according to general procedure 2.2. The product was purified by flash column chromatography using pentanes-EtOAc-NEt $3(30: 1: 1 \mathrm{v}: \mathrm{v})$ as the mobile phase and was isolated as a white solid $(2.34 \mathrm{~g}, 7.92 \mathrm{mmol}, 72 \%) .{ }^{1} \mathbf{H}$ NMR $(400 \mathrm{MHz}$, $\left.\mathrm{CDCl}_{3}\right) \delta 7.45-7.40(\mathrm{~m}, 1 \mathrm{H}), 7.38(\mathrm{dq}, J=8.3,0.9 \mathrm{~Hz}, 1 \mathrm{H}), 7.20-7.12(\mathrm{~m}, 2 \mathrm{H}), 7.11$ - $7.04(\mathrm{~m}, 2 \mathrm{H}), 6.96(\mathrm{dd}, J=7.5,1.7 \mathrm{~Hz}, 1 \mathrm{H}), 6.36(\mathrm{t}, J=1.0 \mathrm{~Hz}, 1 \mathrm{H}), 3.92(\mathrm{~s}, 3 \mathrm{H}), 3.77(\mathrm{~s}, 3 \mathrm{H}), 2.28(\mathrm{~d}, J=1.2$ $\mathrm{Hz}, 3 \mathrm{H}) .{ }^{13} \mathbf{C}$ NMR $\left(100 \mathrm{MHz}, \mathrm{CDCl}_{3}\right) \delta 167.7,153.2,146.8,137.9,137.2,132.1,129.9,124.7,123.5,123.3,120.2$, 119.7, 115.1, 115.1, 109.7, 61.8, 56.1, 16.3. IR (thin film, $\mathrm{cm}^{-1}$ ) 2981, 2927, 2840, 1668, 1592, 1574, 1456, 1441, 1361, 984, 749. HRMS (DART, M+H) Calculated for $\mathrm{C}_{18} \mathrm{H}_{18} \mathrm{NO}_{3} 296.1281$, found 296.1282.

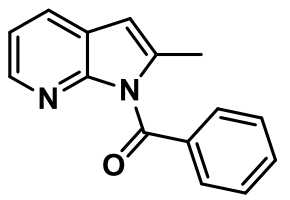

(3,4-dimethoxyphenyl)(2-methyl-1H-indol-1-yl)methanone (lh) - The compound was synthesized according to general procedure 2.2. The product was purified by flash column chromatography using hexanes-EtOAc-DCM (20:1:1 v:v) as the mobile phase and was isolated as a white solid (591 mg, $2.50 \mathrm{mmol}, 74 \%) .{ }^{1} \mathbf{H} \mathbf{~ N M R}\left(500 \mathrm{MHz}, \mathrm{CDCl}_{3}\right) \delta 8.03$ (dd, $J=4.8,1.6 \mathrm{~Hz}, 1 \mathrm{H}), 7.80-7.74(\mathrm{~m}, 3 \mathrm{H}), 7.65-7.59(\mathrm{~m}, 1 \mathrm{H}), 7.48-7.42(\mathrm{~m}, 2 \mathrm{H}), 7.05$ (dd, $J=7.7,4.8 \mathrm{~Hz}, 1 \mathrm{H}), 6.39(\mathrm{q}, J=1.1 \mathrm{~Hz}, 1 \mathrm{H}), 2.59(\mathrm{~d}, J=1.2 \mathrm{~Hz}, 3 \mathrm{H}) .{ }^{13} \mathbf{C} \mathbf{N M R}\left(125 \mathrm{MHz}, \mathrm{CDCl}_{3}\right) \delta 169.7,149.7$, $142.8,139.1,135.0,133.4,130.9,128.4,127.6,121.6,118.3,104.8,15.3$. IR (thin film, $\mathrm{cm}^{-1}$ ) 3013, 2960, 2931, 1686, 1596, 1560, 1402, 1305, 1251, 904, 812. HRMS (DART, M+H) Calculated for $\mathrm{C}_{15} \mathrm{H}_{13} \mathrm{~N}_{2} \mathrm{O} 237.1022$, found 237.1026 . 


\section{Dearomative Fluorination of 2-Methylindole with NFSI}

\subsection{Optimization}

Other solvents were tolerated (Table 1, entry 1-3) however, the reaction was best in THF (Table 1, entry 4). Notably, DCM gave no product, possibly due to the background amination reaction reported by Yang. ${ }^{2}$ Dropping the temperature resulted in diminished yield (table 1, entry 5). Concentrating the reaction increased the yield (table 1, entry 6) however, further increasing the concentration to $0.5 \mathrm{M}$ resulted in the immediate appearance of a black colour and decomposition ensued (table 1, entry 7). A wide range of additives, organic bases, inorganic bases, and acids were screened, and we found that the addition of $\mathrm{NH}_{4} \mathrm{Cl}$ increased the yield to $60 \%$ (Table 1, entry 8).

Table 1. Effects of Reaction Parameters in the Dearomative Arylation/Heteroarylation of Indoles ${ }^{a}$

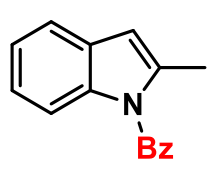

1 a

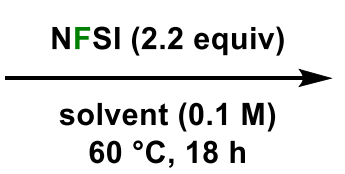

$60^{\circ} \mathrm{C}, 18 \mathrm{~h}$

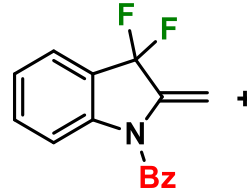

$2 \mathbf{a}$

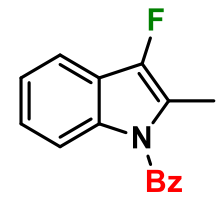

5

$\begin{array}{lllll}\text { entry } & \text { solvent } & \text { variation } & \mathbf{2 a}(\%) & \mathbf{5}(\%) \\ 1 & \text { MTBE } & \text { none } & 32 & - \\ 2 & \text { DCM } & \text { none } & - & - \\ 3 & \text { MeCN } & \text { none } & 26 & - \\ 4 & \text { THF } & \text { none } & 47 & <5 \\ 5 & \text { THF } & 40^{\circ} \mathrm{C} & 37 & 15 \\ 6 & \text { THF } & 0.2 \mathrm{M} & 48 & 15 \\ 7 & \text { THF } & 0.5 \mathrm{M} & \text { decomp } & - \\ 8 & \text { THF } & 0.2 \mathrm{M} \text { and } 1 \text { equiv } \mathrm{NH}_{4} \mathrm{Cl} & 60 & -\end{array}$

${ }^{a}$ Reactions run on $0.2 \mathrm{mmol}$ scale and yields were determined by NMR using trifluorotoluene as an internal standard.

\subsection{General Procedure for the Small-Scale Difluorination of Indoles with NFSI}

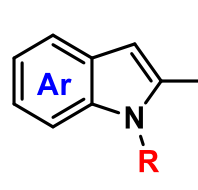

1

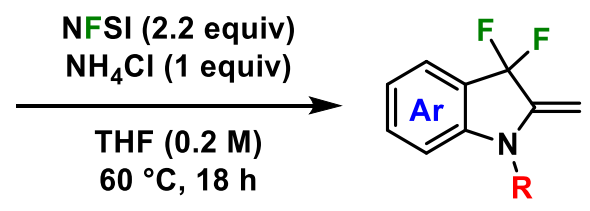

2

In an oven-dried 2-dram vial equipped with a open-top septum cap purging with an argon balloon was added substrate 1a $(0.2 \mathrm{mmol})$, NFSI $(0.44 . \mathrm{mmol})$, and $\mathrm{NH}_{4} \mathrm{Cl}(0.2 \mathrm{mmol})$. After a 10-minute purge, THF (1 $\left.\mathrm{mL}, 0.2 \mathrm{M}\right)$ was added. The balloon was removed, and the reaction was heated in an oil bath to $60{ }^{\circ} \mathrm{C}$, for $12-24 \mathrm{~h}$. Upon completion, the reaction was diluted with pentanes to crash out the polar biproducts and filtered through a silica plug, eluting with a 2:1 solvent mixture of pentanes- $\mathrm{Et}_{2} \mathrm{O}$. The reaction was concentrated and purified via silica gel chromatography. When required, analytically pure material was obtained by recrystallization in pentanes. 


\subsection{Experimental and Characterization Data}

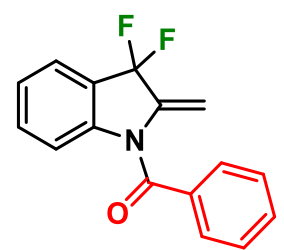

(3,3-difluoro-2-methyleneindolin-1-yl)(phenyl)methanone (2a) - Was synthesized according to general procedure 3.2. The product was purified by flash column chromatography using pentanes- $\mathrm{Et}_{2} \mathrm{O}(50: 1$ to $30: 1, \mathrm{v}: \mathrm{v})$ as the mobile phase and was isolated as a white solid (32.6 mg, $0.120 \mathrm{mmol}, 60 \%) .{ }^{1} \mathbf{H}$ NMR $\left(400 \mathrm{MHz}, \mathrm{CDCl}_{3}\right) \delta 7.68-7.60(\mathrm{~m}, 3 \mathrm{H}), 7.60-7.54$ (m, 1H), $7.53-7.44(\mathrm{~m}, 2 \mathrm{H}), 7.41-7.35(\mathrm{~m}, 2 \mathrm{H}), 7.22(\mathrm{ddd}, J=7.7,4.7,3.7 \mathrm{~Hz}, 1 \mathrm{H}), 5.43$ $(\mathrm{td}, J=3.0,2.6 \mathrm{~Hz}, 1 \mathrm{H}), 5.18(\mathrm{td}, J=3.6,2.6 \mathrm{~Hz}, 1 \mathrm{H}) .{ }^{13} \mathbf{C} \mathbf{N M R}\left(125 \mathrm{MHz}, \mathrm{CDCl}_{3}\right) \delta 168.8$, $142.8(\mathrm{t}, J=5.8 \mathrm{~Hz}), 142.6(\mathrm{t}, J=26.9 \mathrm{~Hz}), 135.2,132.9(\mathrm{t}, J=1.5 \mathrm{~Hz}), 132.2,129.0,128.5,124.8(\mathrm{t}, J=1.7 \mathrm{~Hz})$, $124.1,122.9(\mathrm{t}, J=24.8 \mathrm{~Hz}), 118.2(\mathrm{t}, J=240.6 \mathrm{~Hz}), 116.4,102.0(\mathrm{t}, J=3.3 \mathrm{~Hz}) .{ }^{19} \mathbf{F ~ N M R}\left(377 \mathrm{MHz}, \mathrm{CDCl}_{3}\right) \delta-$ 85.13. IR (thin film, cm ${ }^{-1}$ ) 3338, 3151, 3129, 3061, 3029, 1673, 1616, 1600, 1462, 1355, 1152, 1047, 767. HRMS (DART, M+H) Calculated for $\mathrm{C}_{16} \mathrm{H}_{12} \mathrm{~F}_{2} \mathrm{NO} 272.0887$, found 272.0892 .

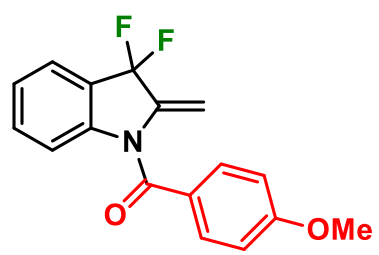

(3,3-difluoro-2-methyleneindolin-1-yl)(4-methoxyphenyl)methanone (2b) - Was synthesized according to general procedure 3.2 however on $1 \mathrm{mmol}$ scale. The product was purified by flash column chromatography using pentanes- $\mathrm{Et}_{2} \mathrm{O}(30: 1, \mathrm{v}: \mathrm{v})$ as the mobile phase and was isolated as a white solid (181 $\mathrm{mg}, 0.601 \mathrm{mmol}, 60 \%) .{ }^{1} \mathbf{H}$ NMR $\left(400 \mathrm{MHz}, \mathrm{CDCl}_{3}\right) \delta 7.67-7.58(\mathrm{~m}, 3 \mathrm{H}), 7.42-7.33(\mathrm{~m}, 2 \mathrm{H}), 7.22-7.16(\mathrm{~m}, 1 \mathrm{H}), 6.99$ $-6.92(\mathrm{~m}, 2 \mathrm{H}), 5.41(\mathrm{td}, J=3.2,2.5 \mathrm{~Hz}, 1 \mathrm{H}), 5.20(\mathrm{td}, J=3.6,2.5 \mathrm{~Hz}, 1 \mathrm{H}), 3.88(\mathrm{~s}, 3 \mathrm{H})$. ${ }^{13} \mathrm{C}$ NMR $\left(100 \mathrm{MHz}, \mathrm{CDCl}_{3}\right) \delta 168.4,163.0,143.2(\mathrm{t}, J=6.0 \mathrm{~Hz}), 143.0(\mathrm{t}, J=26.6 \mathrm{~Hz}), 132.9(\mathrm{t}, J=1.5 \mathrm{~Hz})$, $131.1,127.0,124.4(\mathrm{t}, J=1.7 \mathrm{~Hz}), 124.0,122.7(\mathrm{t}, J=24.8 \mathrm{~Hz}), 118.3(\mathrm{t}, J=239.9 \mathrm{~Hz}), 116.1,114.2,101.1(\mathrm{t}, J=$ $3.3 \mathrm{~Hz})$, 55.6. ${ }^{19} \mathbf{F}$ NMR $\left(377 \mathrm{MHz}, \mathrm{CDCl}_{3}\right) \delta$-85.04. IR (thin film, $\left.\mathrm{cm}^{-1}\right)$ 3130, 3012, 2937, 2842, 1736, 1671, 1604, 1578, 1469, 1252, 756. HRMS (DART, M+H) Calculated for $\mathrm{C}_{17} \mathrm{H}_{14} \mathrm{NO}_{2} \mathrm{~F}_{2} 302.0987$, found 302.0991.

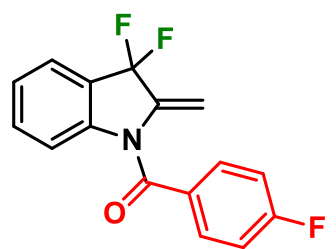

(3,3-difluoro-2-methyleneindolin-1-yl)(4-fluorophenyl)methanone $\quad(2 \mathrm{c}) \quad-\quad$ Was synthesized according to general procedure 3.2 with the following variations. The reaction was run on $0.5 \mathrm{mmol}$ scale. The product contained two unknown, inseparable biproducts. The yield was measured by proton NMR using $10.6 \mathrm{mg}$ of TMB as an internal standard $(0.197 \mathrm{mmol}, 39 \%) .{ }^{1} \mathbf{H}$ NMR $\left(400 \mathrm{MHz}, \mathrm{CDCl}_{3}\right) \delta 7.72-7.61(\mathrm{~m}, 3 \mathrm{H}), 7.43-7.38(\mathrm{~m}$, 2H), $7.26-7.21(\mathrm{~m}, 1 \mathrm{H}), 7.20-7.13(\mathrm{~m}, 2 \mathrm{H}), 5.45(\mathrm{td}, J=2.9,2.9 \mathrm{~Hz}, 1 \mathrm{H}), 5.17(\mathrm{td}, J=$ 3.3, $2.9 \mathrm{~Hz}, 1 \mathrm{H}) .{ }^{13} \mathbf{C} \mathbf{N M R}\left(100 \mathrm{MHz}, \mathrm{CDCl}_{3}\right) \delta 167.4,164.9(\mathrm{~d}, J=253.8 \mathrm{~Hz}), 143.0-142.3(\mathrm{~m}), 132.8(\mathrm{t}, J=$ $1.5 \mathrm{~Hz}), 131.2(\mathrm{~d}, J=8.9 \mathrm{~Hz}), 131.0(\mathrm{~d}, J=3.5 \mathrm{~Hz}), 124.8(\mathrm{t}, J=1.7 \mathrm{~Hz}), 124.0,122.7(\mathrm{t}, J=24.8 \mathrm{~Hz}), 118.1(\mathrm{t}, J$ $=240.1 \mathrm{~Hz}), 116.1(\mathrm{~d}, J=8.1 \mathrm{~Hz}), 116.0,101.7(\mathrm{t}, J=3.3 \mathrm{~Hz}) .{ }^{19} \mathbf{F} \mathbf{N M R}\left(377 \mathrm{MHz}, \mathrm{CDCl}_{3}\right) \delta-85.39(\mathrm{~s}, 2 \mathrm{~F}),-$ 105.72 (td, $J=8.4,7.9,4.2 \mathrm{~Hz}, 1 \mathrm{~F})$. IR (thin film, $\mathrm{cm}^{-1}$ ) 3125, 3068, 3015, 2969, 3927, 1664, 1615, 1602, 1482, 1350, 1270, 1196, 1048. HRMS (DART, M+H) Calculated for $\mathrm{C}_{16} \mathrm{H}_{11} \mathrm{NOF}_{3} 290.0787$, found 290.0783.

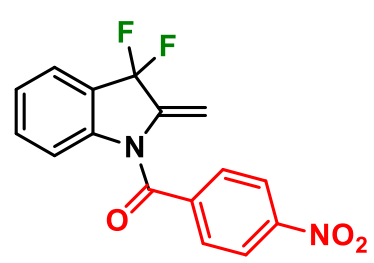

(3,3-difluoro-2-methyleneindolin-1-yl)(4-nitrophenyl)methanone (2d) - Was synthesized according to general procedure 3.2. The product was purified by flash column chromatography using pentanes- $\mathrm{Et}_{2} \mathrm{O}$ (50:1 to $\left.30: 1, \mathrm{v}: \mathrm{v}\right)$ as the mobile phase and was isolated as a yellow solid $(23.4 \mathrm{mg}, 0.0740 \mathrm{mmol}, 37 \%) .{ }^{1} \mathbf{H}$ NMR $(400 \mathrm{MHz}$, $\left.\mathrm{CDCl}_{3}\right) \delta 8.38-8.31(\mathrm{~m}, 2 \mathrm{H}), 7.84-7.77(\mathrm{~m}, 2 \mathrm{H}), 7.72-7.63(\mathrm{~m}, 1 \mathrm{H}), 7.43(\mathrm{~d}, J=4.2$ $\mathrm{Hz}, 2 \mathrm{H}), 7.28(\mathrm{dd}, J=8.5,4.8 \mathrm{~Hz}, 1 \mathrm{H}), 5.50(\mathrm{td}, J=2.9,2.9 \mathrm{~Hz}, 1 \mathrm{H}), 5.14(\mathrm{td}, J=3.2$, $2.9 \mathrm{~Hz}, 1 \mathrm{H}) .{ }^{13} \mathbf{C}$ NMR $\left(100 \mathrm{MHz}, \mathrm{CDCl}_{3}\right) \delta 166.2,149.7,142.4(\mathrm{t}, J=27.3 \mathrm{~Hz}), 142.1(\mathrm{t}, J=5.8 \mathrm{~Hz}), 140.7,133.2$ $(\mathrm{t}, J=1.5 \mathrm{~Hz}), 129.6,125.6(\mathrm{t}, J=1.7 \mathrm{~Hz}), 124.3,123.1(\mathrm{t}, J=24.8 \mathrm{~Hz}), 117.9(\mathrm{t}, J=240.5 \mathrm{~Hz}), 116.4,103.0(\mathrm{t}, J$ 
$=3.1 \mathrm{~Hz}) .{ }^{19} \mathbf{F}$ NMR $\left(377 \mathrm{MHz}, \mathrm{CDCl}_{3}\right) \delta$-86.03. IR (thin film, $\left.\mathrm{cm}^{-1}\right) 3111,2981,1674,1615,1601,1526,1472$, 1346, 1294, 762. HRMS (DART, M+H) Calculated for $\mathrm{C}_{16} \mathrm{H}_{11} \mathrm{~F}_{2} \mathrm{~N}_{2} \mathrm{O}_{3} 317.0738$, found 317.0743.

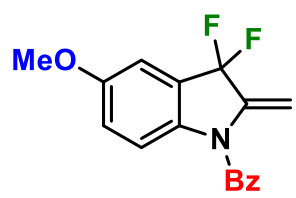

(3,3-difluoro-5-methoxy-2-methyleneindolin-1-yl)(phenyl)methanone (2e) - Was synthesized according to general procedure 3.2. The product was purified by flash column chromatography using pentanes- $\mathrm{Et}_{2} \mathrm{O}(50: 1$ to $30: 1, \mathrm{v}: \mathrm{v})$ as the mobile phase and was isolated as a white solid (26.5 mg, $0.0880 \mathrm{mmol}, 44 \%) .{ }^{1} \mathbf{H} \mathbf{~ N M R}\left(400 \mathrm{MHz}, \mathrm{CDCl}_{3}\right) \delta 7.63-7.52$ $(\mathrm{m}, 3 \mathrm{H}), 7.50-7.43(\mathrm{~m}, 2 \mathrm{H}), 7.34(\mathrm{dt}, J=9.1,1.5 \mathrm{~Hz}, 1 \mathrm{H}), 7.14(\mathrm{dt}, J=2.8,1.4 \mathrm{~Hz}, 1 \mathrm{H})$, $6.93(\mathrm{ddt}, J=9.1,2.6,1.1 \mathrm{~Hz}, 1 \mathrm{H}), 5.41(\mathrm{td}, J=2.9 \mathrm{~Hz}, 1 \mathrm{H}), 5.14(\mathrm{td}, J=3.3 \mathrm{~Hz}, 1 \mathrm{H}), 3.83(\mathrm{~s}, 3 \mathrm{H}) .{ }^{13} \mathbf{C} \mathbf{N M R}(125$ $\left.\mathrm{MHz} \mathrm{CDCl}_{3}\right) \delta 166.2,149.7,142.4(\mathrm{t}, J=27.3 \mathrm{~Hz}), 142.1(\mathrm{t}, J=5.8 \mathrm{~Hz}), 140.7,133.2(\mathrm{t}, J=1.5 \mathrm{~Hz}), 129.6,125.6$ $(\mathrm{t}, J=1.7 \mathrm{~Hz}), 124.3,123.1(\mathrm{t}, J=24.8 \mathrm{~Hz}), 117.9(\mathrm{t}, J=240.5 \mathrm{~Hz}), 116.4,103.0(\mathrm{t}, J=3.1 \mathrm{~Hz}) .{ }^{19} \mathbf{F}$ NMR $(377$ $\mathrm{MHz}, \mathrm{CDCl}_{3}$ ) $\delta$-85.45. IR (thin film, $\mathrm{cm}^{-1}$ ) 3137, 3068, 3028, 3012, 2967, 2921, 2851, 2835, 1655, 1486, 1361, 1220, 1047. HRMS (DART, M+H) Calculated for $\mathrm{C}_{17} \mathrm{H}_{14} \mathrm{~F}_{2} \mathrm{NO}_{2} 302.0993$, found 302.0995.

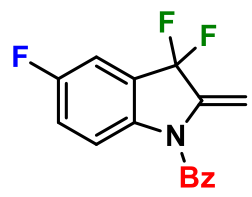

phenyl(3,3,5-trifluoro-2-methyleneindolin-1-yl)methanone (2f) - Was synthesized according to general procedure 3.2 however on $1 \mathrm{mmol}$ scale. The product was purified by flash column chromatography using pentanes- $\mathrm{Et}_{2} \mathrm{O}(50: 1, \mathrm{v}: \mathrm{v})$ as the mobile phase and was isolated as a white solid (169 mg, $0.584 \mathrm{mmol}, 58 \%) .{ }^{1} \mathbf{H}$ NMR $\left(400 \mathrm{MHz}, \mathrm{CDCl}_{3}\right) \delta 7.66-7.52(\mathrm{~m}, 3 \mathrm{H})$, $7.52-7.43(\mathrm{~m}, 2 \mathrm{H}), 7.37-7.30(\mathrm{~m}, 1 \mathrm{H}), 7.13(\mathrm{td}, J=8.9,2.8 \mathrm{~Hz}, 1 \mathrm{H}), 5.41(\mathrm{td}, J=3.1,2.9 \mathrm{~Hz}$, $1 \mathrm{H}), 5.01(\mathrm{td}, J=3.3,2.9 \mathrm{~Hz}, 1 \mathrm{H}) \cdot{ }^{13} \mathbf{C}$ NMR $\left(125 \mathrm{MHz}, \mathrm{CDCl}_{3}\right) \delta 168.3,157.1(\mathrm{t}, J=1.8 \mathrm{~Hz}), 142.8(\mathrm{t}, J=26.8$ $\mathrm{Hz}), 136.4(\mathrm{t}, J=6.0 \mathrm{~Hz}), 135.4,131.9,128.9,128.3,123.8(\mathrm{t}, J=24.4 \mathrm{~Hz}), 119.5(\mathrm{t}, J=1.6 \mathrm{~Hz}), 118.2(\mathrm{t}, J=$ $240.4 \mathrm{~Hz}), 117.7,107.9,102.1(\mathrm{t}, J=3.3 \mathrm{~Hz}), 55.9 .{ }^{19} \mathbf{F} \mathbf{N M R}\left(377 \mathrm{MHz}, \mathrm{CDCl}_{3}\right) \delta-85.57(\mathrm{~s}, 2 \mathrm{~F}),-116.44(\mathrm{dd}, J=$ $7.3 \mathrm{~Hz}, 1 \mathrm{~F})$. IR (thin film, $\mathrm{cm}^{-1}$ ) 3141, 3065, 3017, 2926, 2855, 1666, 1482, 1268, 1188. HRMS (DART, M+H) Calculated for $\mathrm{C}_{16} \mathrm{H}_{11} \mathrm{~F}_{3} \mathrm{NO} 290.0793$, found 290.0790 .

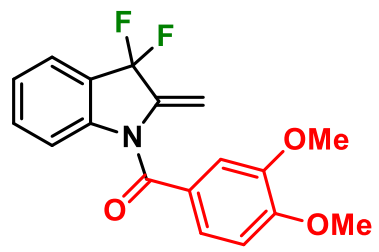

(3,3-difluoro-2-methyleneindolin-1-yl)(3,4-dimethoxyphenyl)methanone $\quad(2 \mathrm{~g}) \quad-$ Was synthesized according to general procedure 3.2. The product was purified by flash column chromatography using pentanes- $\mathrm{Et}_{2} \mathrm{O}$ (50:1 to 30:1, v:v) as the mobile phase and was isolated as a white solid $(39.7 \mathrm{mg}, 0.120 \mathrm{mmol}, 33 \%) .{ }^{1} \mathbf{H}$ NMR (400 MHz, $\left.\mathrm{CDCl}_{3}\right) \delta 7.62(\mathrm{dt}, J=7.6,1.0 \mathrm{~Hz}, 1 \mathrm{H}), 7.41-7.28(\mathrm{~m}, 2 \mathrm{H}), 7.24-7.18(\mathrm{~m}, 1 \mathrm{H}), 7.17$ $-7.13(\mathrm{~m}, 1 \mathrm{H}), 7.07(\mathrm{dd}, J=8.3,1.5 \mathrm{~Hz}, 1 \mathrm{H}), 6.95(\mathrm{dd}, J=7.6,1.5 \mathrm{~Hz}, 1 \mathrm{H}), 5.43(\mathrm{~d}, J$ $=2.3 \mathrm{~Hz}, 1 \mathrm{H}), 5.34(\mathrm{~s}, 1 \mathrm{H}), 3.90(\mathrm{~s}, 3 \mathrm{H}), 3.80(\mathrm{~s}, 3 \mathrm{H}) .{ }^{13} \mathbf{C} \mathbf{N M R}\left(125 \mathrm{MHz}, \mathrm{CDCl}_{3}\right) \delta 166.4,153.1,146.0,142.4$ $(\mathrm{t}, J=5.9 \mathrm{~Hz}), 142.1(\mathrm{t}, J=26.9 \mathrm{~Hz}), 133.0(\mathrm{t}, J=1.5 \mathrm{~Hz}), 131.0,125.1(\mathrm{t}, J=1.9 \mathrm{~Hz}), 125.0,124.0,123.2(\mathrm{t}, J=$ $24.8 \mathrm{~Hz}), 119.5,118.1(\mathrm{t}, J=239.7 \mathrm{~Hz}), 116.4,114.9,102.3,61.7,56.1 .{ }^{19} \mathbf{F}$ NMR $\left(377 \mathrm{MHz}, \mathrm{CDCl}_{3}\right) \delta-82.94(\mathrm{~d}$, $J=270.8 \mathrm{~Hz}$ ), $-86.23\left(\mathrm{~d}, J=271.5 \mathrm{~Hz}\right.$ ). IR (thin film, $\mathrm{cm}^{-1}$ ) 3012, 2938, 2839, 1680, 1615, 1398, 1582, 1471, 1358, 1290, 1270, 1048, 751. HRMS (DART, M+H) Calculated for $\mathrm{C}_{18} \mathrm{H}_{16} \mathrm{~F}_{2} \mathrm{NO}_{3} 332.1093$, found 332.1088.

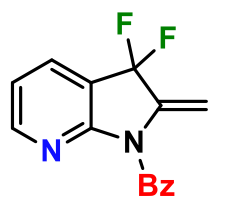

(3,3-difluoro-2-methylene-2,3-dihydro-1H-pyrrolo[2,3-b]pyridin-1-yl)(phenyl)methanone (2h) - Was synthesized according to general procedure 3.2. The product was purified by flash column chromatography using pentanes-EtOAc (30:1, v:v) as the mobile phase and was isolated as a white solid (18.1 mg, $0.0665 \mathrm{mmol}, 33 \%) .{ }^{1} \mathbf{H}$ NMR $\left(400 \mathrm{MHz}, \mathrm{CDCl}_{3}\right) \delta 8.14(\mathrm{ddt}, \mathrm{J}=5.0$, 1.9, $1.0 \mathrm{~Hz}, 1 \mathrm{H}), 7.93(\mathrm{dq}, \mathrm{J}=7.6,1.5 \mathrm{~Hz}, 1 \mathrm{H}), 7.68-7.59(\mathrm{~m}, 2 \mathrm{H}), 7.55$ (ddt, J = 7.9, 7.0, 1.3 Hz, $1 \mathrm{H}), 7.47-7.37(\mathrm{~m}, 2 \mathrm{H}), 7.06(\mathrm{dd}, \mathrm{J}=7.6,5.0 \mathrm{~Hz}, 1 \mathrm{H}), 6.27(\mathrm{td}, \mathrm{J}=4.1,2.2 \mathrm{~Hz}, 1 \mathrm{H}), 5.63(\mathrm{td}, \mathrm{J}=3.4,2.1 \mathrm{~Hz}$, 1H). ${ }^{13} \mathrm{C} \mathrm{NMR}\left(125 \mathrm{MHz}, \mathrm{CDCl}_{3}\right) \delta 168.8,155.4(\mathrm{t}, J=6.0 \mathrm{~Hz}), 152.0(\mathrm{t}, J=1.7 \mathrm{~Hz}), 141.2(\mathrm{t}, J=26.6 \mathrm{~Hz}), 135.5$, $133.3,132.2,129.1,128.2,119.6(\mathrm{t}, J=1.4 \mathrm{~Hz}), 116.3(\mathrm{t}, J=241.0 \mathrm{~Hz}), 115.8(\mathrm{t}, J=25.7 \mathrm{~Hz}), 102.2(\mathrm{t}, J=3.2$ 
Hz). ${ }^{19}$ F NMR (377 MHz, $\left.\mathrm{CDCl}_{3}\right) \delta$-84.64. IR (thin film, $\mathrm{cm}^{-1}$ ) 3029, 2961, 2924, 2856, 1684, 1594, 1473, 1449, 1420, 1272, 1059, 902. HRMS (DART, $\mathrm{M}+\mathrm{H}$ ) Calculated for $\mathrm{C}_{19} \mathrm{H}_{11} \mathrm{~F}_{2} \mathrm{~N}_{2} \mathrm{O} 273.0834$, found 273.0835. 


\subsection{Single-Crystal X-Ray of 2a}

\section{Datablock: d19161_a}

\begin{tabular}{|c|c|c|}
\hline Bond precision: & $C-C=0.0032 \mathrm{~A}$ & Wavelength $=0.71073$ \\
\hline Cell: & $\begin{array}{ll}3(19) \quad b=5.7406(6) \\
\text { beta }=102.044(6)\end{array}$ & $\begin{array}{l}c=15.530(2) \\
\text { gamma }=90\end{array}$ \\
\hline \multicolumn{3}{|c|}{ Temperature: $150 \mathrm{~K}$} \\
\hline & Calculated & Reported \\
\hline Volume & $1268.6(3)$ & $1268.6(3)$ \\
\hline Space group & P $21 / n$ & P 21/n \\
\hline Hall group & $-P 2 y n$ & $-P 2 y n$ \\
\hline Moiety formula & \multirow{2}{*}{ 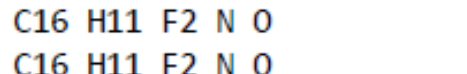 } & $\mathrm{C} 16 \mathrm{H} 11 \mathrm{~F} 2 \mathrm{~N} 0$ \\
\hline Sum formula & & $\mathrm{C} 16 \mathrm{H} 11 \mathrm{~F} 2 \mathrm{~N} 0$ \\
\hline $\mathrm{Mr}$ & 271.26 & 271.26 \\
\hline $\mathrm{Dx}, \mathrm{g} \mathrm{cm}-3$ & 1.420 & 1.420 \\
\hline $\mathrm{z}$ & 4 & 4 \\
\hline Mu $(m m-1)$ & 0.109 & 0.109 \\
\hline F000 & 560.0 & 560.0 \\
\hline F000' & 560.33 & \\
\hline$h, k, 1$ max & $17,6,18$ & $17,6,18$ \\
\hline Nref & 2232 & 2232 \\
\hline Tmin, Tmax & $0.987,0.996$ & $0.647,0.746$ \\
\hline Tmin & \multicolumn{2}{|l|}{0.981} \\
\hline \multicolumn{3}{|c|}{$\begin{array}{l}\text { Correction method }=\# \text { Reported T Limits: Tmin }=0.647 \text { Tmax }=0.746 \\
\text { AbsCorr }=\text { MULTI-SCAN }\end{array}$} \\
\hline \multicolumn{3}{|c|}{ Data completeness $=1.000 \quad$ Theta $(\max )=24.997$} \\
\hline \multicolumn{3}{|c|}{$R($ reflections $)=0.0522(1367)$} \\
\hline \multicolumn{3}{|c|}{$S=1.011 \quad$ Npar $=182$} \\
\hline
\end{tabular}

The following ALERTS were generated. Each ALERT has the format test-name_ALERT_alert-type_alert-level.

Click on the hyperlinks for more details of the test.

Alert level C

PLAT906 ALERT 3 C Large K Value in the Analysis of Variance ...... 20.364 Check PLAT906_ALERT_ 3 C L Large K Value in the Analysis of Variance ...... 2.985 Check PLAT978 ALERT 2 C Number C-C Bonds with Positive Residual Density. 0 Info

0 ALERT level $A=$ Most likely a serious problem - resolve or explain

0 ALERT level $B=A$ potentially serious problem, consider carefully

3 ALERT level $\mathrm{C}=$ Check. Ensure it is not caused by an omission or oversight

$\theta$ ALERT level $G=$ General information/check it is not something unexpected

0 ALERT type $1 \mathrm{CIF}$ construction/syntax error, inconsistent or missing data

1 ALERT type 2 Indicator that the structure model may be wrong or deficient

2 ALERT type 3 Indicator that the structure quality may be low

0 ALERT type 4 Improvement, methodology, query or suggestion

0 ALERT type 5 Informative message, check 


\section{Large Scale Dearomative Difluorination}

It was found that upon increasing the concentration of the reaction beyond $1 \mathrm{mmol}$, the reaction immediately turned black and only decomposition of the starting material was observed. For this reason, we added NFSI via a syringe pump, and this allowed us to increase the concentration significantly. The reaction mixture was filtered through a silica plug to remove the polar biproducts of NFSI, then the product was purified by recrystallization or column chromatography.

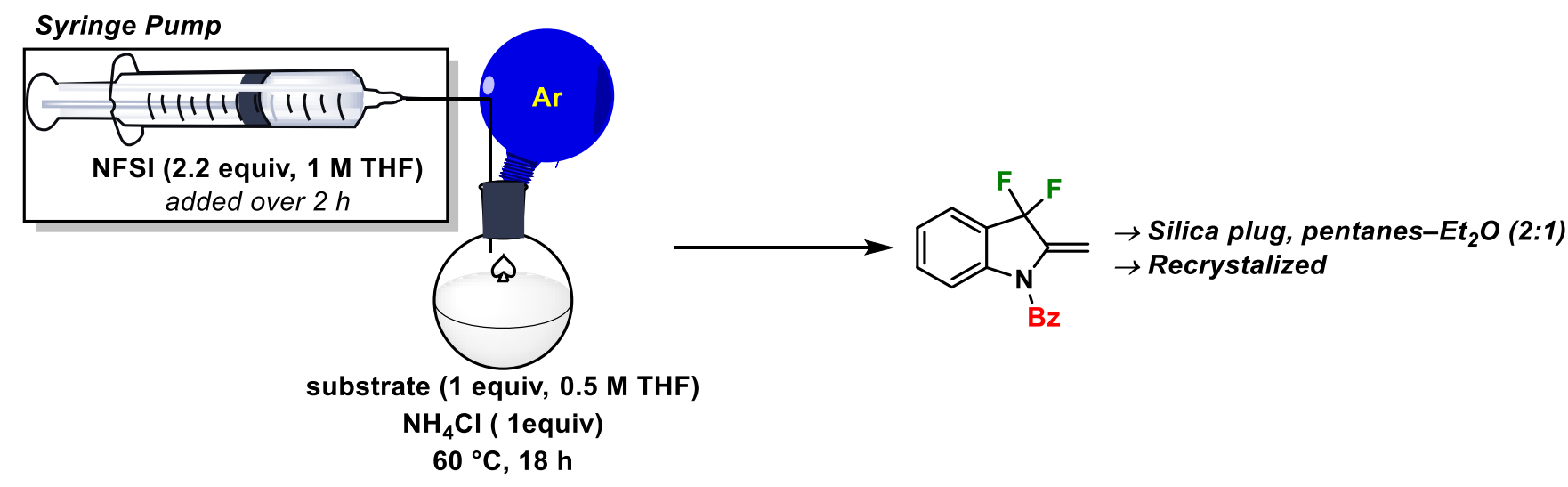

(3,3-difluoro-2-methyleneindolin-1-yl)(phenyl)methanone (2a) - In a flame dried round bottom flask equipped with an argon balloon was added substrate $1 \mathbf{a}$ (11 or $20 \mathrm{mmol}, 1$ equiv), $\mathrm{NH}_{4} \mathrm{Cl}$ (1 equiv), and THF (0.5 M) and was stirred at $60{ }^{\circ} \mathrm{C}$ in an oil bath. NFSI (2.2 equiv) in THF (1 M) was added using a syringe pump over $2 \mathrm{~h}$ and the reaction was further stirred for $24 \mathrm{~h}$. Upon completion, polar materials were crashed out using pentanes and the suspension was filtered over a silica plug eluting with pentanes- $\mathrm{Et}_{2} \mathrm{O}(2: 1)$.

Recrystallization: The product was recrystalized using pentanes and small amounts of $\mathrm{Et}_{2} \mathrm{O}$ to afford analytically pure $2 \mathbf{a}$ (44\% yield for $11 \mathrm{mmol}$ and $20 \mathrm{mmol} \mathrm{scale}$ ).

Column chromatography: The product was purified by column chromatography using pentanes: $\mathrm{Et}_{2} \mathrm{O}(100: 1$, v:v) to afford $2 \mathrm{a}(56 \%$ yield for $11 \mathrm{mmol} \mathrm{scale})$ 


\section{Derivatization and Related studies}

\subsection{Allylic Substitution with Morpholine Nucleophile $\left(\mathbf{S}_{\mathrm{N}} \mathbf{2}^{\prime}\right)$}

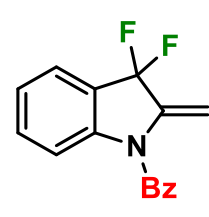

2a

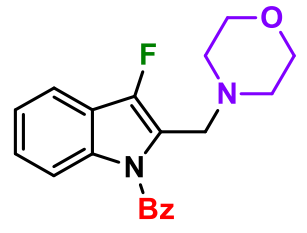

3

(3-fluoro-2-(morpholinomethyl)-1H-indol-1-yl)(phenyl)methanone (3) - In an oven-dried 2-dram vial equipped with an open-top cap with septum and an argon balloon was added substrate $\mathbf{2 a}(0.1 \mathrm{mmol})$, DMA (1 mL), and morpholine (10 equiv) in that order. The balloon was removed, the reaction was sealed tightly, and was heated to $70{ }^{\circ} \mathrm{C}$ in an oil bath for $18 \mathrm{~h}$. The reaction was filtered over silica, concentrated, and columned using a pentanesEtOAc (10:1, v:v) eluting phase to isolated 3 as a white solid $(28.8 \mathrm{mg}, 0.851 \mathrm{mmol}, 85 \%) .{ }^{1} \mathbf{H} \mathbf{~ N M R}(400 \mathrm{MHz}$, $\left.\mathrm{CDCl}_{3}\right) \delta 7.77-7.69(\mathrm{~m}, 2 \mathrm{H}), 7.65-7.54(\mathrm{~m}, 2 \mathrm{H}), 7.54-7.41(\mathrm{~m}, 3 \mathrm{H}), 7.29-7.19(\mathrm{~m}, 2 \mathrm{H}), 3.54-3.43(\mathrm{~m}, 6 \mathrm{H})$, $2.15-2.06(\mathrm{~m}, 4 \mathrm{H}) .{ }^{13} \mathbf{C}$ NMR $\left(100 \mathrm{MHz}, \mathrm{CDCl}_{3}\right) \delta 169.7,147.8(\mathrm{~d}, J=251.8 \mathrm{~Hz}), 135.7,134.2(\mathrm{~d}, J=5.3 \mathrm{~Hz})$, $132.8,129.8,128.6,125.3,122.9,119.0(\mathrm{~d}, J=18.3 \mathrm{~Hz}), 118.8(\mathrm{~d}, J=22.4 \mathrm{~Hz}), 116.9(\mathrm{~d}, J=2.6 \mathrm{~Hz}), 114.3(\mathrm{~d}, J$ $=1.8 \mathrm{~Hz}), 67.0,52.7,50.6(\mathrm{~d}, J=2.7 \mathrm{~Hz}) .{ }^{19} \mathbf{F}$ NMR $\left(377 \mathrm{MHz}, \mathrm{CDCl}_{3}\right) \delta-168.89 . \mathbf{I R}\left(\right.$ thin film, $\left.\mathrm{cm}^{-1}\right) 3058,2962$, 2854, 2814, 1694, 1454, 1411, 1343, 1320, 1116, 747. HRMS (DART, M+H) Calculated for $\mathrm{C}_{20} \mathrm{H}_{20} \mathrm{~N}_{2} \mathrm{O}_{2} \mathrm{~F}$ 339.1503 , found 339.1507 .

\subsection{DMDO Epoxidation}

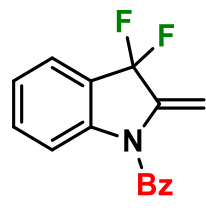

2a

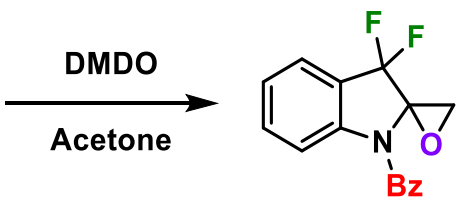

4

(3,3-difluorospiro[indoline-2,2'-oxiran]-1-yl)(phenyl)methanone (4) - DMDO was synthesized according the literature procedure. ${ }^{3} 3.3 \mathrm{~mL}$ of DMDO in acetone $(0.066 \mathrm{M}, 1.1$ equiv) was added to $2 \mathrm{a}$ ( $54.25 \mathrm{mg}, 0.2 \mathrm{mmol}, 1$ equiv) at $0{ }^{\circ} \mathrm{C}$. The reaction was gradually warmed to r.t. and stirred for $20 \mathrm{~h}$. The clear solution was concentrated under reduced pressure to give $\mathbf{4}$ as a slightly yellow oil $(57.4,0.200 \mathrm{mmol},>99 \%) .{ }^{1} \mathbf{H} \mathbf{~ N M R}\left(400 \mathrm{MHz}, \mathrm{CDCl}_{3}\right) \delta$ $7.70-7.53(\mathrm{~m}, 4 \mathrm{H}), 7.53-7.43(\mathrm{~m}, 2 \mathrm{H}), 7.38(\mathrm{tq}, J=7.5,1.2 \mathrm{~Hz}, 1 \mathrm{H}), 7.24(\mathrm{dd}, J=8.0,7.1 \mathrm{~Hz}, 1 \mathrm{H}), 7.13-7.02$ $(\mathrm{m}, 1 \mathrm{H}), 3.75(\mathrm{ddd}, J=4.6,2.7,2.0 \mathrm{~Hz}, 1 \mathrm{H}), 3.31(\mathrm{dd}, J=4.5,0.5 \mathrm{~Hz}, 1 \mathrm{H}) .{ }^{13} \mathbf{C} \mathbf{N M R}\left(125 \mathrm{MHz}, \mathrm{CDCl}_{3}\right) \delta 169.1$, $143.8(\mathrm{dd}, J=6.7,5.4 \mathrm{~Hz}), 134.8,132.8,132.1,128.6,128.3,124.6(\mathrm{t}, J=1.8 \mathrm{~Hz}), 123.7,121.3(\mathrm{dd}, J=25.2,24.7$ $\mathrm{Hz}), 119.4(\mathrm{dd}, J=249.9,243.5 \mathrm{~Hz}), 117.2,76.1(\mathrm{dd}, J=41.6,25.3 \mathrm{~Hz}), 48.2(\mathrm{~d}, J=4.2 \mathrm{~Hz}) .{ }^{19} \mathbf{F ~ N M R}(377 \mathrm{MHz}$, $\left.\mathrm{CDCl}_{3}\right) \delta-93.82(\mathrm{~d}, J=259.7 \mathrm{~Hz}, 1 \mathrm{~F}),-109.25(\mathrm{~d}, J=259.7 \mathrm{~Hz}, 1 \mathrm{~F}) . \mathbf{I R}$ (thin film, $\left.\mathrm{cm}^{-1}\right)$ 3060, 3005, 2920, 1972 , 1674, 1615, 1601, 1473, 1406, 1342, 1294, 1195, 1158, 1140, 1116, 1076, 949, 760, 699. HRMS (DART, M+H) Calculated for $\mathrm{C}_{16} \mathrm{H}_{12} \mathrm{NO}_{2} \mathrm{~F}_{2} 288.0831$, found 288.0830. 


\subsection{Pd-Catalyzed Hydro-Defluorination}

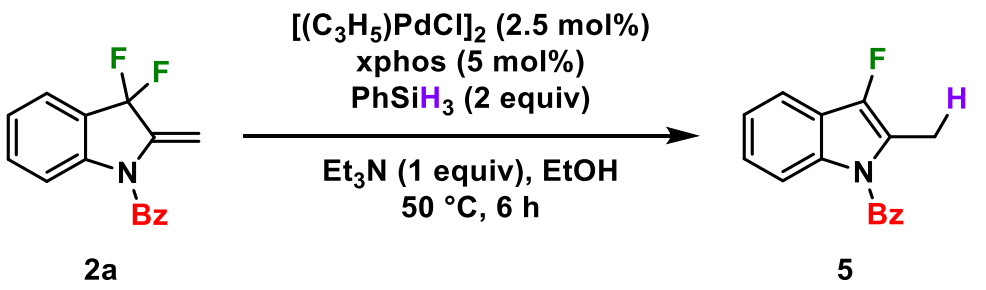

(3-fluoro-2-methyl-1H-indol-1-yl)(phenyl)methanone (5) - In an oven-dried 2-dram vial equipped with an opentop cap with septum and an argon balloon was added substrate $\mathbf{2 a}(0.1 \mathrm{mmol}),\left[\left(\mathrm{C}_{3} \mathrm{H}_{5}\right) \mathrm{PdCl}\right]_{2}(2.5 \mathrm{~mol} \%)$, and xphos ( $5 \mathrm{~mol} \%)$. The vial was purged for 10 minutes and thereafter, $\mathrm{EtOH}(1 \mathrm{~mL}), \mathrm{PhSiH}_{3}$ (2 equiv), and $\mathrm{NEt}_{3}(1$ equiv) were added successively. The balloon was removed, the reaction was sealed tightly and was heated to $50{ }^{\circ} \mathrm{C}$ in an oil bath for $6 \mathrm{~h}$. The reaction was filtered over silica, concentrated, and columned using a pentanes- $\mathrm{Et}_{2} \mathrm{O}(100: 1$, v:v) eluting phase to isolated $\mathbf{5}$ as a white solid $(25.3 \mathrm{mg}, 0.999 \mathrm{mmol},>99 \%) .{ }^{1} \mathbf{H} \mathbf{~ N M R}\left(600 \mathrm{MHz}, \mathrm{CDCl}_{3}\right) \delta 7.73$ $-7.68(\mathrm{~m}, 2 \mathrm{H}), 7.67-7.61(\mathrm{~m}, 1 \mathrm{H}), 7.55-7.47(\mathrm{~m}, 3 \mathrm{H}), 7.21(\mathrm{ddd}, J=7.9,6.8,1.2 \mathrm{~Hz}, 1 \mathrm{H}), 7.15-7.07(\mathrm{~m}, 2 \mathrm{H})$, $2.31(\mathrm{~d}, J=2.5 \mathrm{~Hz}, 3 \mathrm{H}) .{ }^{13} \mathbf{C}$ NMR $\left(125 \mathrm{MHz}, \mathrm{CDCl}_{3}\right) \delta 169.6,147.0(\mathrm{~d}, J=248.3 \mathrm{~Hz}), 135.7,133.5(\mathrm{~d}, J=5.2$ $\mathrm{Hz}), 133.0,129.7,129.0,124.3,123.1,120.4(\mathrm{~d}, J=18.6 \mathrm{~Hz}), 119.6(\mathrm{~d}, J=25.2 \mathrm{~Hz}), 116.3(\mathrm{~d}, J=2.6 \mathrm{~Hz}), 114.8$ $(\mathrm{d}, J=1.9 \mathrm{~Hz}), 11.1(\mathrm{~d}, J=1.5 \mathrm{~Hz}) .{ }^{19} \mathbf{F}$ NMR $\left(564 \mathrm{MHz}, \mathrm{CDCl}_{3}\right) \delta-171.22$. IR (thin film, $\left.\mathrm{cm}^{-1}\right) 3058,2964,2927$, 2854, 1687, 1645, 1600, 1456, 1347, 1316, 743. HRMS (DART, M+H) Calculated for $\mathrm{C}_{16} \mathrm{H}_{13} \mathrm{NOF} 254.0976$, found 254.0977.

\subsection{Pd-Catalyzed Double Amination}<smiles>C=C1N(C(=O)c2ccccc2)c2ccccc2C1(F)F</smiles>

2a
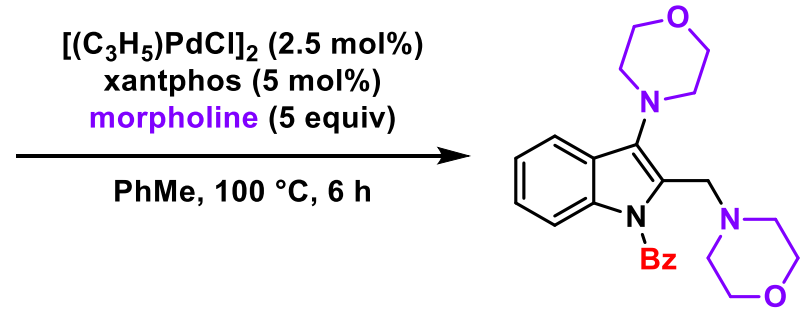

6

(3-morpholino-2-(morpholinomethyl)-1H-indol-1-yl)(phenyl)methanone (6) - In an oven-dried 2-dram vial equipped with an open-top cap with septum and an argon balloon was added substrate $\mathbf{2 a}(0.1 \mathrm{mmol}),\left[\left(\mathrm{C}_{3} \mathrm{H}_{5}\right) \mathrm{PdCl}\right]_{2}$ $(2.5 \mathrm{~mol} \%)$, and Xantphos $(5 \mathrm{~mol} \%)$. The vial was purged for 10 minutes and thereafter, $\mathrm{PhMe}(1 \mathrm{~mL})$, and morpholine (5 equiv) were added successively. The balloon was removed, the reaction was sealed tightly, and it was heated to $100^{\circ} \mathrm{C}$ in an oil bath for $6 \mathrm{~h}$. The reaction was filtered over silica, concentrated, and columned using a pentanes-EtOAc (10:1, v:v) eluting phase to isolated $\mathbf{6}$ as a white solid $(32.3 \mathrm{mg}, 0.0796 \mathrm{mmol}, 80 \%) .{ }^{\mathbf{1}} \mathbf{H}$ NMR $\left(400 \mathrm{MHz} \mathrm{CDCl}_{3}\right) \delta 7.78-7.70(\mathrm{~m}, 3 \mathrm{H}), 7.63-7.54(\mathrm{~m}, 1 \mathrm{H}), 7.51-7.43(\mathrm{~m}, 3 \mathrm{H}), 7.21-7.13(\mathrm{~m}, 2 \mathrm{H}), 3.89-$ $3.83(\mathrm{~m}, 4 \mathrm{H}), 3.51(\mathrm{~s}, 2 \mathrm{H}), 3.46(\mathrm{t}, J=4.7 \mathrm{~Hz}, 4 \mathrm{H}), 3.28-3.21(\mathrm{~m}, 4 \mathrm{H}), 2.09-2.02(\mathrm{~m}, 4 \mathrm{H}) .{ }^{13} \mathbf{C ~ N M R}(125 \mathrm{MHz}$, $\left.\mathrm{CDCl}_{3}\right) \delta 170.0,136.3,135.9,133.2,132.7,130.8,129.9,128.5,125.7,123.9,122.0,119.8,114.1,68.0,67.0,52.9$, 52.8, 51.9. IR (thin film, $\mathrm{cm}^{-1}$ ) 2958, 2852, 1688, 1454, 1344, 1327, 1261, 1115, 748. HRMS (DART, M+H) Calculated for $\mathrm{C}_{24} \mathrm{H}_{28} \mathrm{~N}_{3} \mathrm{O}_{3} 406.2125$, found 406.2127 . 


\subsection{Pd-Catalyzed Double sulfonylation}
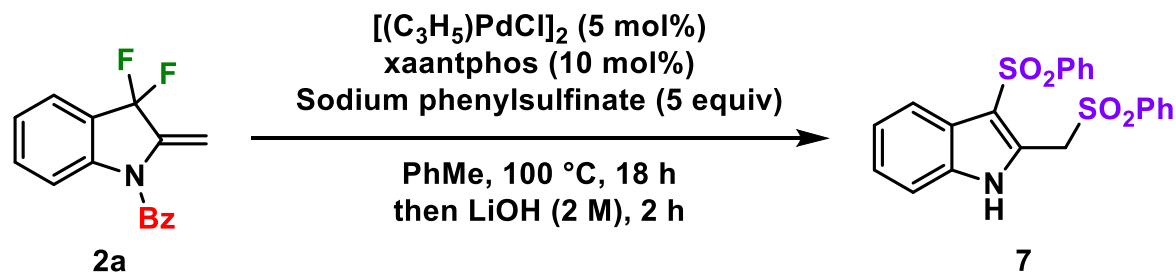

3-(phenylsulfonyl)-2-((phenylsulfonyl)methyl)-1H-indole (7) - In an oven-dried 2-dram vial equipped with an open-top cap with septum and an argon balloon was added substrate $\mathbf{2 a}(0.2 \mathrm{mmol}),\left[\left(\mathrm{C}_{3} \mathrm{H}_{5}\right) \mathrm{PdCl}\right]_{2}(5 \mathrm{~mol} \%)$, Xantphos (10 mol\%), and sodium benzene sulfinate (5 equiv). The vial was purged for 10 minutes and thereafter, PhMe ( $1 \mathrm{~mL})$ was added. The balloon was removed, the reaction was sealed tightly, and it was heated to $100{ }^{\circ} \mathrm{C}$ in an oil bath for $18 \mathrm{~h}$. The reaction was filtered over silica, concentrated, and columned using a pentanes-EtOAc (5:1 to 2:1, v:v) eluting phase to isolate 7 as a white solid (40.3 mg, $0.0979 \mathrm{mmol}, 49 \%) .{ }^{1} \mathbf{H} \mathbf{~ N M R}\left(400 \mathrm{MHz}, \mathrm{CDCl}_{3}\right)$ $\delta 9.72(\mathrm{~s}, 1 \mathrm{H}), 7.86-7.77(\mathrm{~m}, 3 \mathrm{H}), 7.77-7.70(\mathrm{~m}, 2 \mathrm{H}), 7.70-7.61(\mathrm{~m}, 1 \mathrm{H}), 7.59-7.41(\mathrm{~m}, 4 \mathrm{H}), 7.41-7.33(\mathrm{~m}$, 2H), $7.33-7.28(\mathrm{~m}, 1 \mathrm{H}), 7.22(\mathrm{ddd}, \mathrm{J}=8.2,7.1,1.1 \mathrm{~Hz}, 1 \mathrm{H}), 5.25(\mathrm{~s}, 2 \mathrm{H}) .{ }^{13} \mathbf{C} \mathbf{N M R}\left(125 \mathrm{MHz}, \mathrm{CDCl}_{3}\right) \delta 142.9$, 137.6, 135.5, 134.7, 132.9, 129.6, 129.6, 129.2, 128.5, 126.5, 125.0, 124.3, 123.0, 120.2, 115.4, 112.1, 52.7. IR (thin film, $\mathrm{cm}^{-1}$ ) 3317, 3069, 3011, 2918, 1534, 1447, 1426, 1302, 1131, 908, 733. HRMS (DART, M+H) Calculated for $\mathrm{C}_{21} \mathrm{H}_{21} \mathrm{~N}_{2} \mathrm{O}_{4} \mathrm{~S}_{2} 429.0937$, found 429.0933 .

\subsection{Pd-Catalyzed Allylic Phosphorylation}

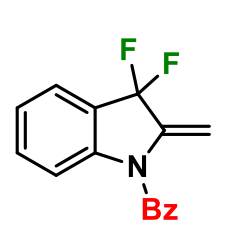

2a

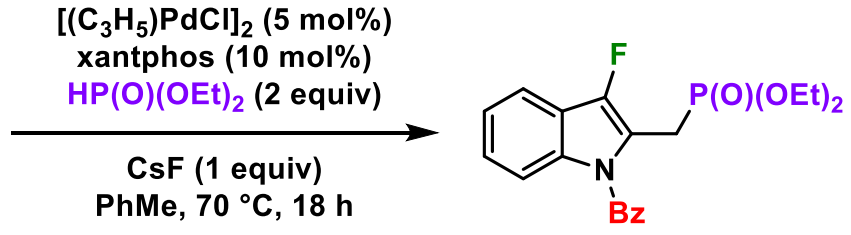

8

diethyl ((1-benzoyl-3-fluoro-1H-indol-2-yl)methyl)phosphonate (8) - In an oven-dried 2-dram vial equipped with an open-top cap with septum and an argon balloon was added substrate $\mathbf{2 a}(0.1 \mathrm{mmol}),\left[\left(\mathrm{C}_{3} \mathrm{H}_{5}\right) \mathrm{PdCl}\right]_{2}(2.5$ mol\%), and Xantphos (5 mol\%), and $\mathrm{CsF}$ (1 equiv). The vial was purged for 10 minutes and thereafter, PhMe (1 $\mathrm{mL}$ ), and diethyl phosphite ( 2 equiv) were added successively. The balloon was removed, the reaction was sealed tightly, and it was heated to $70{ }^{\circ} \mathrm{C}$ in an oil bath for $18 \mathrm{~h}$. The reaction was filtered over silica, concentrated, and columned using a pentanes-EtOAc (3:1 to 2:1, v:v) eluting phase to isolate $\mathbf{8}$ as a white solid (26.1 $\mathrm{mg}, 0.0670$ mmol, 67\%). ${ }^{1} \mathbf{H}$ NMR $\left(400 \mathrm{MHz}, \mathrm{CDCl}_{3}\right) \delta 7.83-7.76(\mathrm{~m}, 2 \mathrm{H}), 7.69-7.60(\mathrm{~m}, 1 \mathrm{H}), 7.52$ (dddd, J = 13.8, 7.7, 6.9, 1.4 Hz, 3H), $7.17(\mathrm{ddd}, \mathrm{J}=8.0,7.2,0.9 \mathrm{~Hz}, 1 \mathrm{H}), 7.01(\mathrm{ddt}, \mathrm{J}=8.5,7.2,1.3 \mathrm{~Hz}, 1 \mathrm{H}), 6.63(\mathrm{dd}, \mathrm{J}=8.6,2.2 \mathrm{~Hz}$, $1 \mathrm{H}), 4.01(\mathrm{dqd}, \mathrm{J}=8.3,7.1,2.5 \mathrm{~Hz}, 4 \mathrm{H}), 3.74(\mathrm{dd}, \mathrm{J}=21.0,1.5 \mathrm{~Hz}, 2 \mathrm{H}), 1.17(\mathrm{t}, \mathrm{J}=7.1 \mathrm{~Hz}, 6 \mathrm{H}) .{ }^{13} \mathbf{C}$ NMR $(125$ $\left.\mathrm{MHz}, \mathrm{CDCl}_{3}\right) \delta 169.7(\mathrm{~d}, J=1.1 \mathrm{~Hz}), 147.8(\mathrm{dd}, J=252.0,11.2 \mathrm{~Hz}), 135.1,134.0(\mathrm{dd}, J=5.4,1.9 \mathrm{~Hz}), 133.3$, $130.3,128.9,124.5(\mathrm{~d}, J=2.1 \mathrm{~Hz}), 122.7(\mathrm{~d}, J=1.6 \mathrm{~Hz}), 119.3(\mathrm{dd}, J=17.9,4.0 \mathrm{~Hz}), 116.7(\mathrm{dd}, J=2.3 \mathrm{~Hz}), 114.6$ $(\mathrm{dd}, J=24.5,14.7 \mathrm{~Hz}), 114.2(\mathrm{dd}, J=1.8 \mathrm{~Hz}), 62.4(\mathrm{~d}, J=6.5 \mathrm{~Hz}), 22.2(\mathrm{dd}, J=141.0,2.2 \mathrm{~Hz}), 16.3(\mathrm{~d}, J=5.9$ $\mathrm{Hz}) .{ }^{19} \mathbf{F}$ NMR $\left(377 \mathrm{MHz}, \mathrm{CDCl}_{3}\right) \delta-168.38(\mathrm{~d}, \mathrm{~J}=12.1 \mathrm{~Hz}) .{ }^{31} \mathbf{P} \mathbf{N M R}\left(162 \mathrm{MHz}, \mathrm{CDCl}_{3}\right) \delta 23.03(\mathrm{~d}, \mathrm{~J}=11.9$ Hz). IR (thin film, $\mathrm{cm}^{-1}$ ) 3062, 2983, 2931, 3909, 1692, 1638, 1453, 1349, 1019, 853. HRMS (DART, M+H) Calculated for $\mathrm{C}_{20} \mathrm{H}_{22} \mathrm{FNO}_{4} \mathrm{P} 390.1265$, found 390.1266 . 


\subsection{Pd-Catalyzed Double Alkylation}

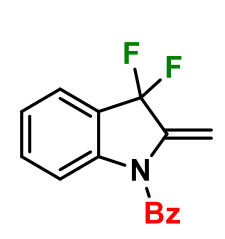

$2 a$

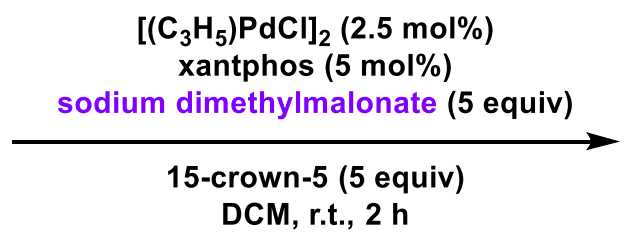

DCM, r.t., 2 h

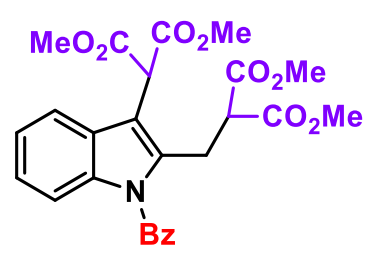

9

dimethyl 2-(1-benzoyl-2-(3-methoxy-2-(methoxycarbonyl)-3-oxopropyl)-1H-indol-3-yl)malonate (9) - In an oven-dried 2-dram vial equipped with an open-top cap with septum and an argon balloon was added substrate 2a (0.1 mmol), $\left[\left(\mathrm{C}_{3} \mathrm{H}_{5}\right) \mathrm{PdCl}\right]_{2}(2.5 \mathrm{~mol} \%)$, and Xantphos $(5 \mathrm{~mol} \%)$, sodium dimethylmalonate (5 equiv), and 15crown-5 (5 equiv). The vial was purged for 10 minutes and thereafter, DCM $(1 \mathrm{~mL})$ was added. The balloon was removed, the reaction was sealed tightly, and it was stirred at r.t. for $2 \mathrm{~h}$. The reaction was filtered over silica, concentrated, and columned using a pentanes-EtOAc (10:1 to 5:1, v:v) eluting phase to isolate 9 as a white solid (46.4 mg, 0.0936 mmol, 94\%). ${ }^{1} \mathbf{H}$ NMR (500 MHz, $\left.\mathrm{CDCl}_{3}\right) \delta 7.75-7.69$ (m, 2H), 7.66 (ddt, $J=8.8,7.3,1.3 \mathrm{~Hz}$, $1 \mathrm{H}), 7.61$ (ddd, $J=8.0,1.3,0.7 \mathrm{~Hz}, 1 \mathrm{H}), 7.54-7.46(\mathrm{~m}, 2 \mathrm{H}), 7.12(\mathrm{ddd}, J=8.0,7.2,1.0 \mathrm{~Hz}, 1 \mathrm{H}), 6.94$ (ddd, $J=$ 8.4, 7.2, 1.2 Hz, 1H), $6.48(\mathrm{dt}, J=8.5,0.9 \mathrm{~Hz}, 1 \mathrm{H}), 5.16(\mathrm{~s}, 1 \mathrm{H}), 4.07$ (t, $J=7.6 \mathrm{~Hz}, 1 \mathrm{H}), 3.78(\mathrm{~s}, 7 \mathrm{H}), 3.69-3.64$ $(\mathrm{m}, 8 \mathrm{H}) .{ }^{13} \mathbf{C} \mathbf{N M R}\left(125 \mathrm{MHz}, \mathrm{CDCl}_{3}\right) \delta 170.0,169.2,168.5,136.7,136.0,134.9,133.6,130.1,129.0,128.1,123.6$, 122.8, 121.3, 114.4, 114.2, 52.9, 52.9, 51.6, 49.0, 25.2. IR (thin film, $\mathrm{cm}^{-1}$ ) 3655, 2981, 2973, 2889, 1755, 1737 , 1458, 1311, 1154. HRMS (DART, M+H) Calculated for $\mathrm{C}_{26} \mathrm{H}_{26} \mathrm{NO}_{9} 496.1602$, found 496.1597.

\subsection{Rhodium-Catalyzed Arylation}

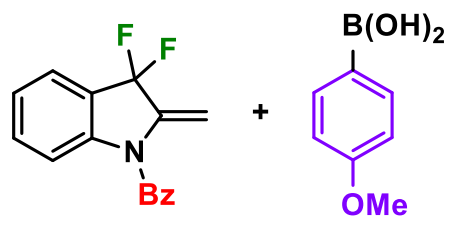

2.5 equiv

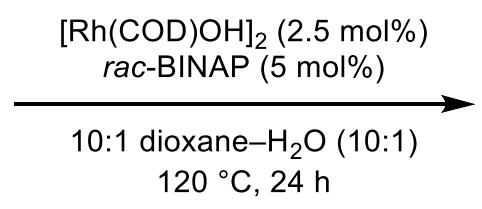

(3-fluoro-2-(4-methoxybenzyl)-1H-indol-1-yl)(phenyl)methanone (12) - In an oven-dried 2-dram vial equipped with an open-top cap with septum and an argon balloon was added substrate $\mathbf{2 a}(0.2 \mathrm{mmol})$, aryl boronic acid (2.5 equiv), $[\mathrm{Rh}(\mathrm{COD}) \mathrm{OH}]_{2}(2.5 \mathrm{~mol} \%)$, and rac-BINAP $(5 \mathrm{~mol} \%)$. The vial was purged for 10 minutes and thereafter, dioxane $(1 \mathrm{~mL})$ and distilled $\mathrm{H}_{2} \mathrm{O}(0.1 \mathrm{~mL})$ was added. The balloon was removed, the reaction was sealed tightly, and it was stirred at $120^{\circ} \mathrm{C}$ in an oil bath for $24 \mathrm{~h}$. The reaction was cooled to room temperature and quenched with an aqueous solution of saturated $\mathrm{NaHCO}_{3}$. The mixture was diluted with EtOAc, washed once with an aqueous solution of $\mathrm{NaHCO}_{3}$, once with $\mathrm{H}_{2} \mathrm{O}$, and once with brine. The organic layer was dried over magnesium sulfate, filtered, and concentrated under reduced pressure. The crude was purified by flash column chromatography pentanes-EtOAc (9:1, v:v) to obtain product 12 as a yellow film $(40.2 \mathrm{mg}, 0.112 \mathrm{mmol}, 56 \%) .{ }^{1} \mathbf{H} \mathbf{~ N M R}(500 \mathrm{MHz}$, $\left.\mathrm{CDCl}_{3}\right) \delta 7.63-7.57(\mathrm{~m}, 2 \mathrm{H}), 7.55-7.50(\mathrm{~m}, 2 \mathrm{H}), 7.46-7.40(\mathrm{~m}, 2 \mathrm{H}), 7.22(\mathrm{ddd}, J=8.0,7.2,0.8 \mathrm{~Hz}, 1 \mathrm{H}), 7.07$ (ddd, $J=8.5,7.2,1.3 \mathrm{~Hz}, 1 \mathrm{H}), 7.00-6.93(\mathrm{~m}, 2 \mathrm{H}), 6.84(\mathrm{ddt}, J=8.5,2.3,0.8 \mathrm{~Hz}, 1 \mathrm{H}), 6.75-6.71(\mathrm{~m}, 2 \mathrm{H}), 4.24$ $(\mathrm{d}, J=1.9 \mathrm{~Hz}, 2 \mathrm{H}), 3.73(\mathrm{~s}, 3 \mathrm{H}) .{ }^{13} \mathbf{C ~ N M R}\left(125 \mathrm{MHz}, \mathrm{CDCl}_{3}\right) \delta 169.4(\mathrm{~d}, J=1.2 \mathrm{~Hz}), 158.3,147.3(\mathrm{~d}, J=250.0$ $\mathrm{Hz}), 135.3,133.9$ (d, $J=5.4 \mathrm{~Hz}), 133.0,130.3(\mathrm{~d}, J=2.0 \mathrm{~Hz}), 129.8,129.4,128.8,124.5,122.9,122.9(\mathrm{~d}, J=24.0$ $\mathrm{Hz}), 119.9(\mathrm{~d}, J=18.6 \mathrm{~Hz}), 116.7(\mathrm{~d}, J=2.6 \mathrm{~Hz}), 114.5(\mathrm{~d}, J=1.8 \mathrm{~Hz}), 113.9,55.3,29.2(\mathrm{~d}, J=1.8 \mathrm{~Hz}) .{ }^{19} \mathbf{F}$ NMR $\left(377 \mathrm{MHz}, \mathrm{CDCl}_{3}\right) \delta-170.14$. IR (neat) 3063, 2913, 2988, 2843, 1693, 1639, 1607, 1511, 1458, 1355, 1313, 1235 , 
1174, 1110, 1047, 894, 755, 720, 699, 663, 638. HRMS (DART, M+H) Calculated for $\mathrm{C}_{23} \mathrm{H}_{19} \mathrm{NO}_{2} \mathrm{~F} 360.1394$, found 360.1396 .

\subsection{Copper-Catalyzed Borylation}
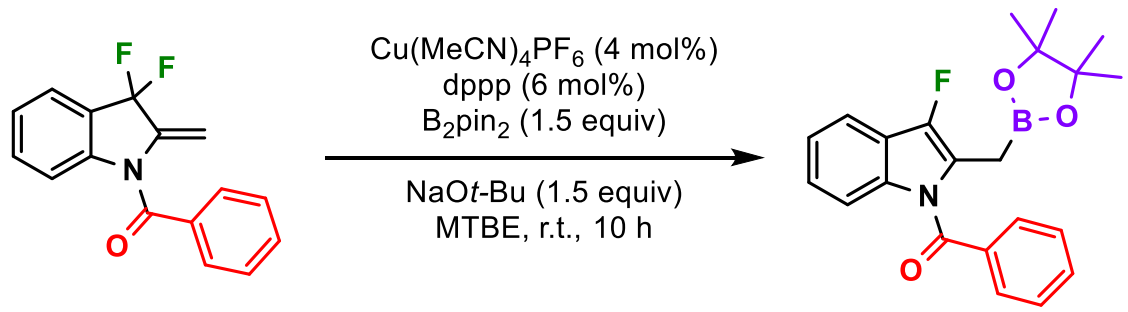

(3-fluoro-2-((4,4,5,5-tetramethyl-1,3,2-dioxaborolan-2-yl)methyl)-1H-indol-1-yl)(phenyl)methanone (13) - In an oven-dried 2-dram vial equipped with an open-top cap with septum and an argon balloon was added Tetrakisacetonitrile copper hexafluorophosphate (4 mol\%), bppp (6 mol\%), and sodium tert-butoxide (1.5 equiv). Anhydrous MTBE (1 mL) was added to the vial and the catalyst solution was stirred for 5 minutes. Bis(pinacolato)diboron (1.5 equiv) was added in $1 \mathrm{~mL}$ of anhydrous MTBE and the suspension was stirred for 5 minutes. Substrate 2a $(0.2 \mathrm{mmol}, 1$ equiv) was dissolved in anhydrous MTBE $(2 \mathrm{~mL})$ and charged to the reaction vial. The reaction was stirred for $10 \mathrm{~h}$ then filtered through a celite pad and concentrated. A crude NMR with TMB as an internal standard suggested the yield is around $89 \%$. The product was purified by flash column chromatography using pentanes-EtOAc (6\% to $10 \%)$ to isolate $\mathbf{1 3}$ as a white solid $(60.6 \mathrm{mg}, 0.160,80 \%) .{ }^{1} \mathbf{H}$ NMR $\left(500 \mathrm{MHz}, \mathrm{CDCl}_{3}\right) \delta 7.79-7.71(\mathrm{~m}, 2 \mathrm{H}), 7.67-7.60(\mathrm{~m}, 1 \mathrm{H}), 7.55-7.45(\mathrm{~m}, 3 \mathrm{H}), 7.18-7.11(\mathrm{~m}, 1 \mathrm{H}), 6.92(\mathrm{ddd}$, $J=8.5,7.2,1.3 \mathrm{~Hz}, 1 \mathrm{H}), 6.47(\mathrm{dd}, J=8.5,2.2 \mathrm{~Hz}, 1 \mathrm{H}), 2.66(\mathrm{~d}, J=1.9 \mathrm{~Hz}, 2 \mathrm{H}), 1.13(\mathrm{~s}, 12 \mathrm{H}) .{ }^{13} \mathbf{C}$ NMR $(125$ $\left.\mathrm{MHz}, \mathrm{CDCl}_{3}\right) \delta 170.0(\mathrm{~d}, J=1.3 \mathrm{~Hz}), 146.2(\mathrm{~d}, J=245.9 \mathrm{~Hz}), 135.7,132.9(\mathrm{~d}, J=5.3 \mathrm{~Hz}), 132.8,130.0,128.7$, 123.2, 122.6, $122.1(\mathrm{~d}, J=25.4 \mathrm{~Hz}), 120.6(\mathrm{~d}, J=18.7 \mathrm{~Hz}), 116.1(\mathrm{~d}, J=2.6 \mathrm{~Hz}), 114.3(\mathrm{~d}, J=1.8 \mathrm{~Hz}), 83.9,24.8$, 9.6. ${ }^{19}$ F NMR $\left(377 \mathrm{MHz}, \mathrm{CDCl}_{3}\right) \delta-172.70$. IR (thin film, $\mathrm{cm}^{-1}$ ) 2936, 1692, 1631, 1448, 1323, 1238, 1204, 1143, 970, 845, 767, 713, 658. HRMS (DART, M+H) Calculated for $\mathrm{C}_{22} \mathrm{H}_{24} \mathrm{BNO}_{3} \mathrm{~F} 380.1834$, found 380.1828 . 


\section{References}

(1) Ramella, V.; He, Z.; Daniliuc, C. G.; Studer, A., Org. Lett. 2015, 17, 664.

(2) Liu, H. H.; Wang, Y.; Deng, G. J.; Yang, L., Advanced Synthesis \& Catalysis 2013, 355, 3369.

(3) Organic Syntheses 2013, 90, 350. 
7 The ${ }^{1} \mathrm{H},{ }^{13} \mathrm{C}$, and ${ }^{19} \mathrm{~F}$ NMR Spectra 
ஸิ

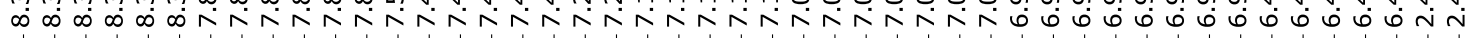
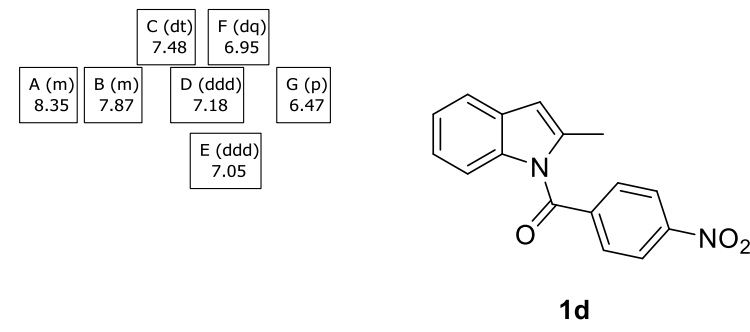

$\mathrm{CDCl}_{3}$ $400 \mathrm{MHz}$

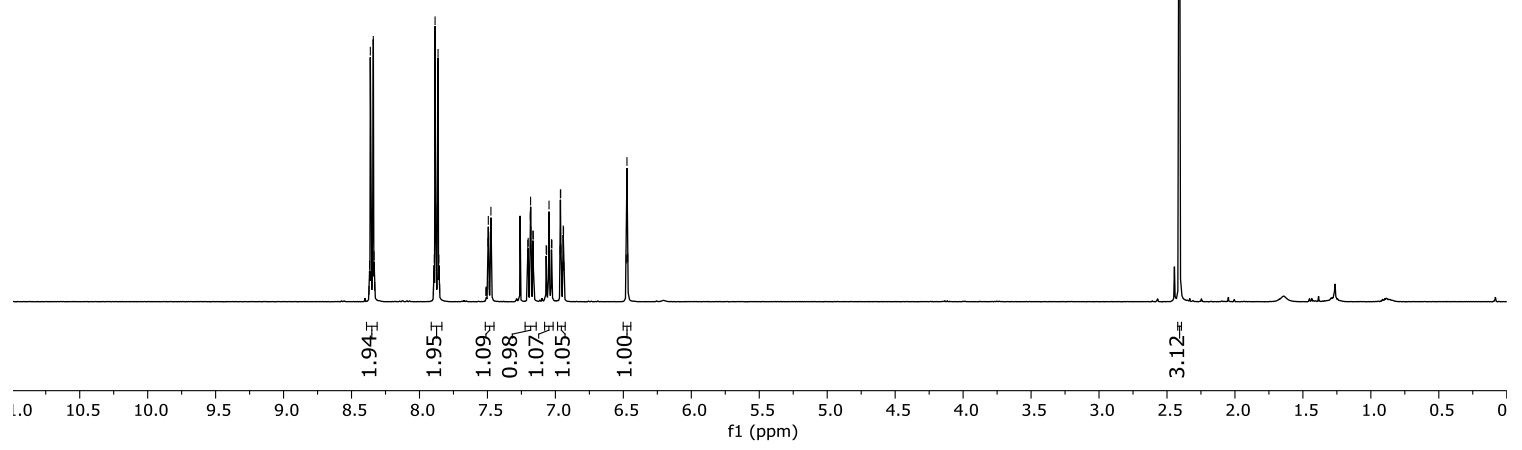

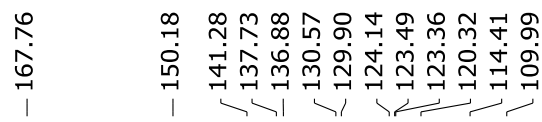

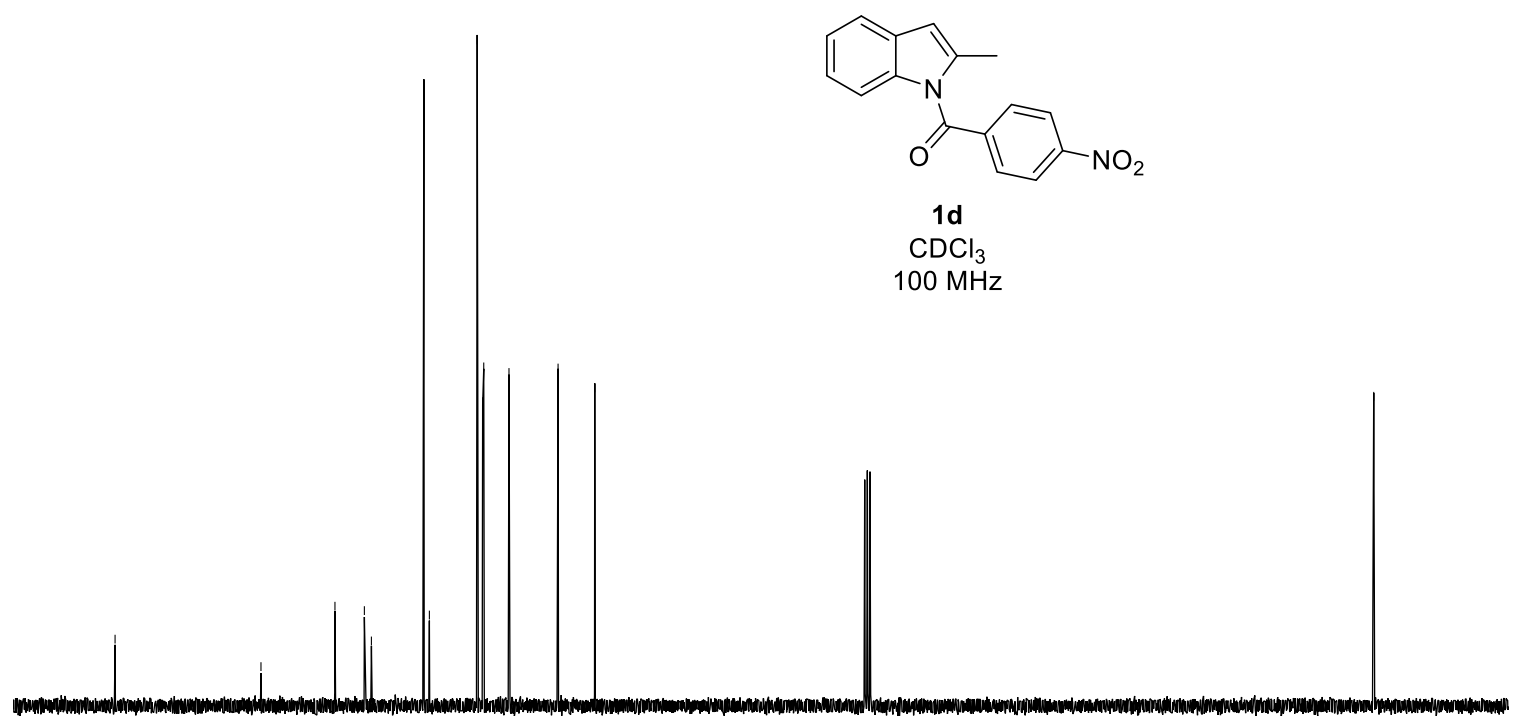

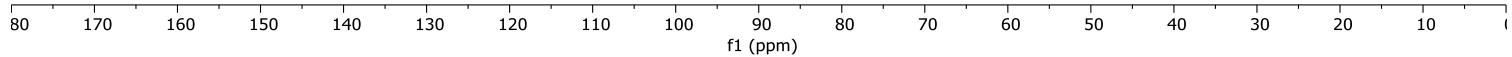




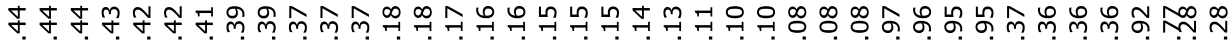
Кง
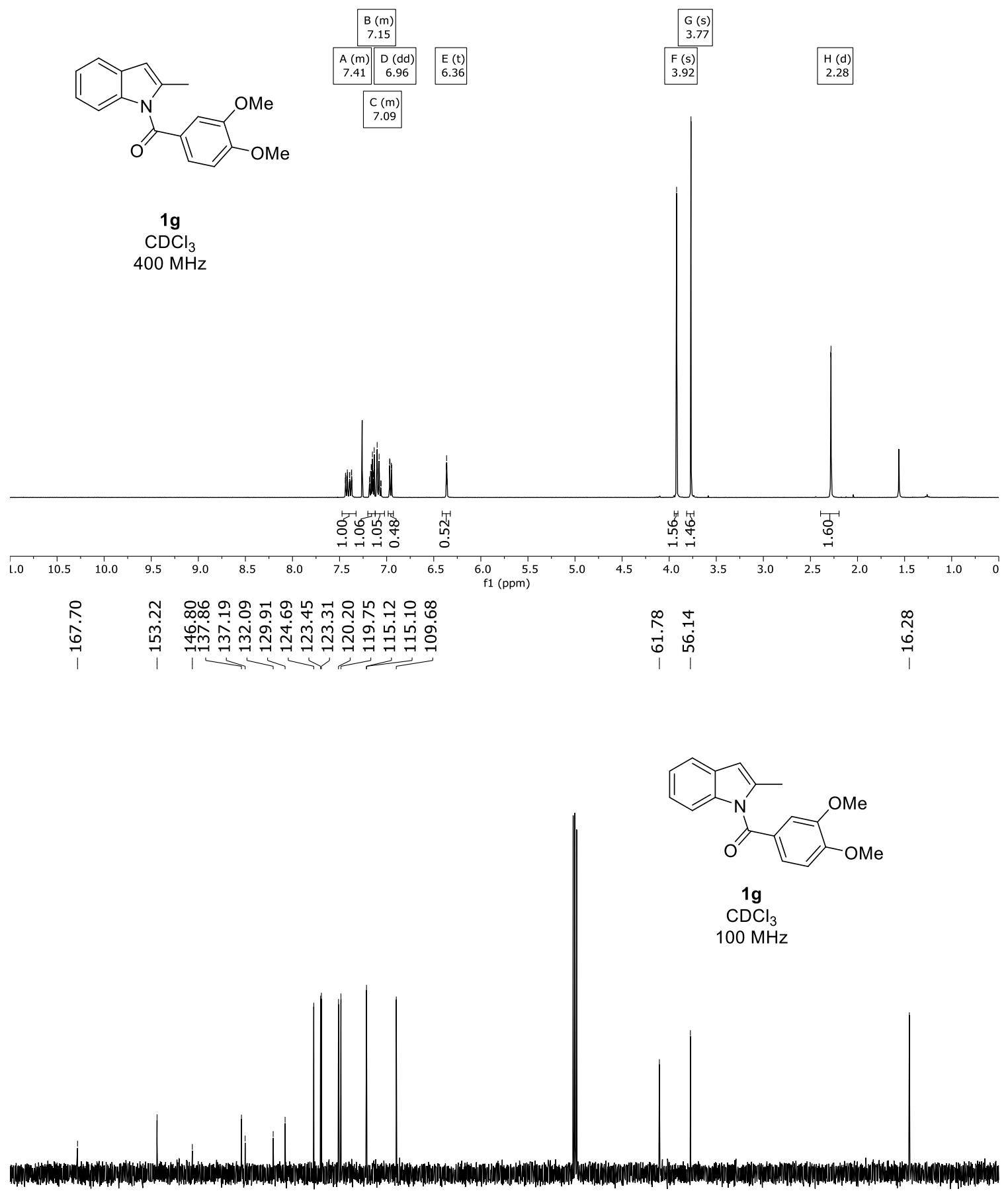

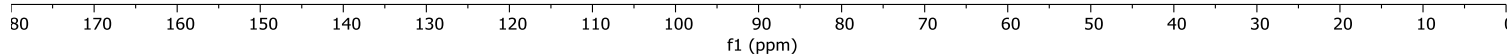




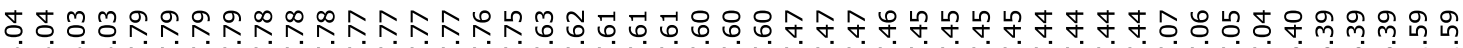

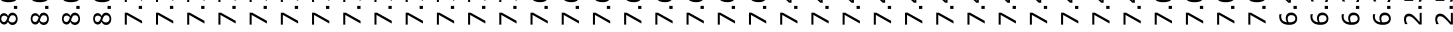

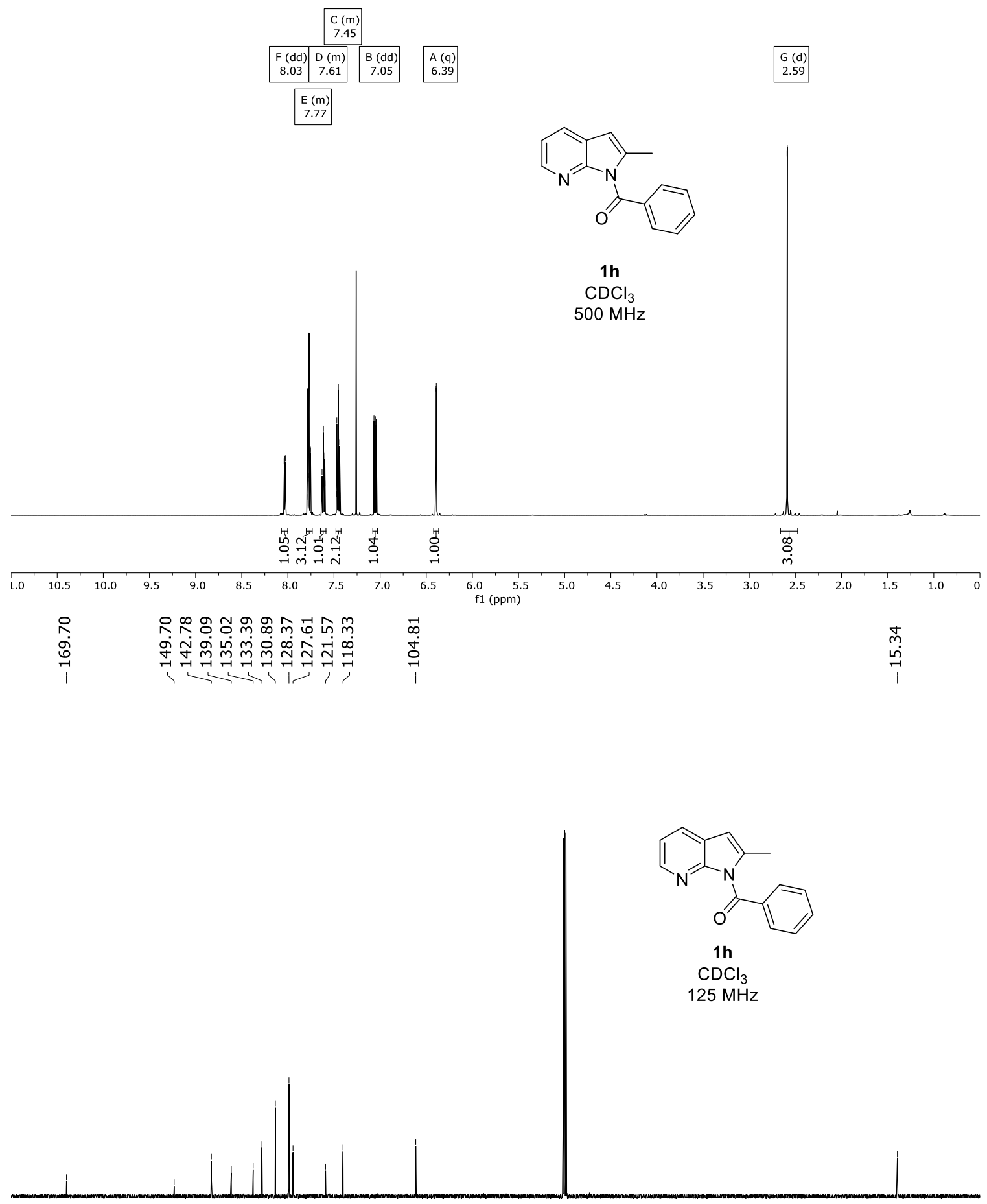

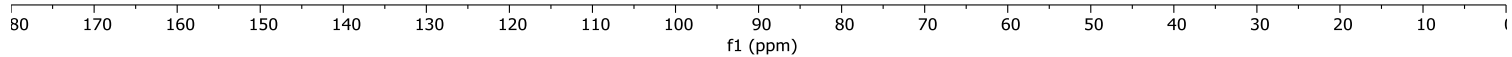




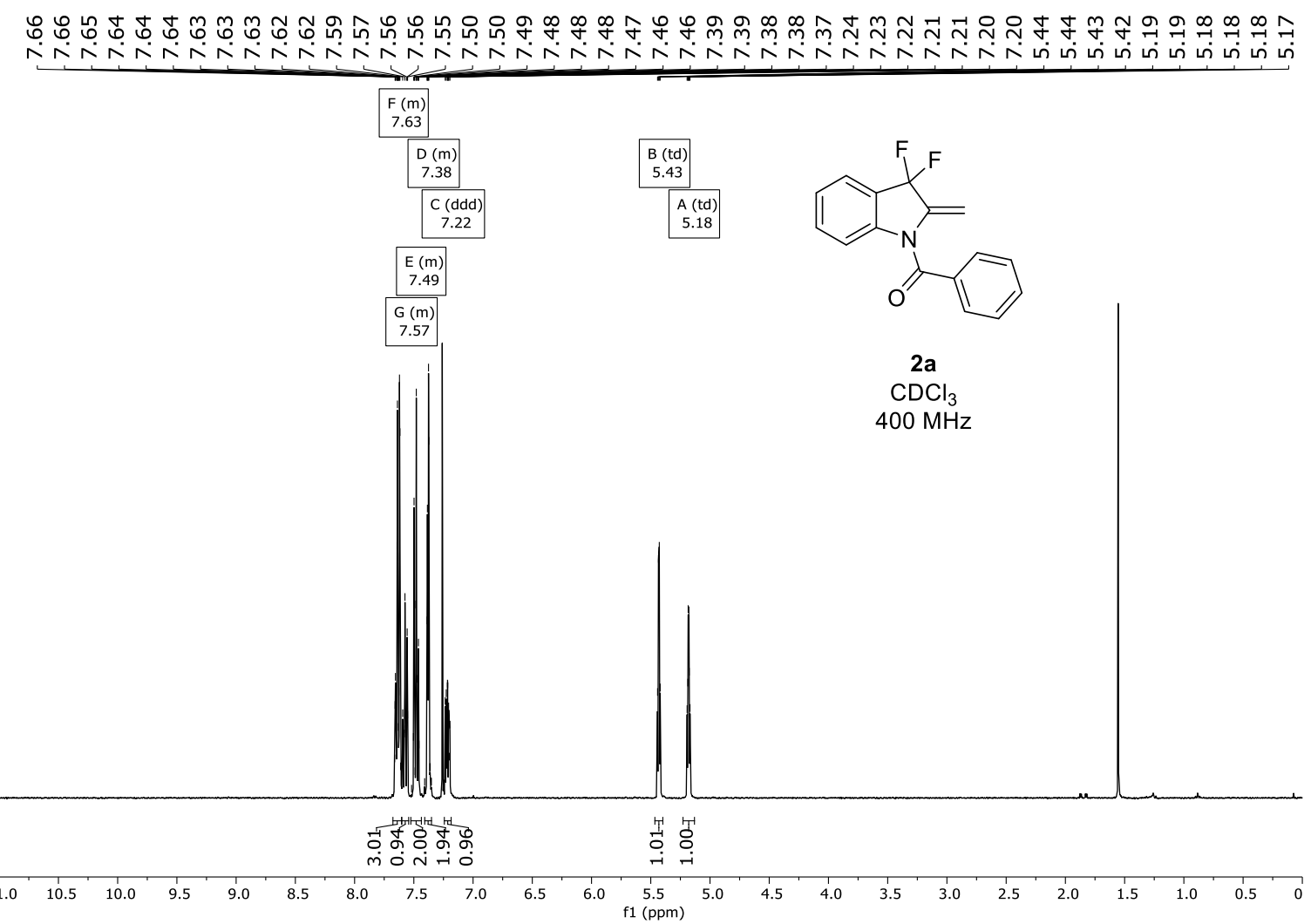

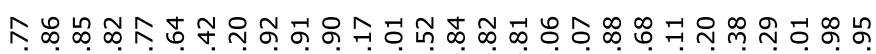

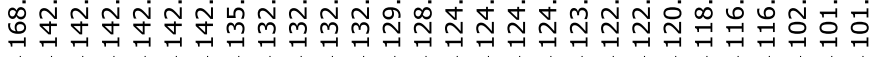

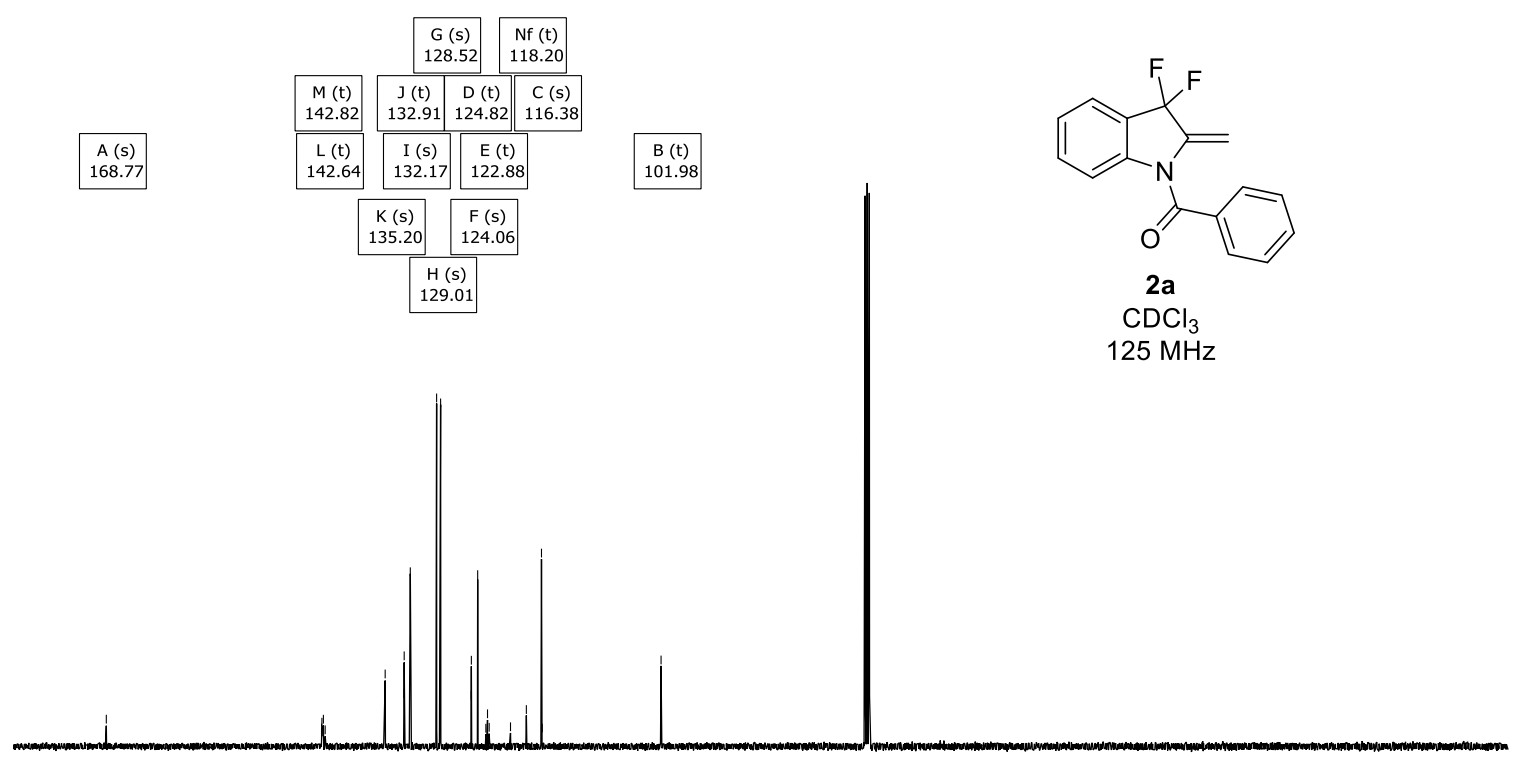

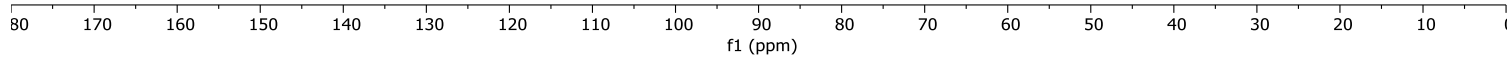




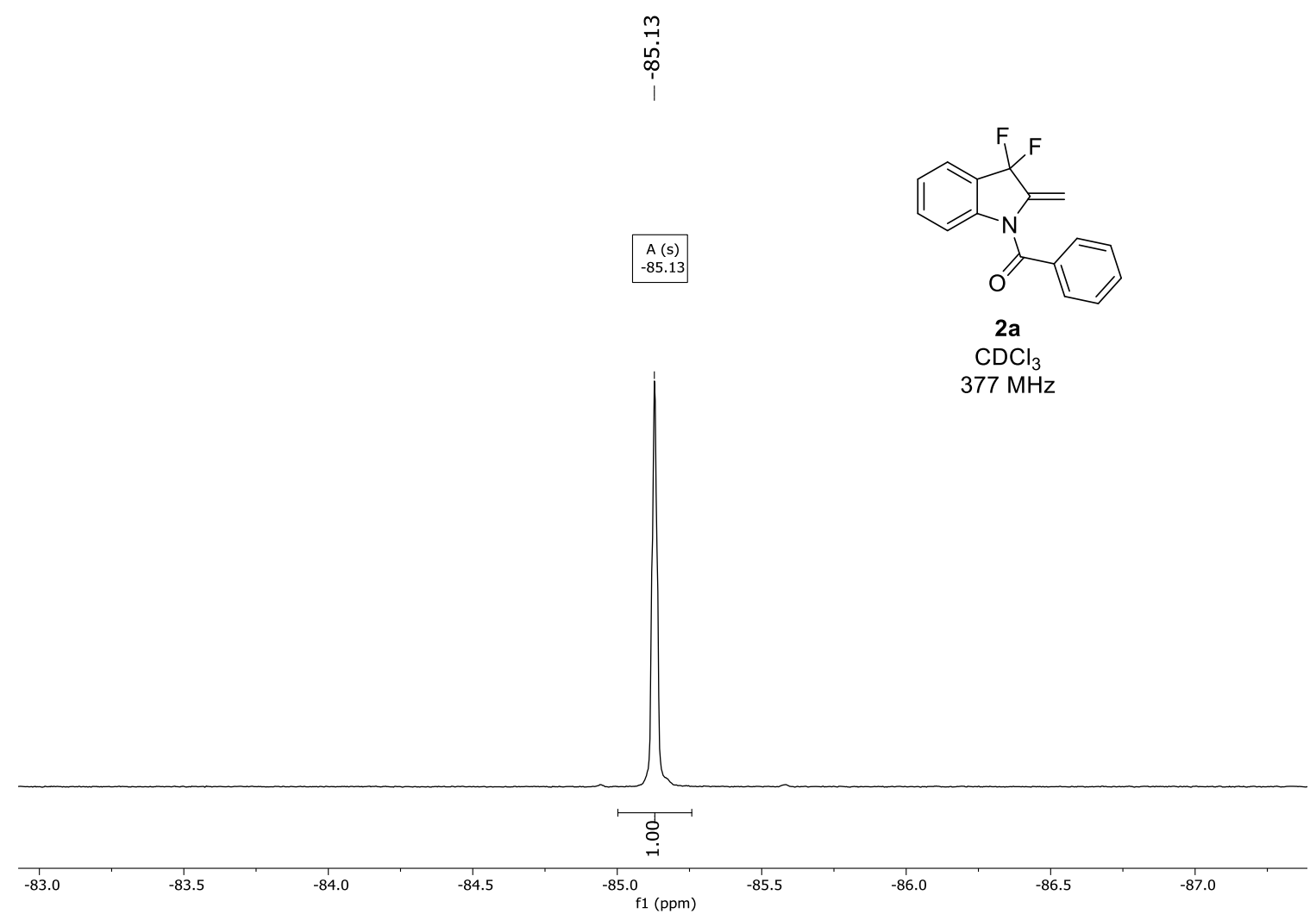




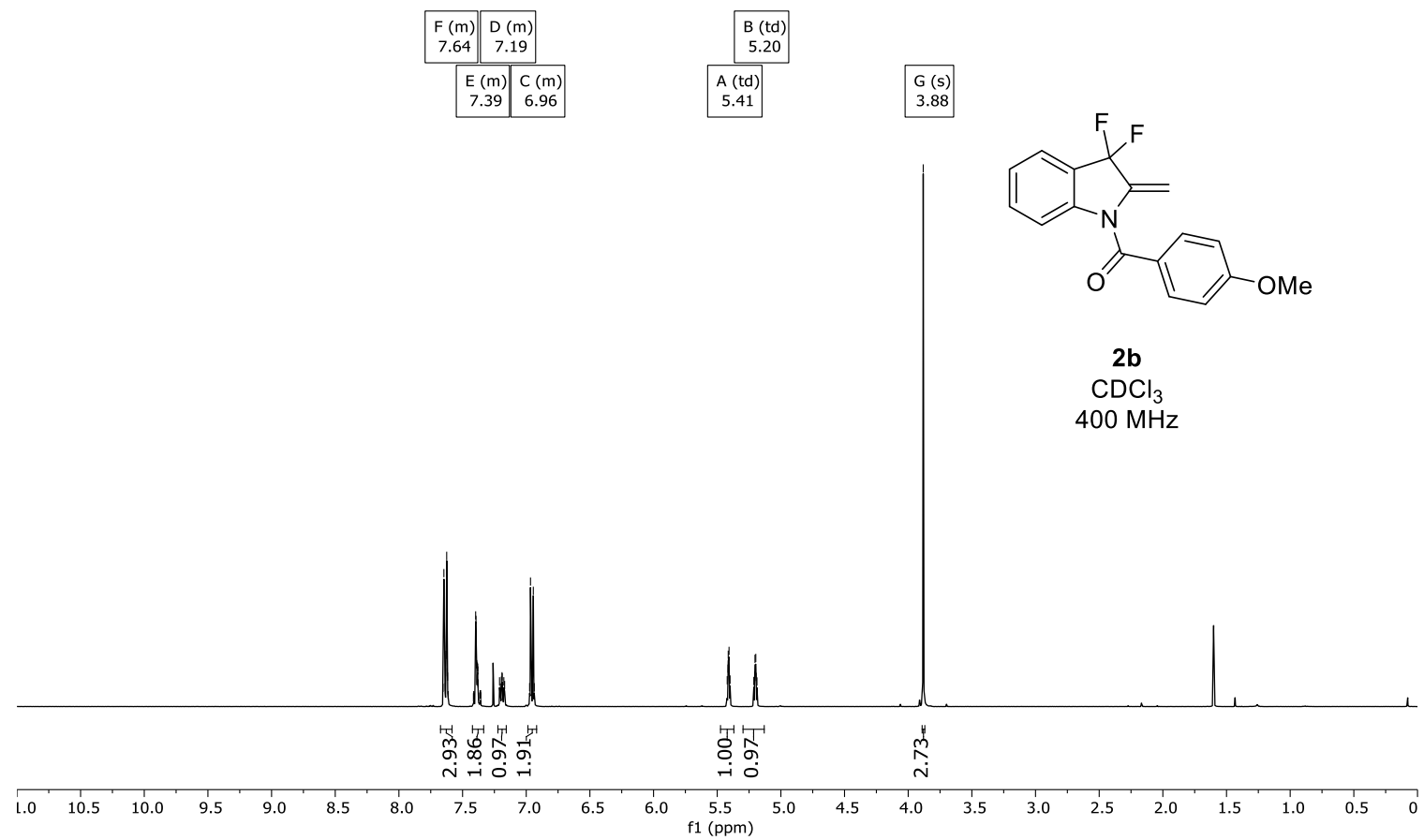

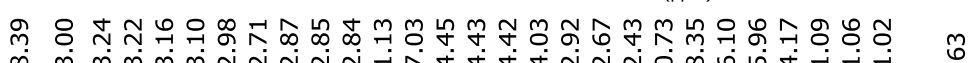

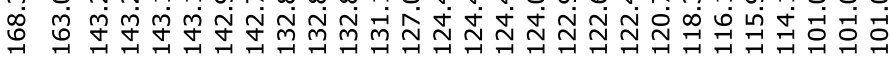

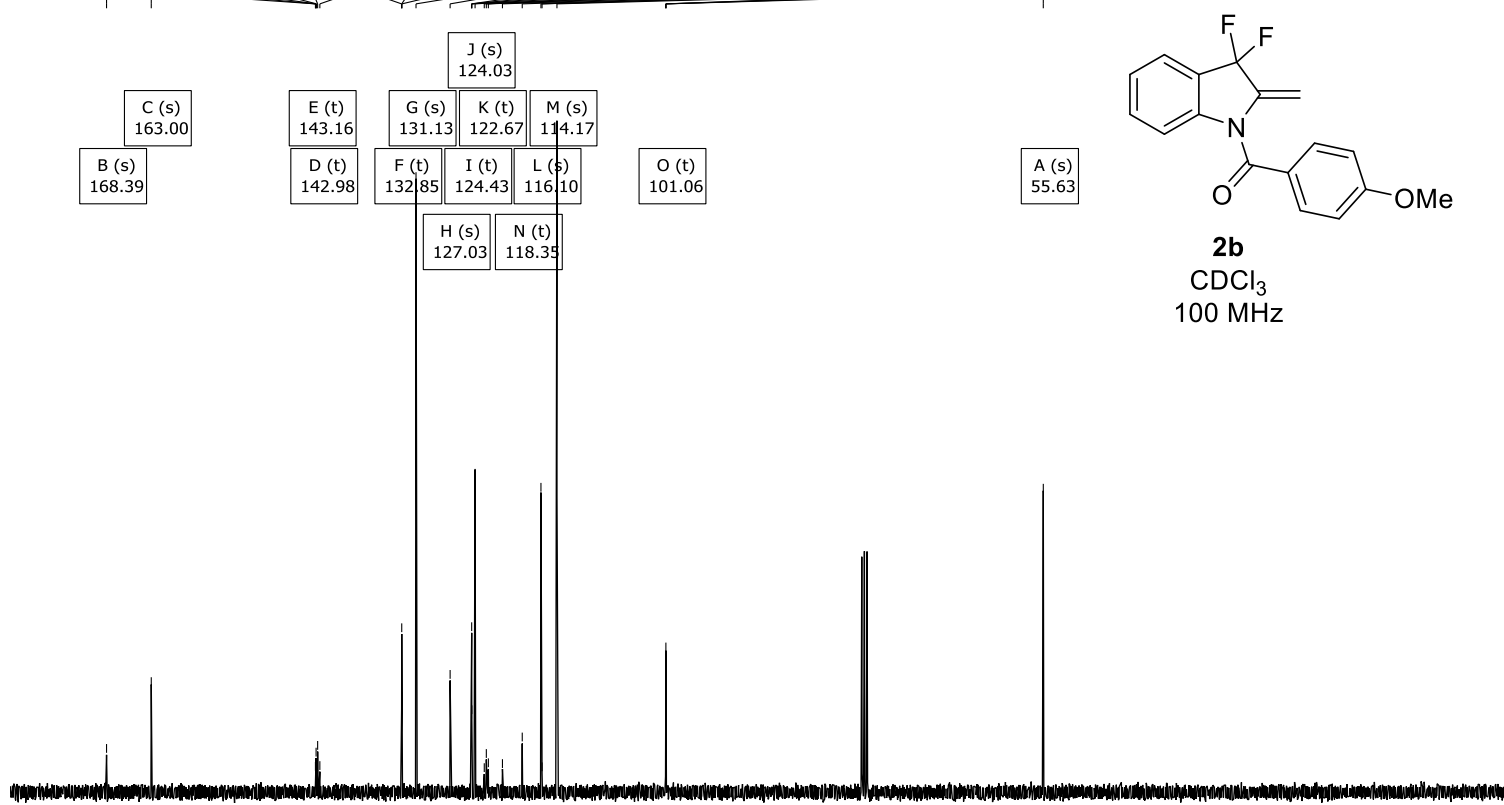

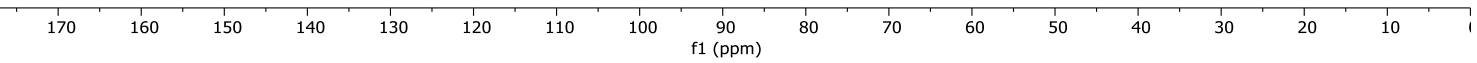




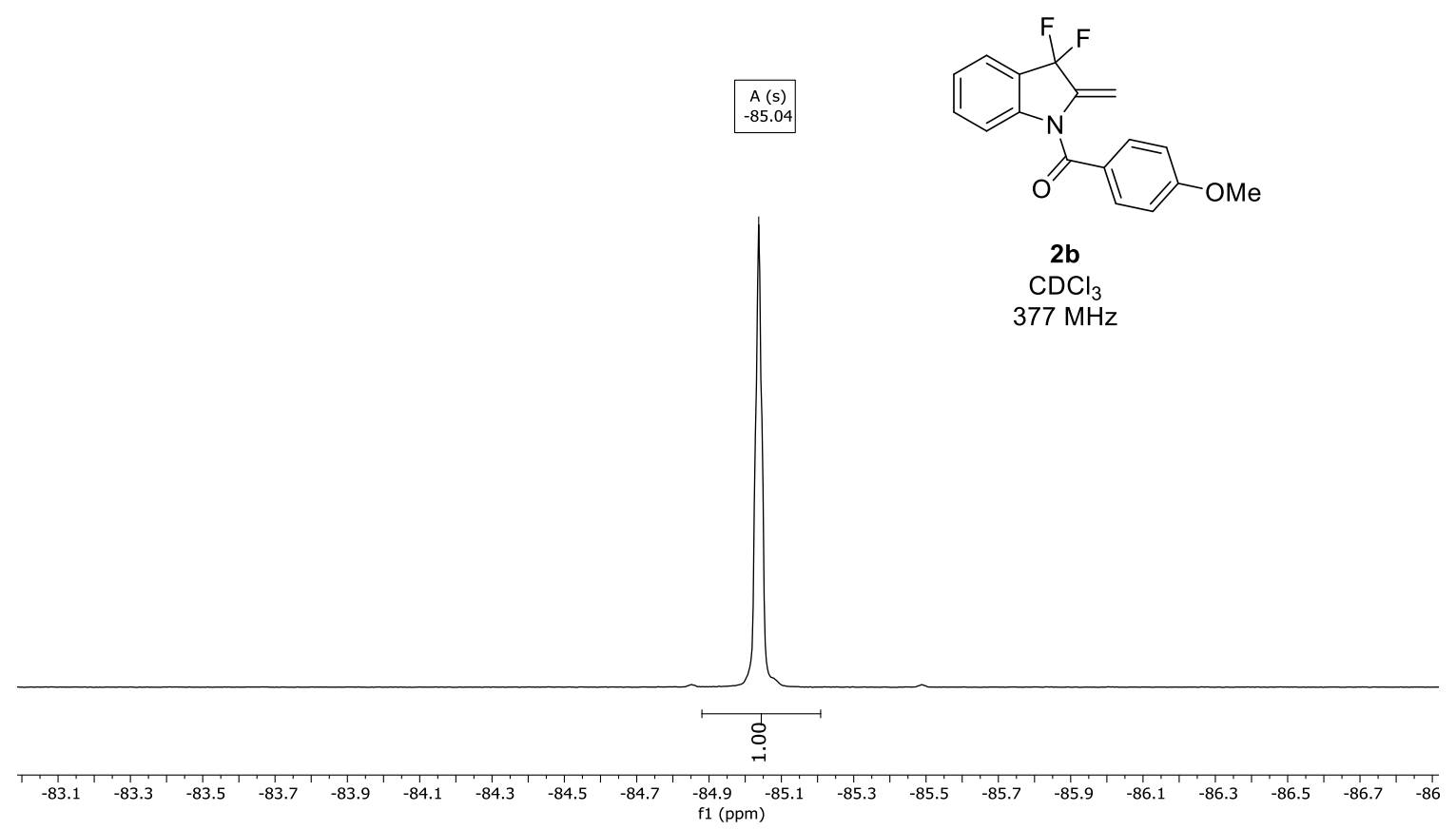




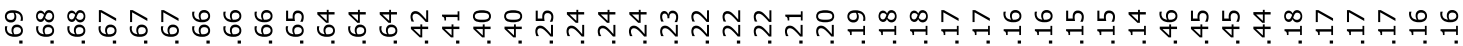

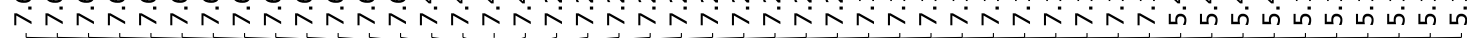
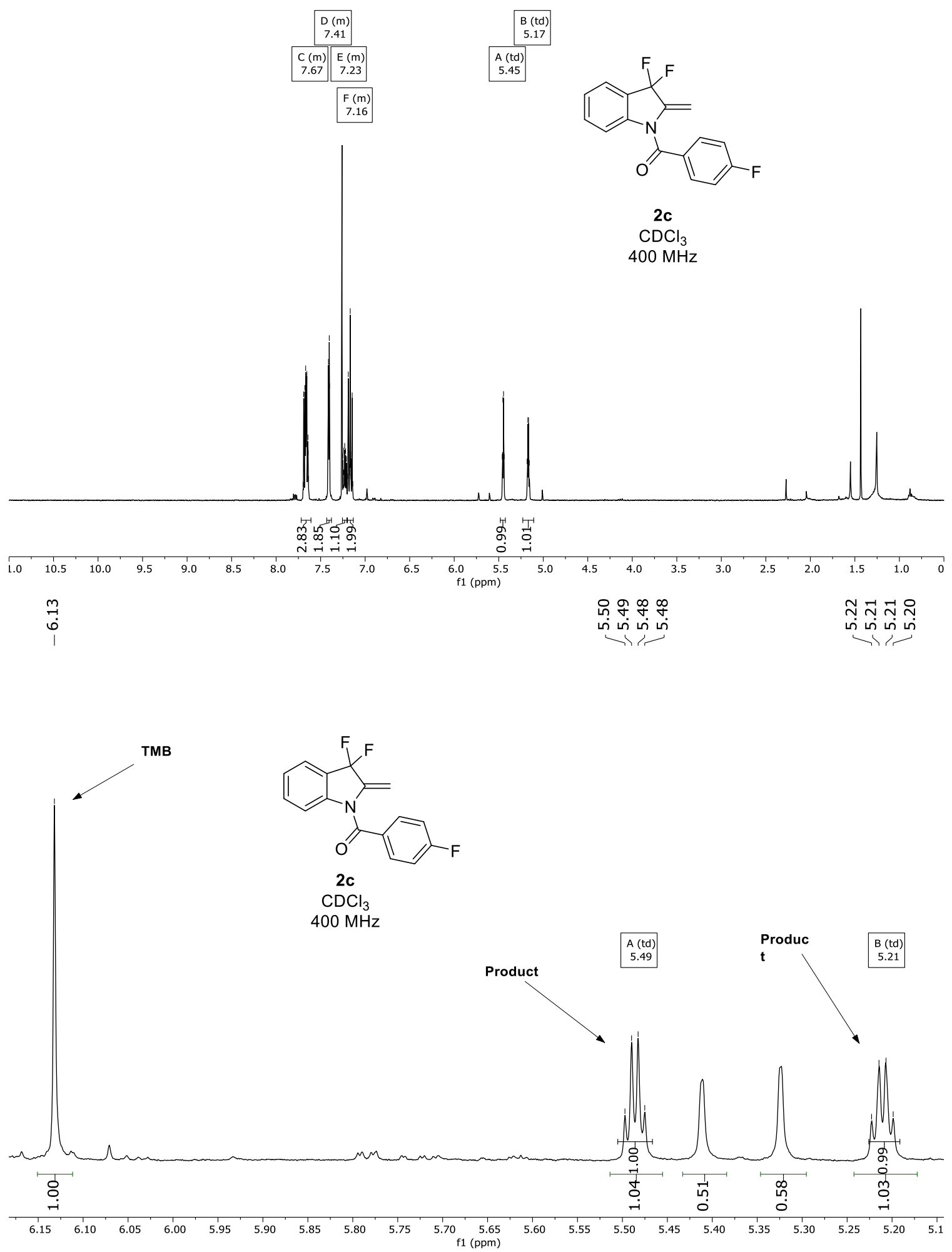

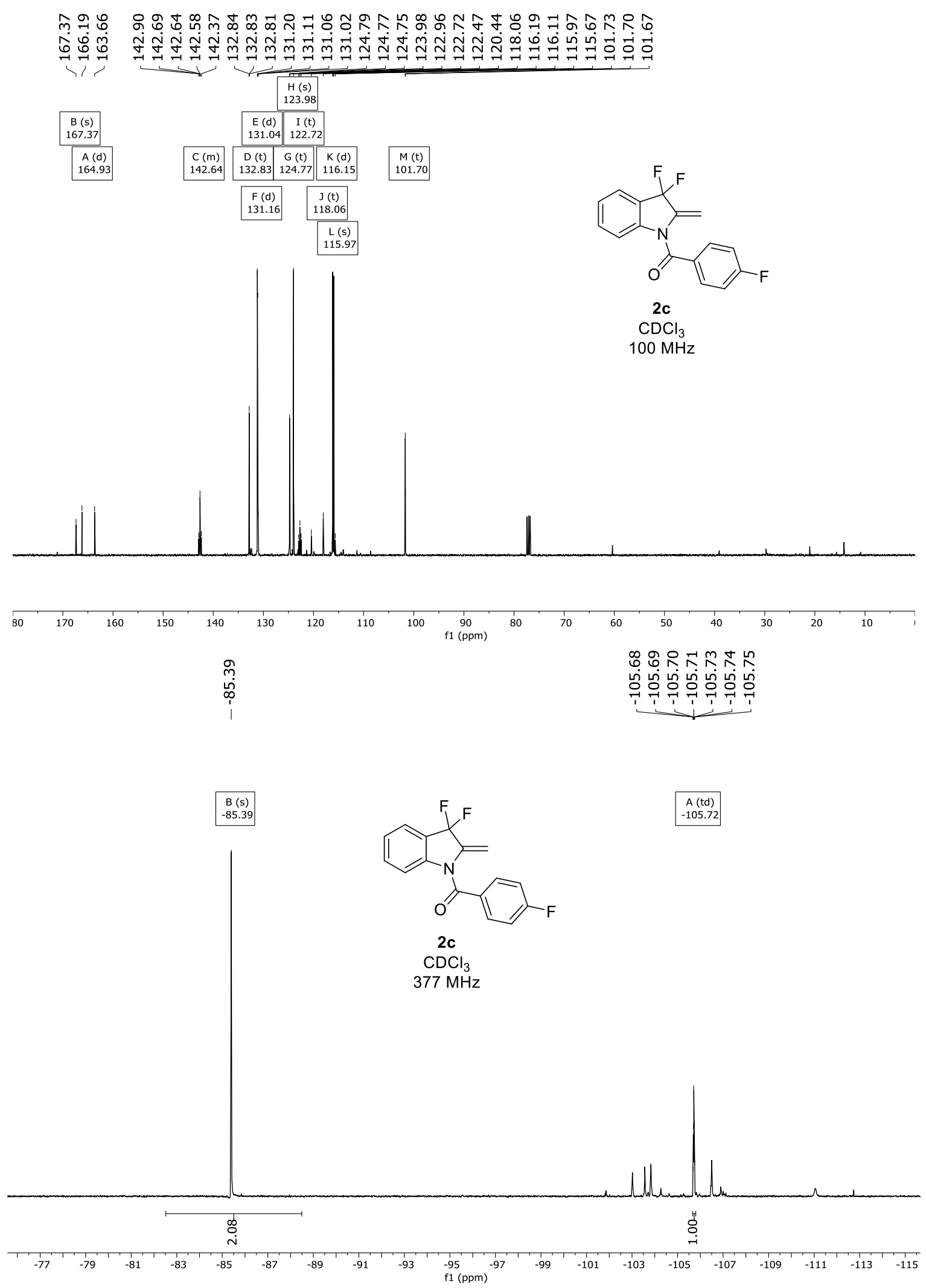
융

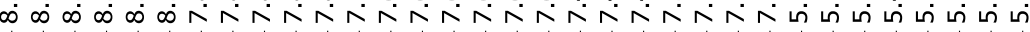

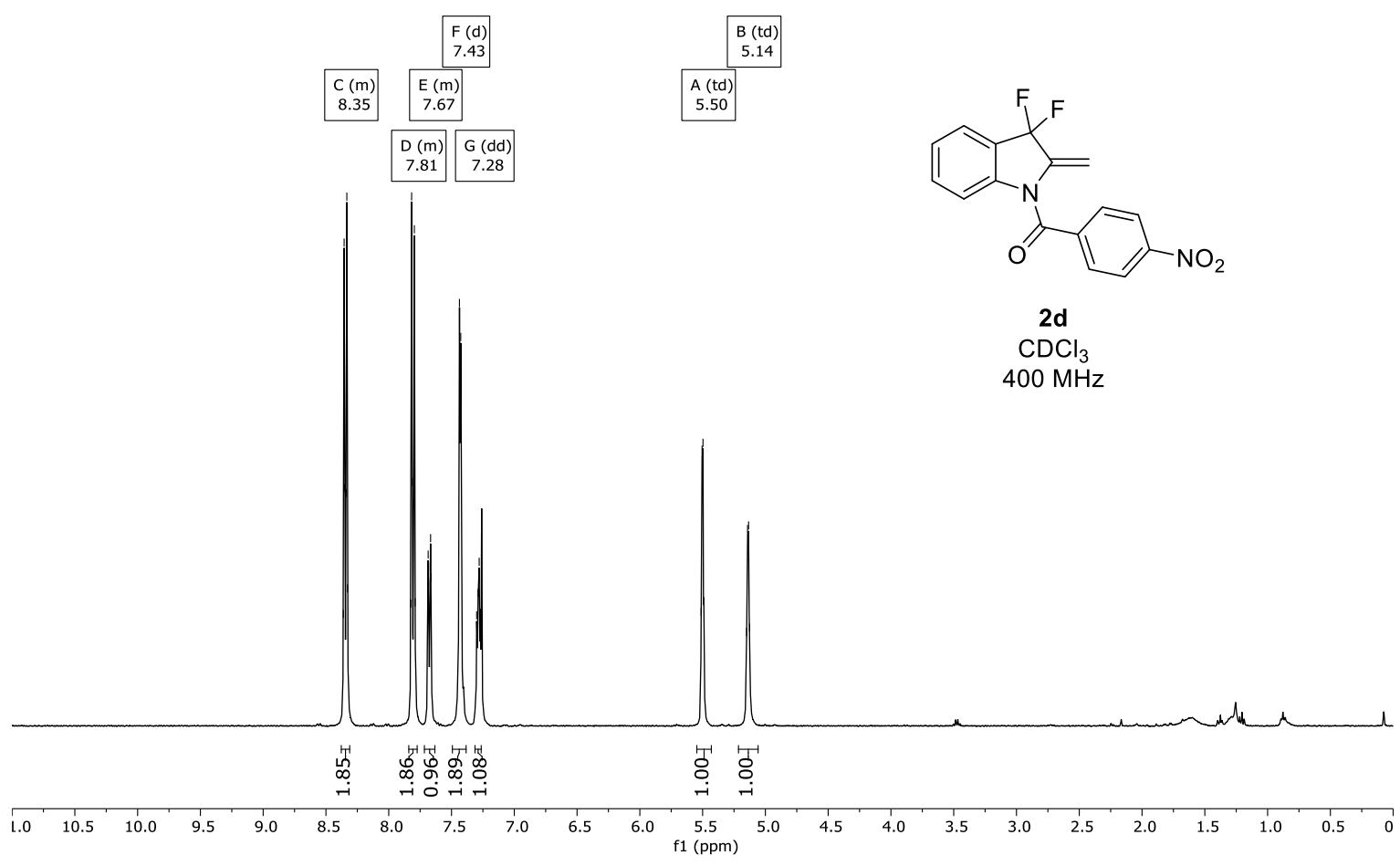

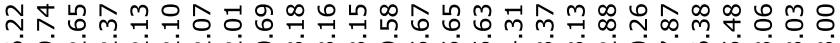

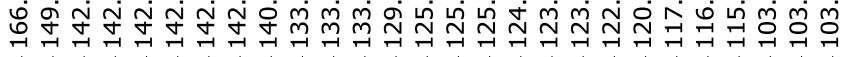

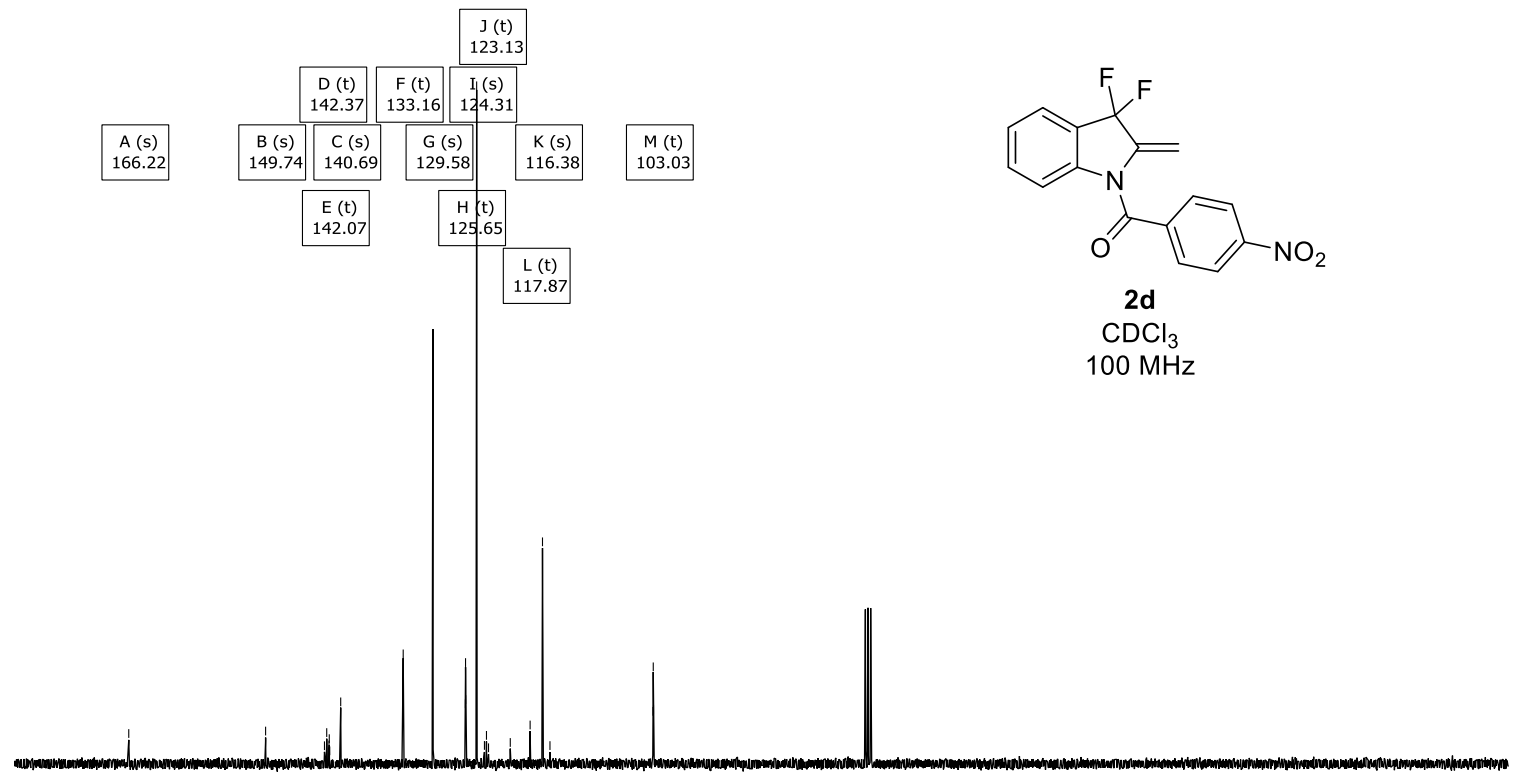




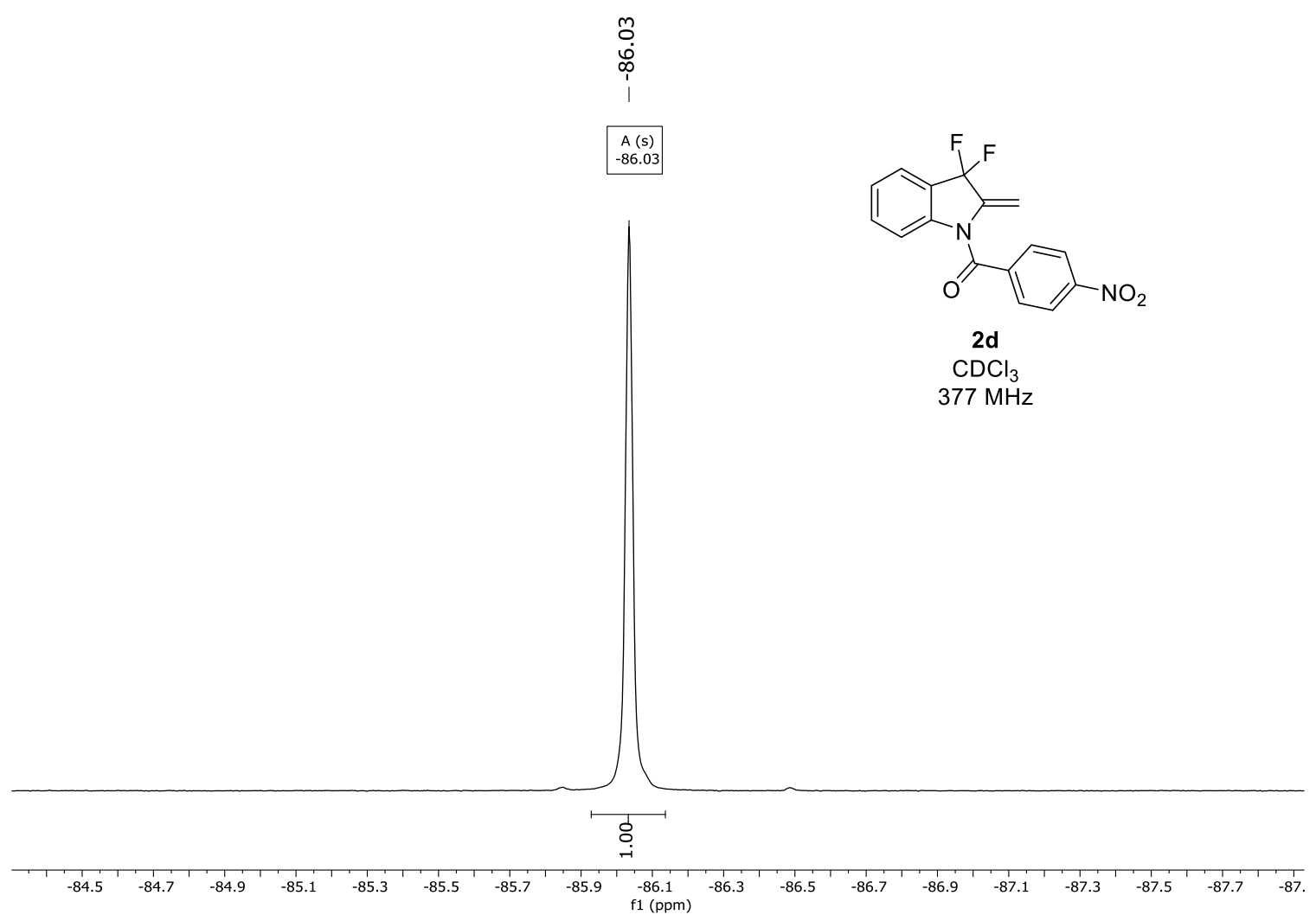




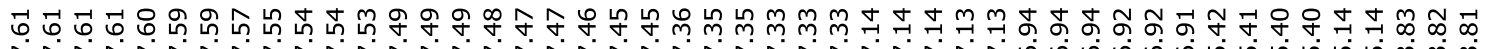

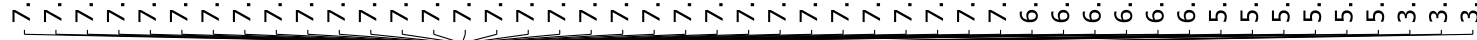

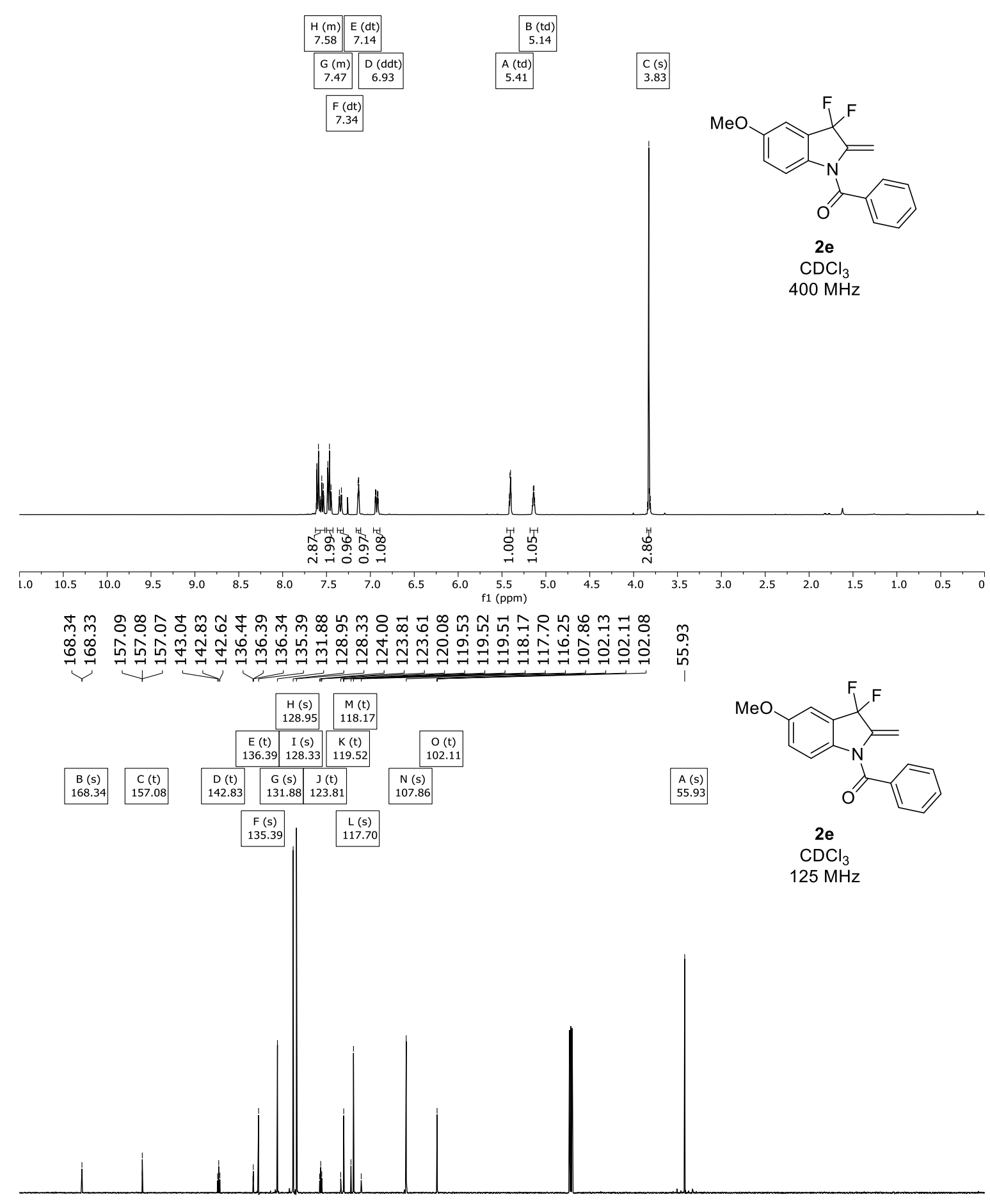

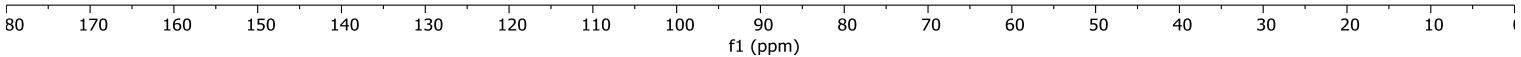




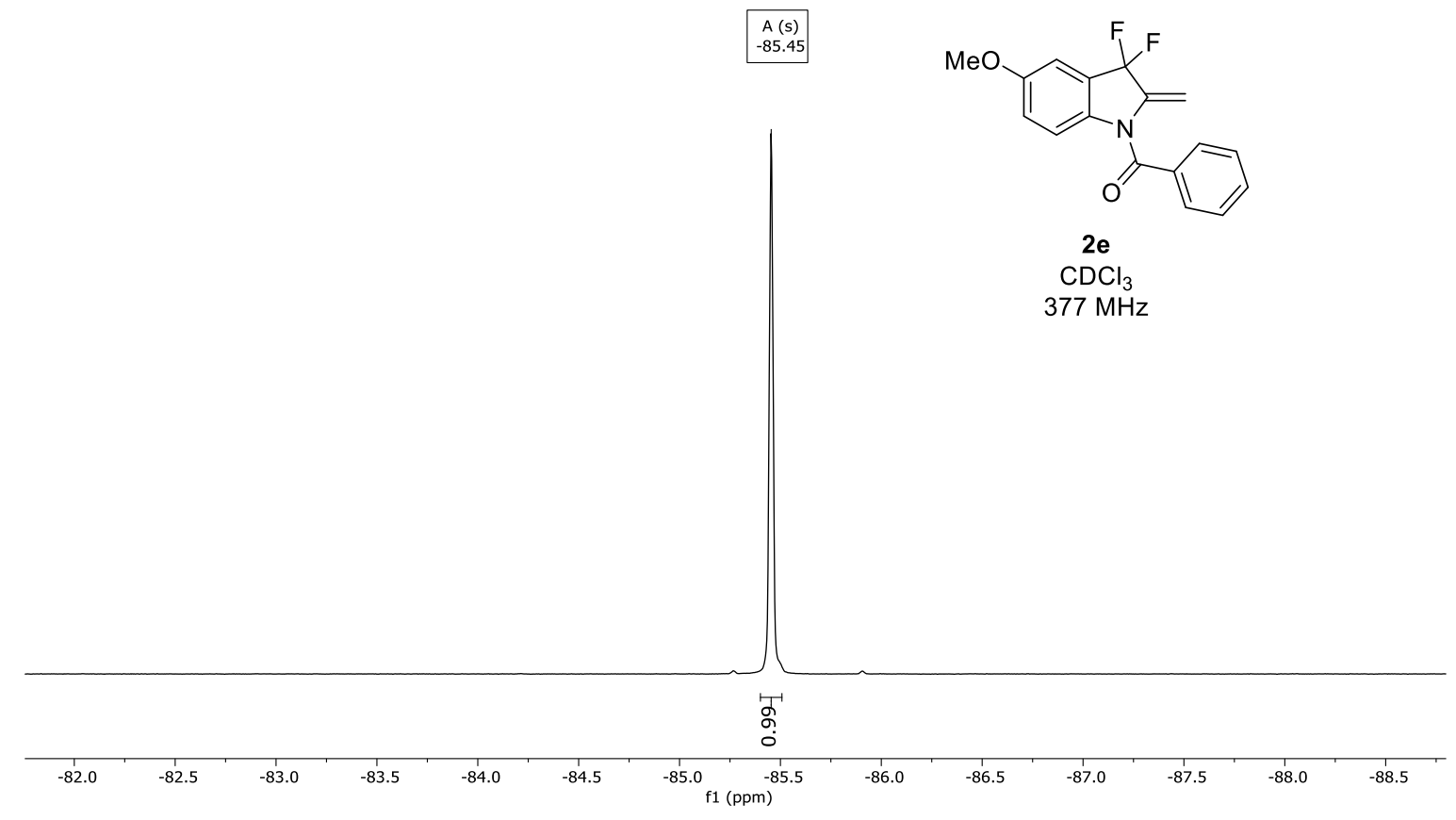




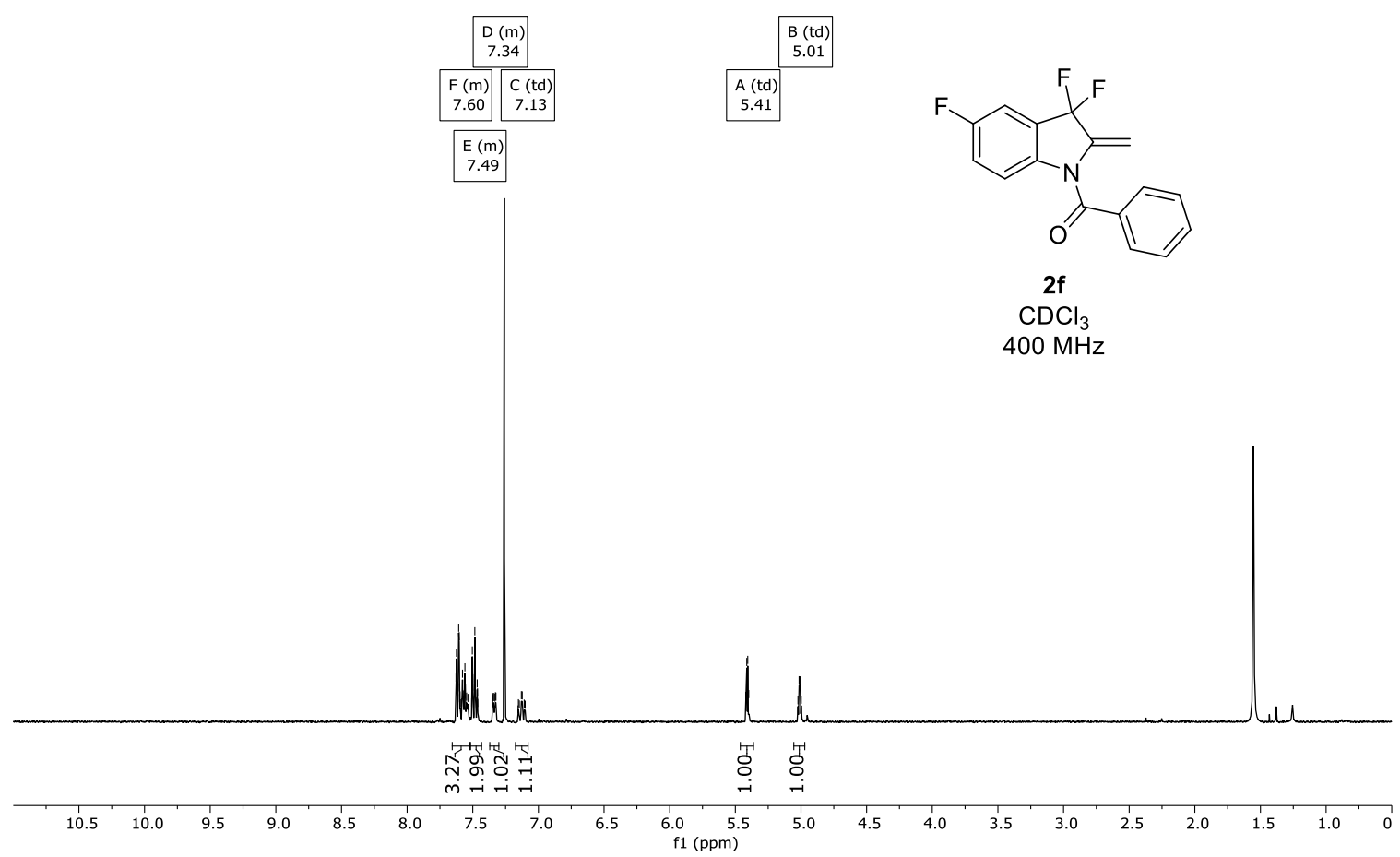

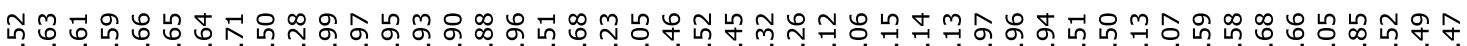

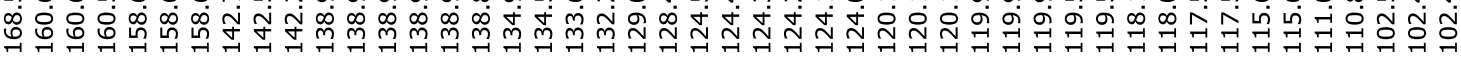

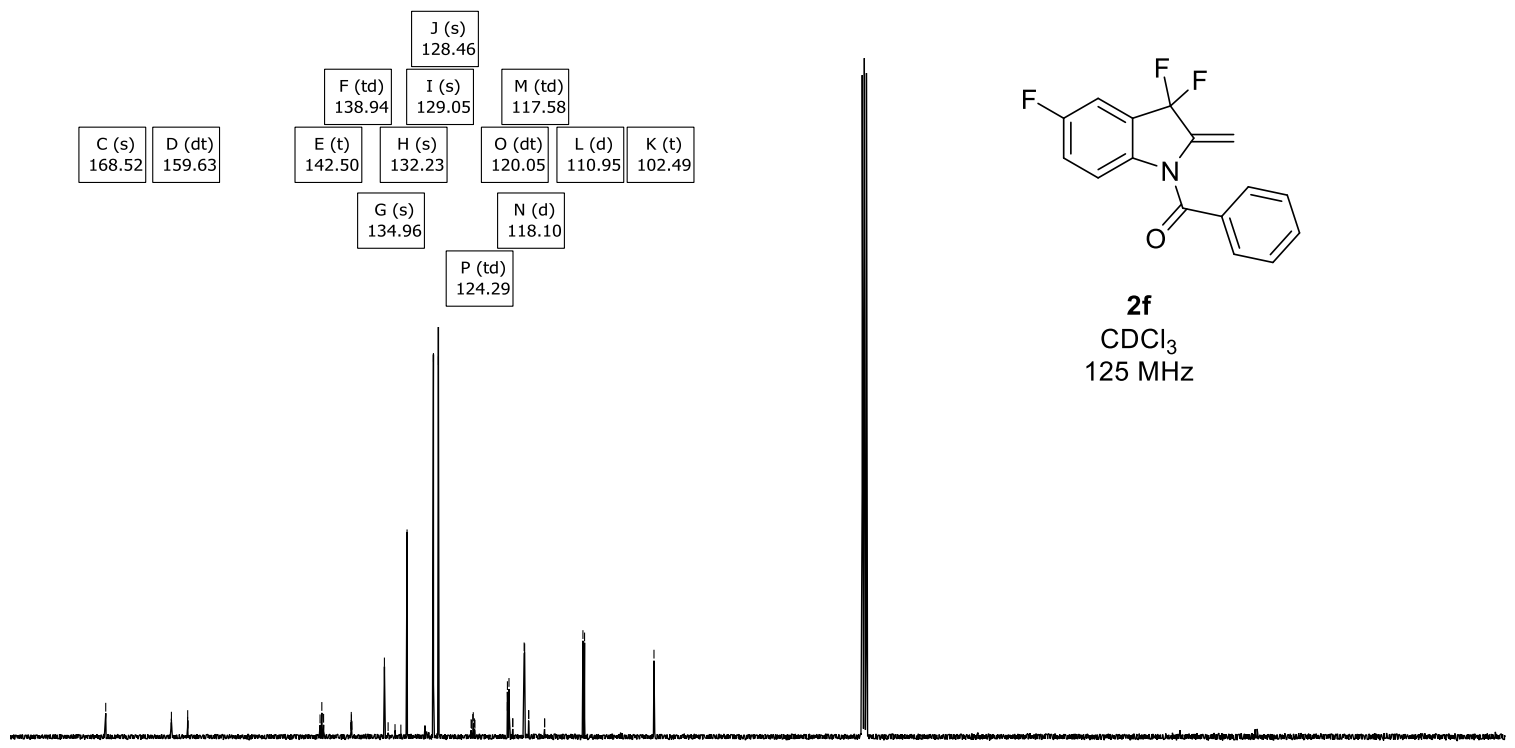

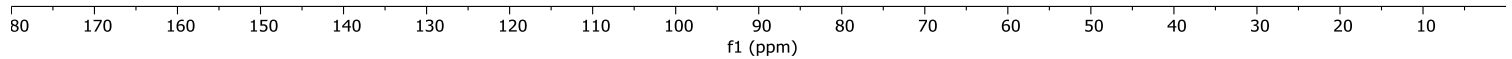




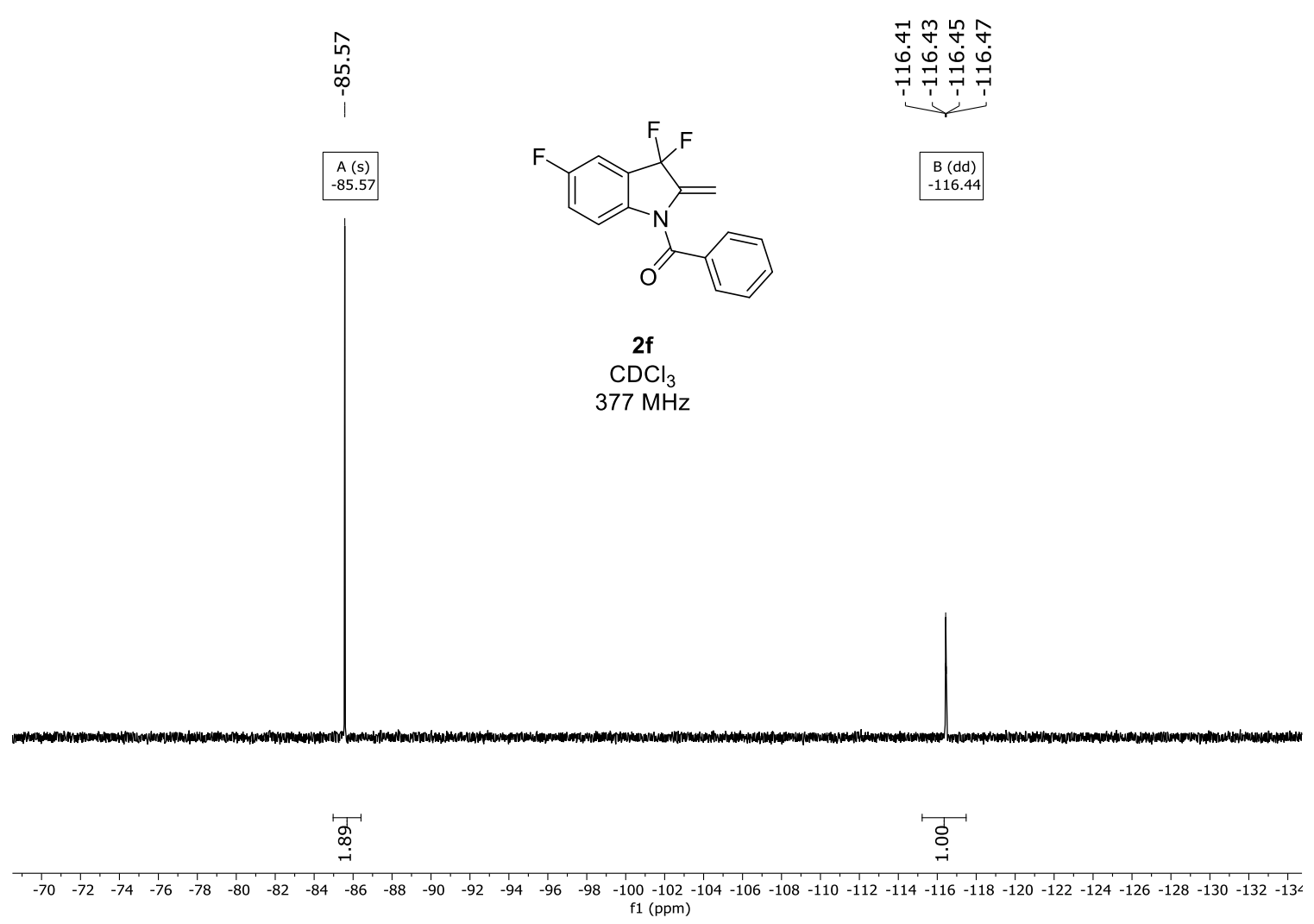




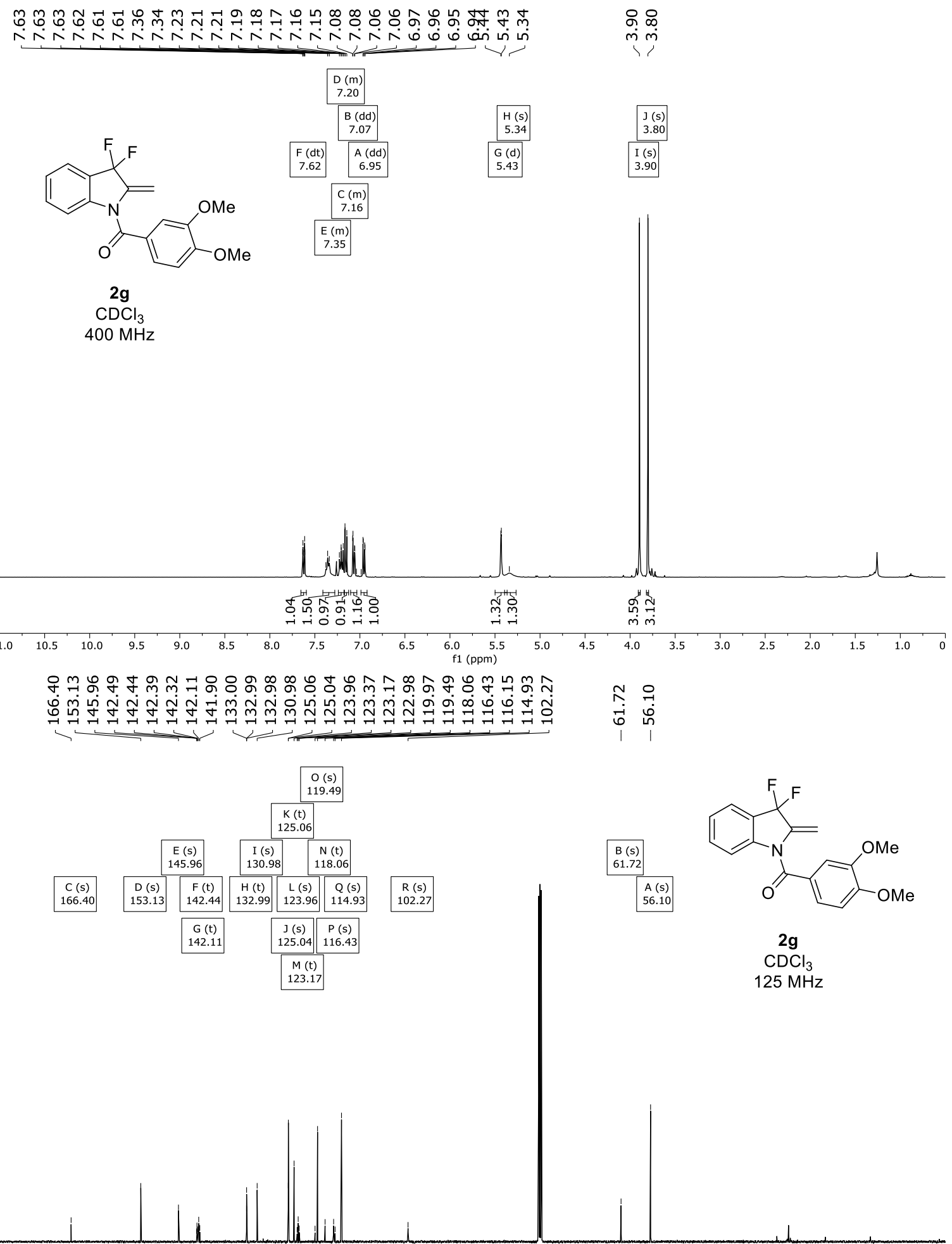

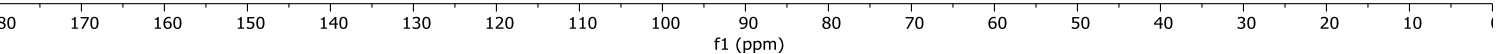




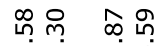

م)

111

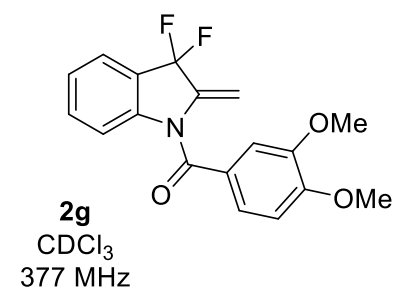

A (d) B (d)

$-82.94 \quad-86.23$

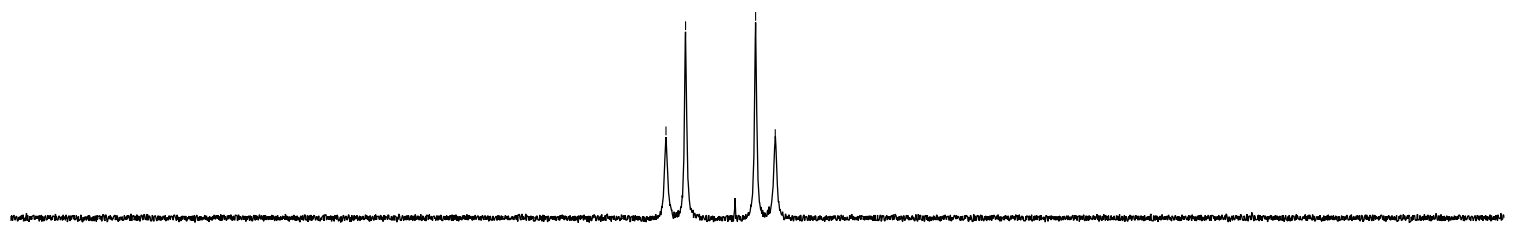

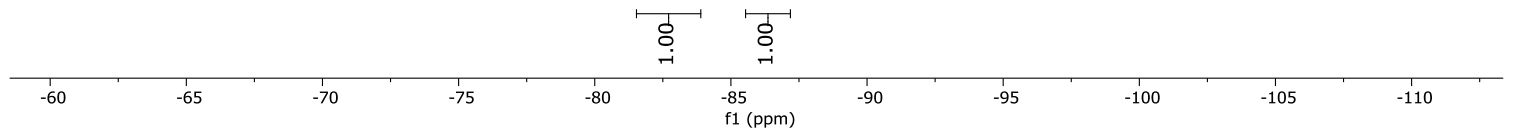




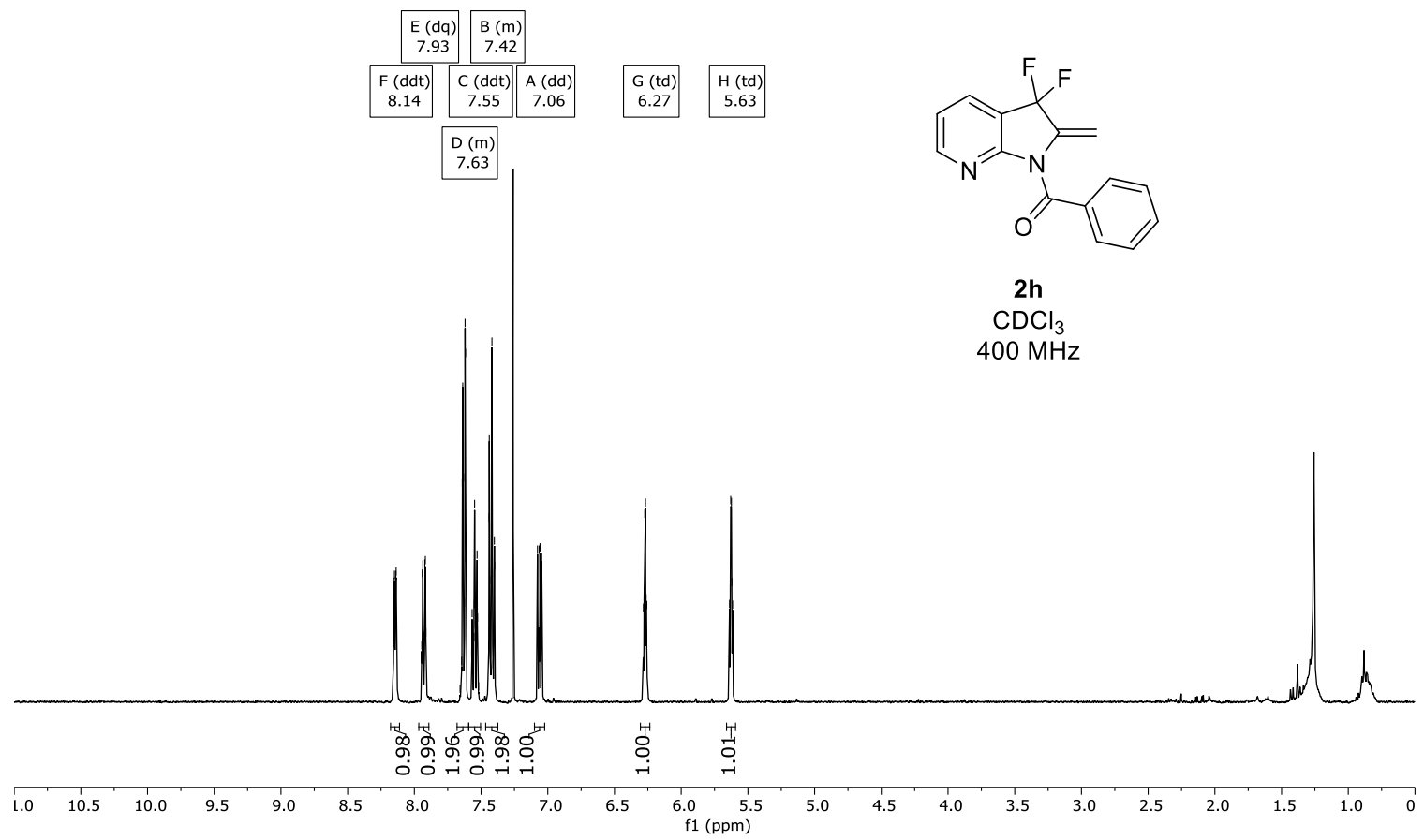

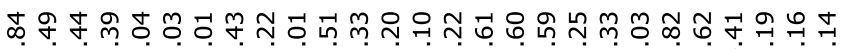

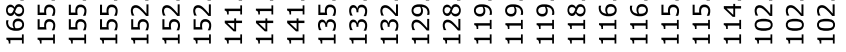

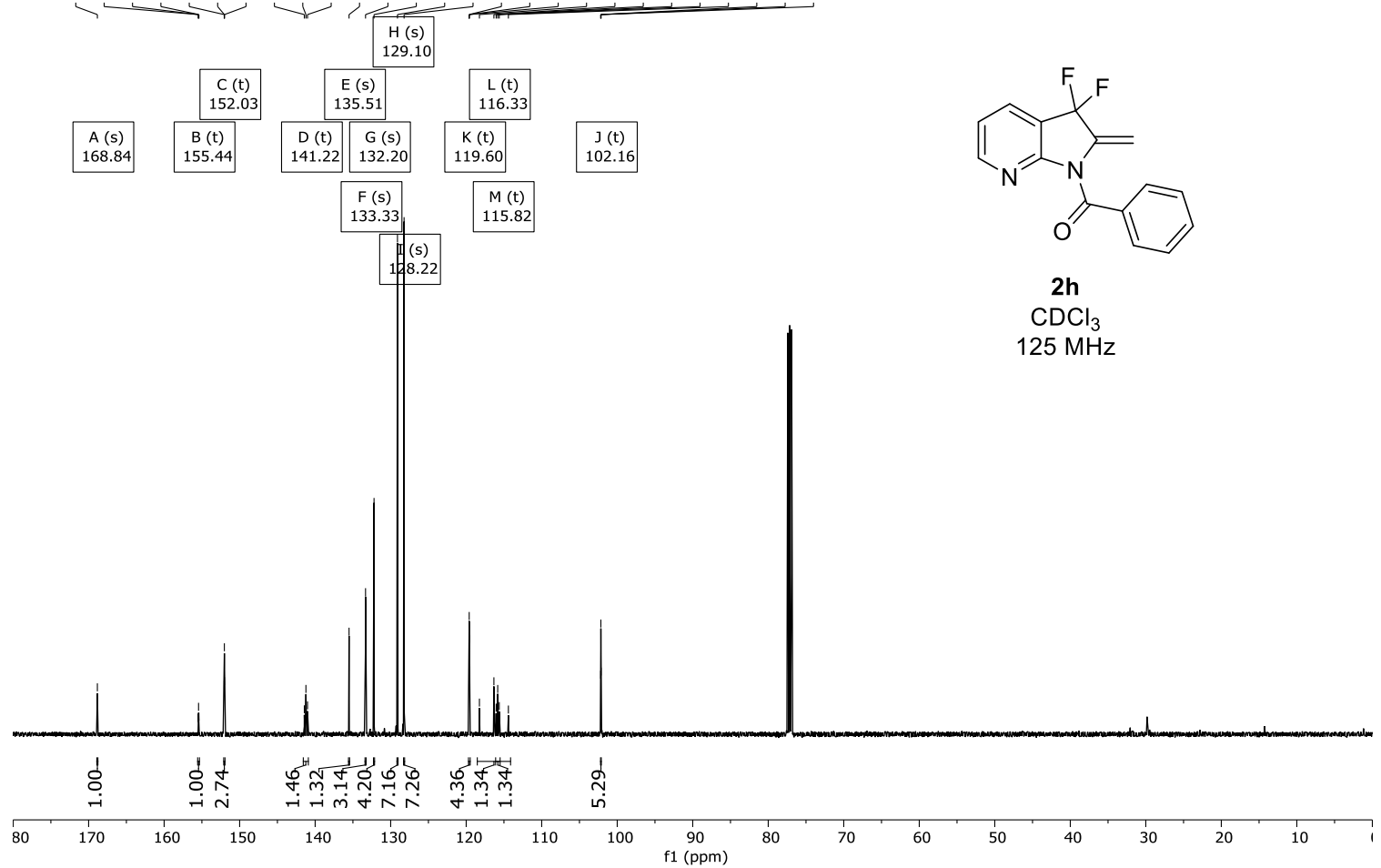




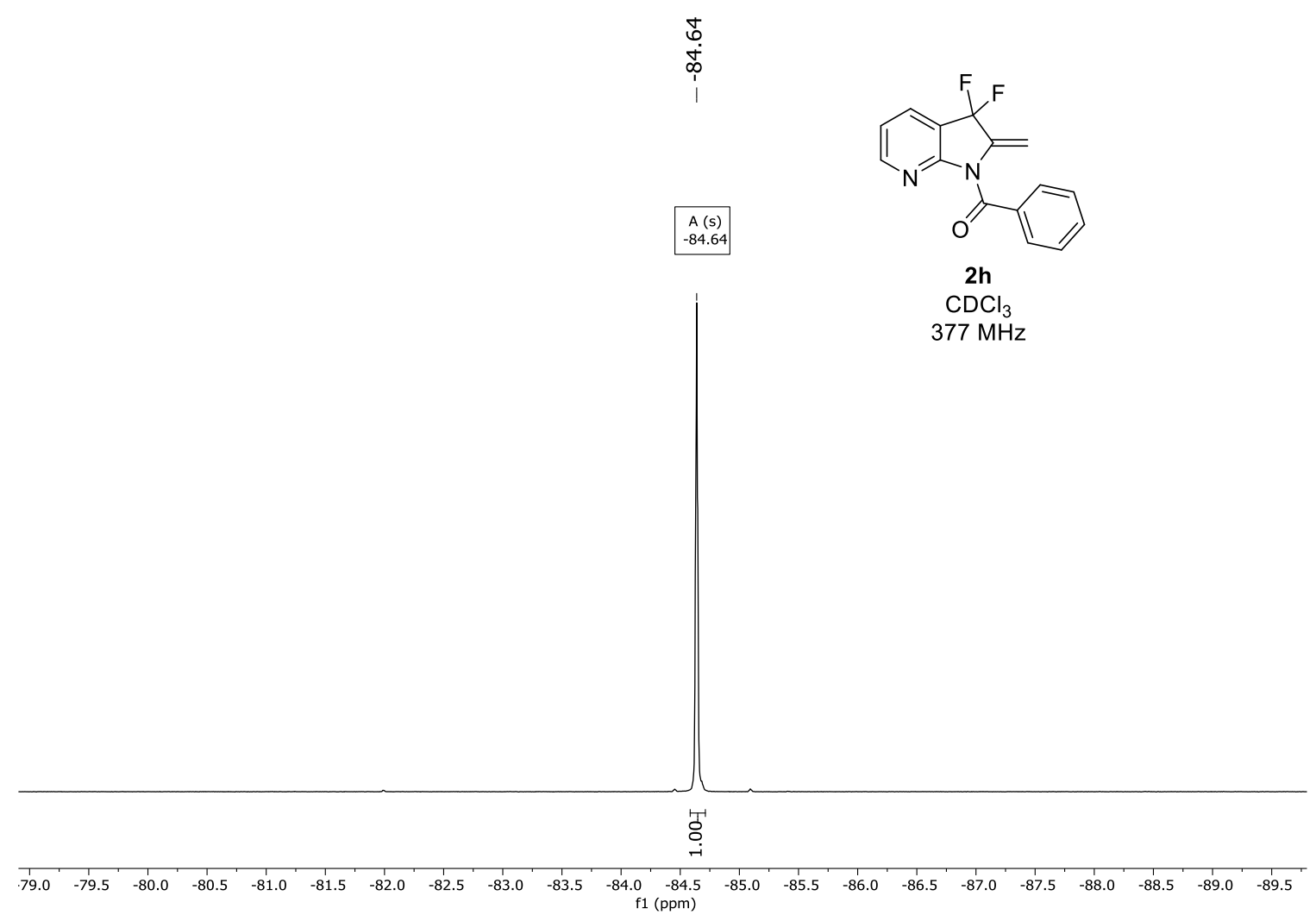


<smiles>O=C(c1ccccc1)c1c(F)c2ccccc2n1CN1CCOCC1</smiles>

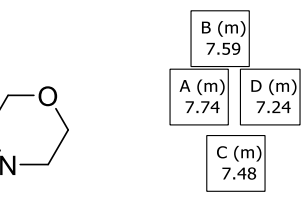

$\mathrm{Bz}$
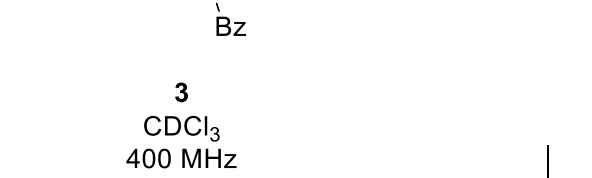

$400 \mathrm{MHz}$

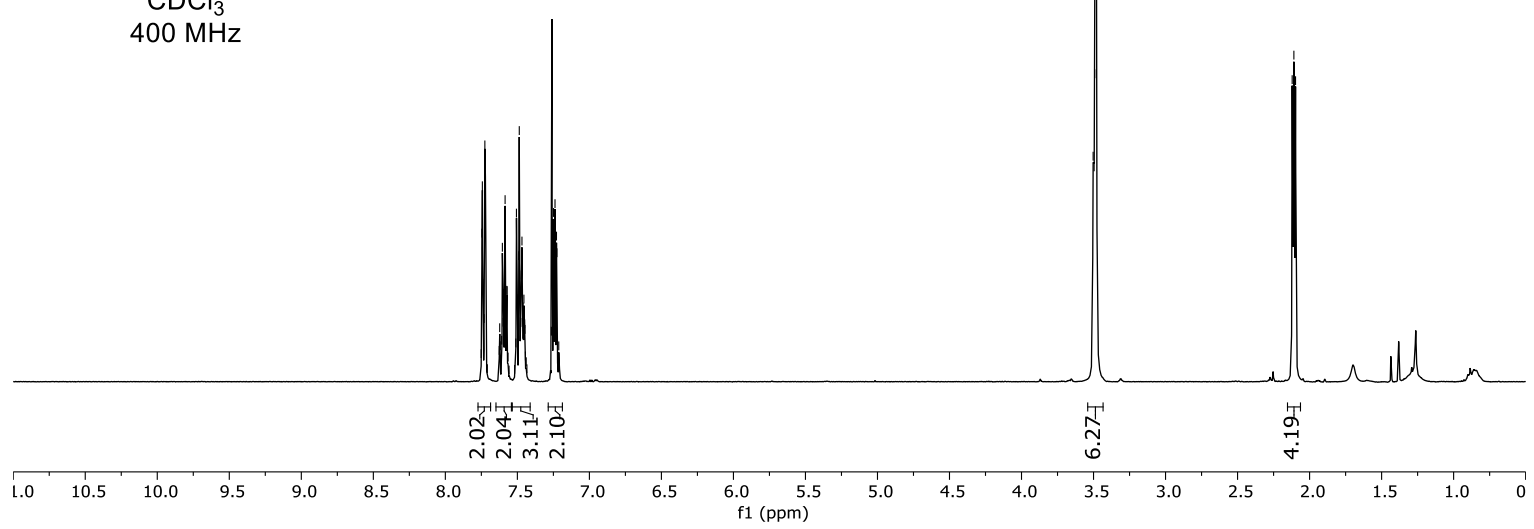

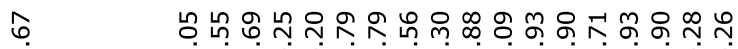

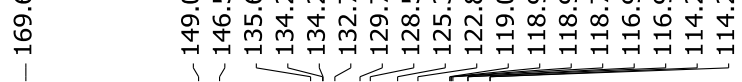

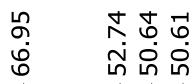

1 $>$
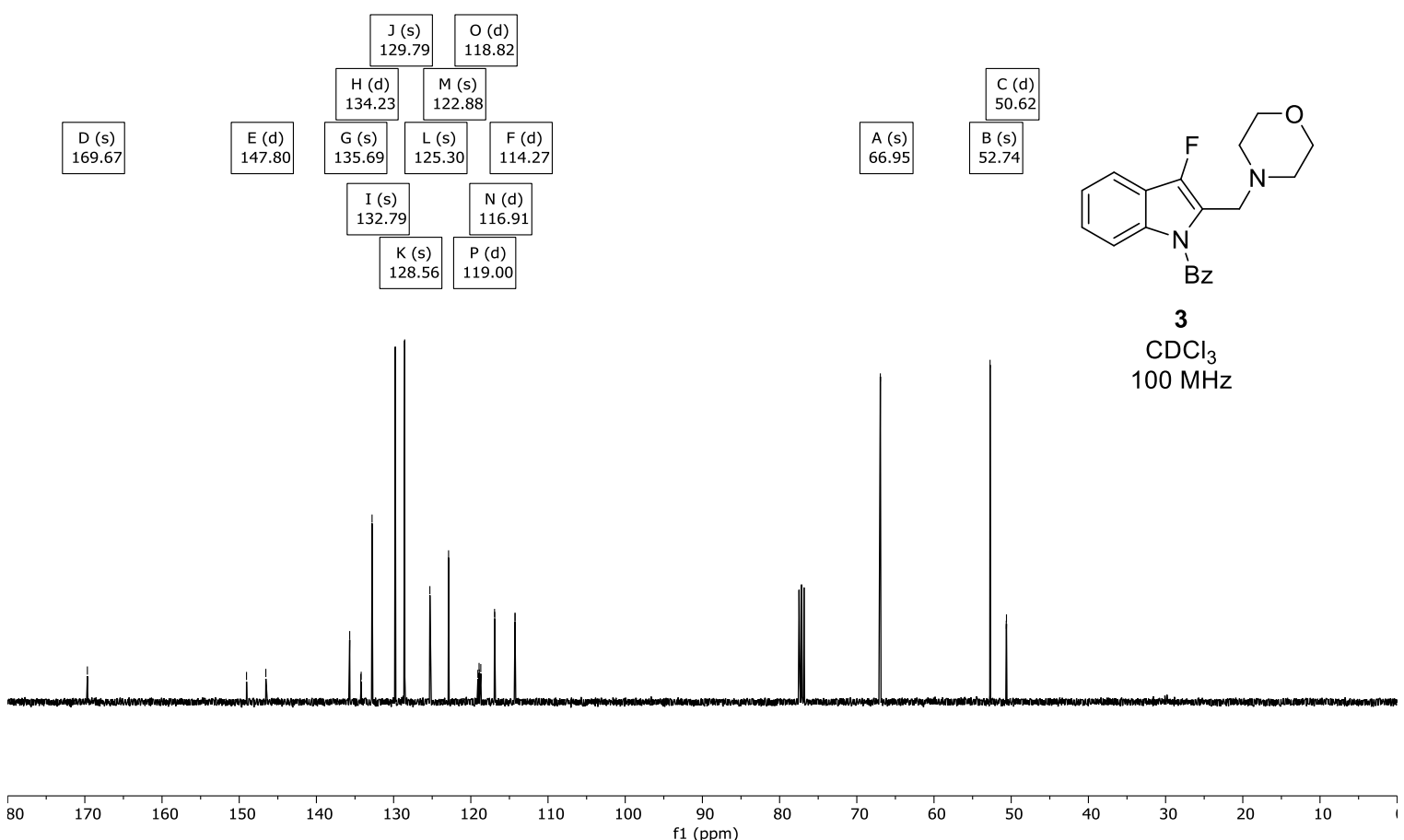

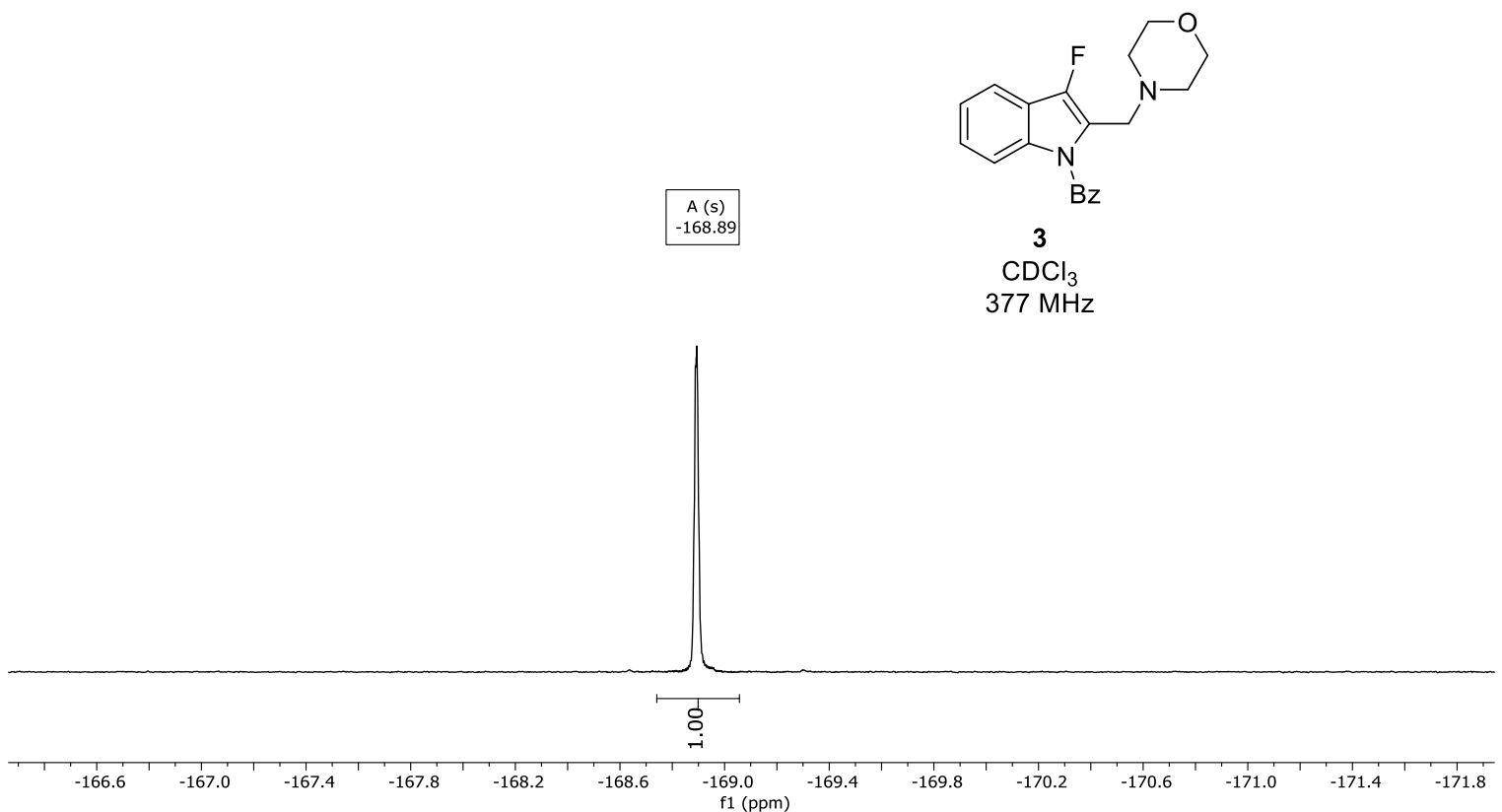


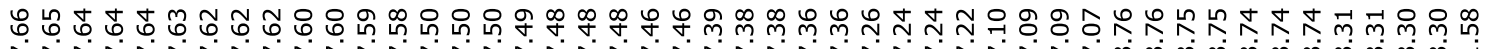

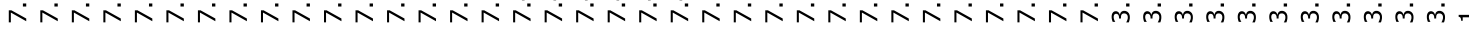
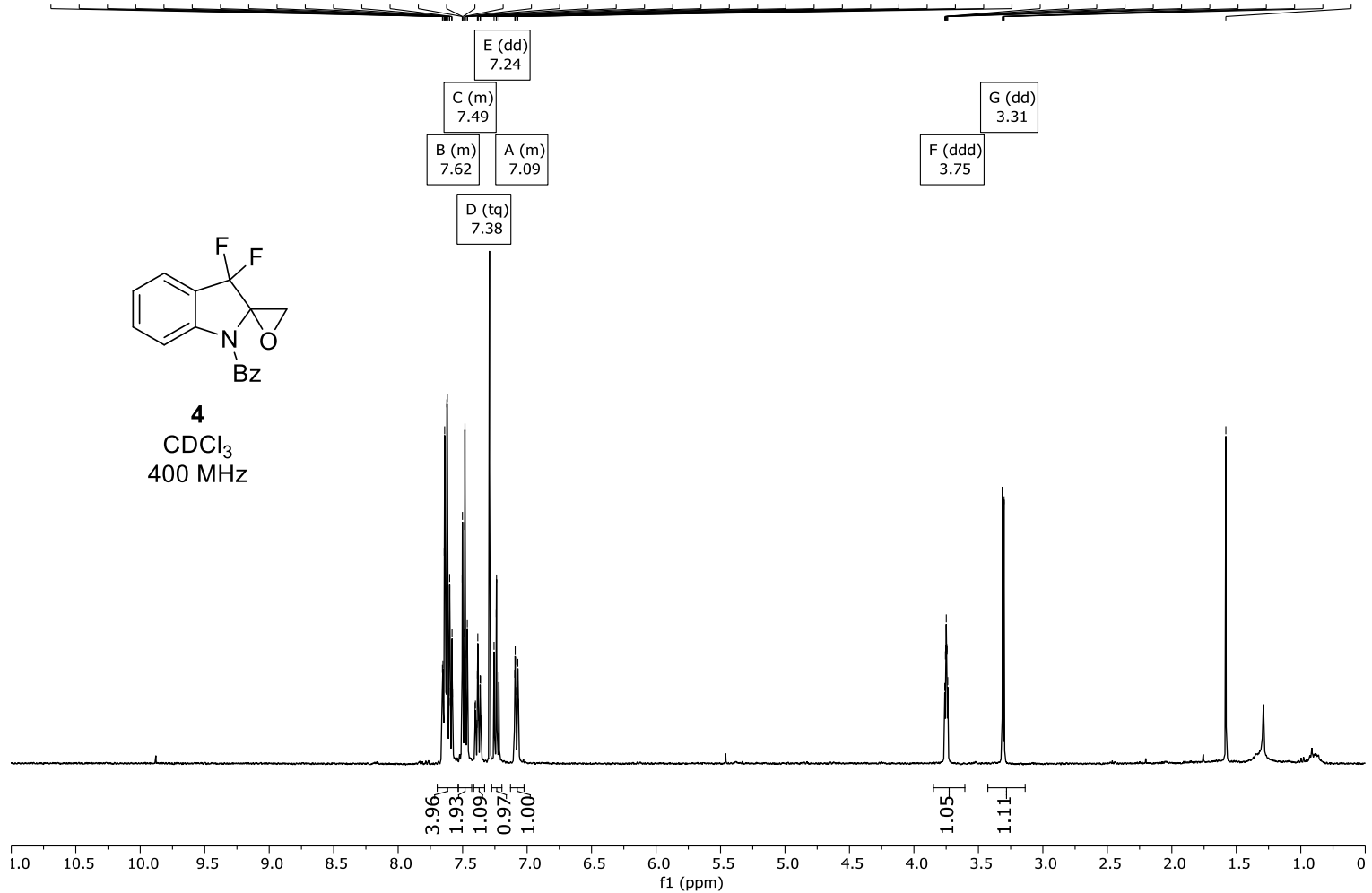

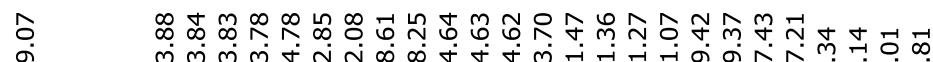

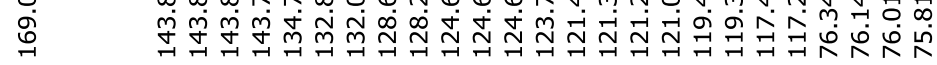

จุํา

$\underbrace{\infty}_{\substack{\infty \\ \infty}}$

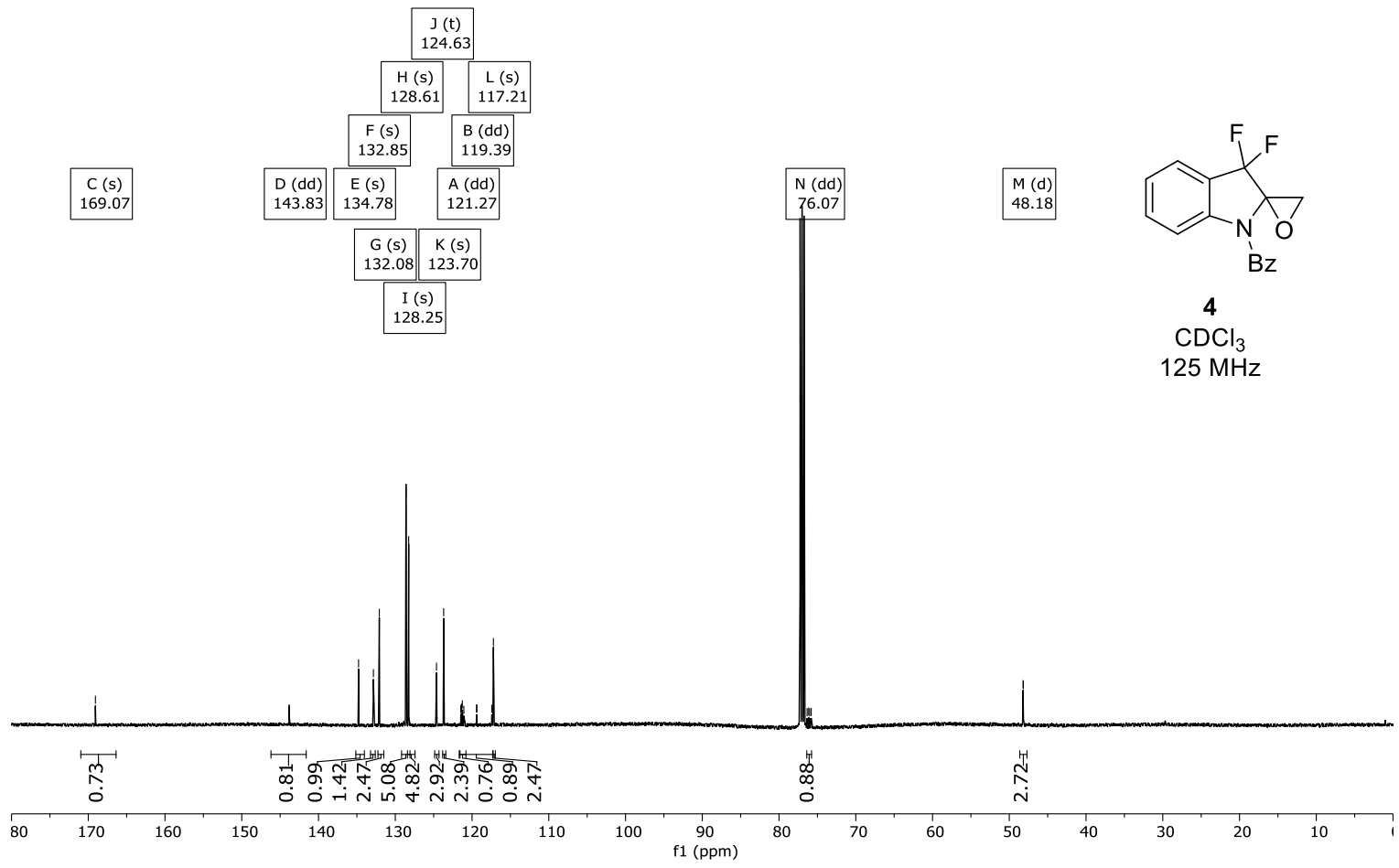



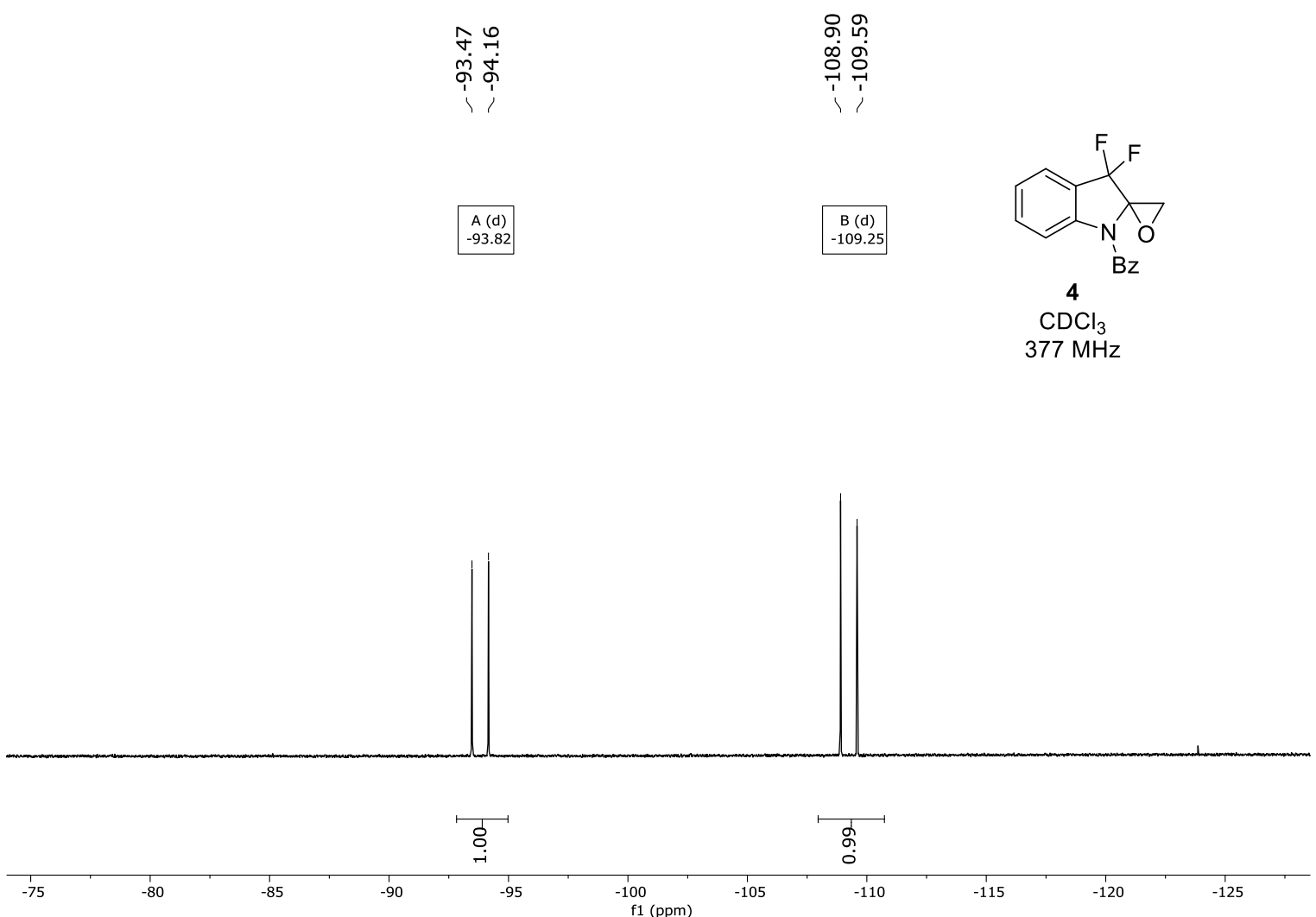


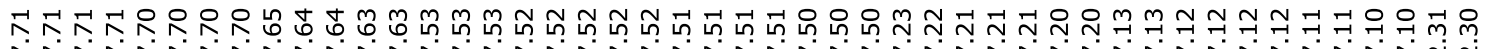

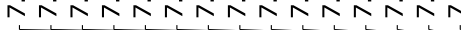

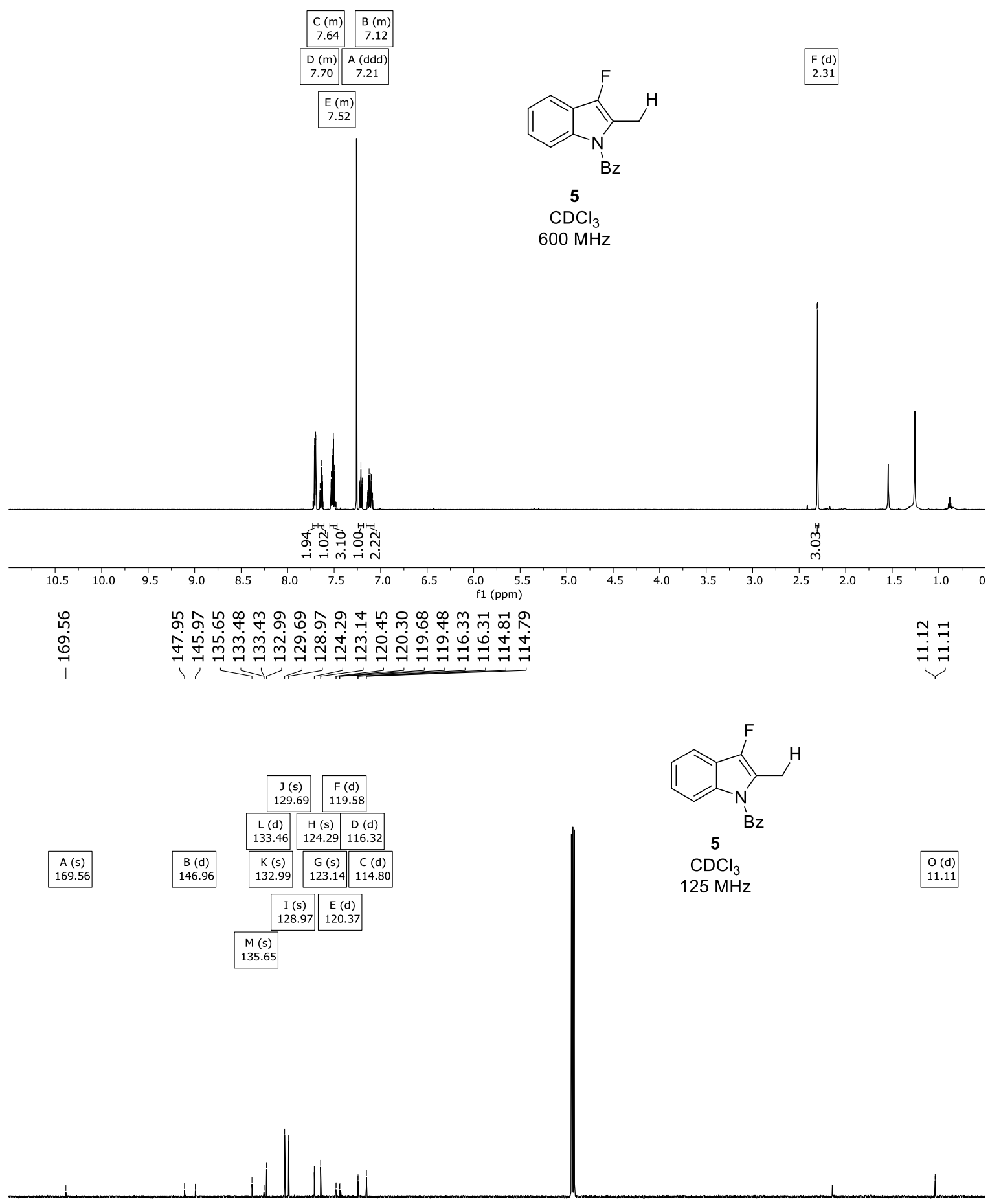

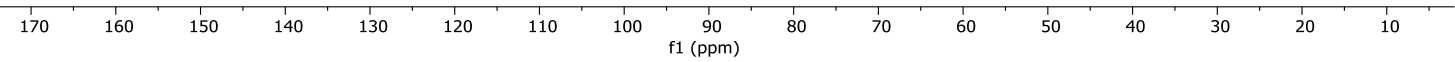




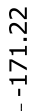

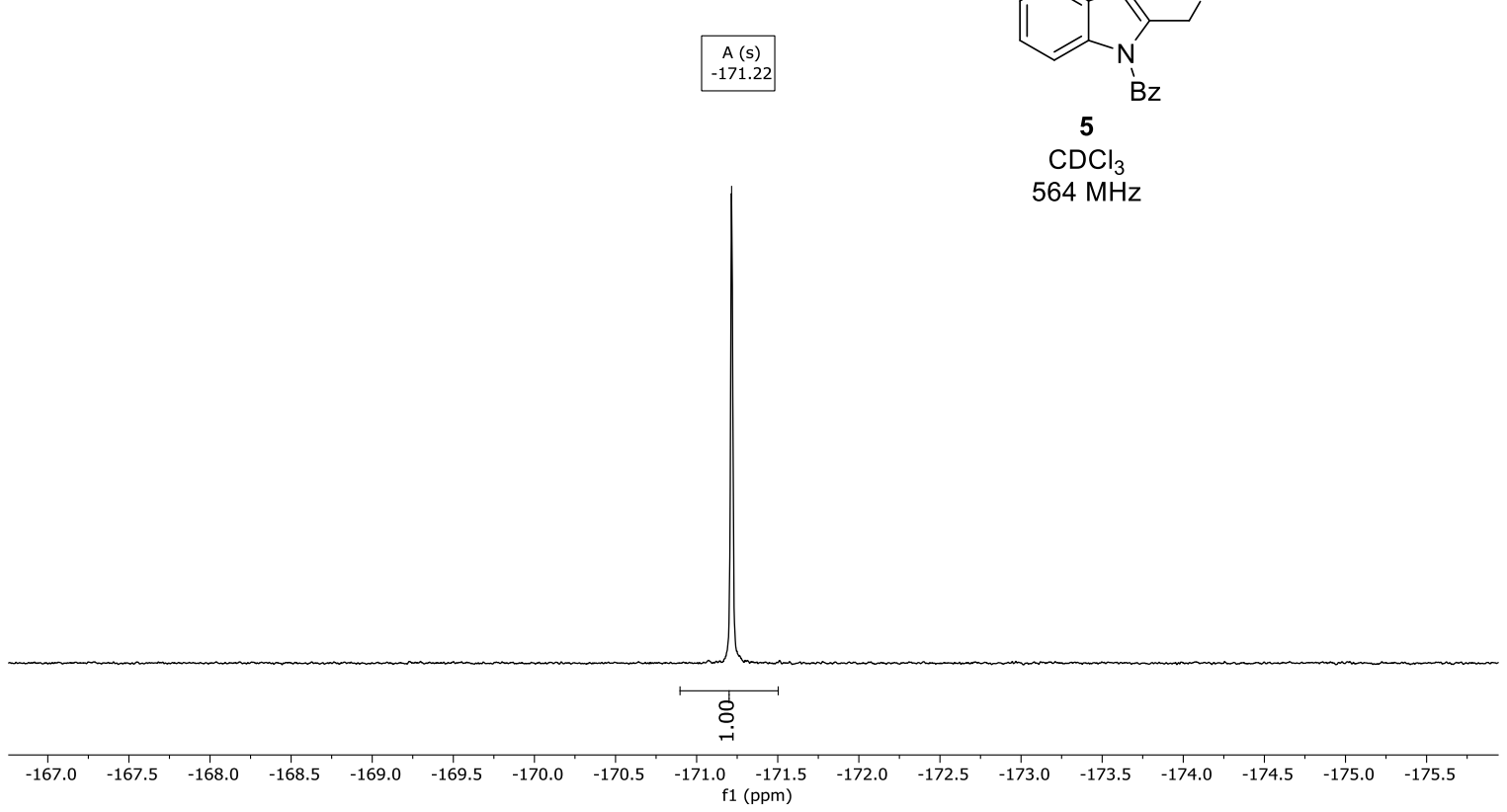




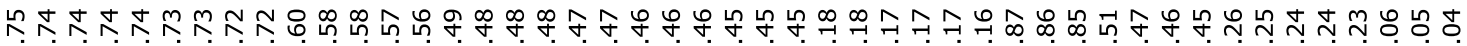

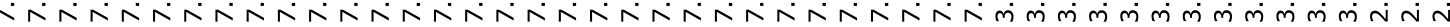

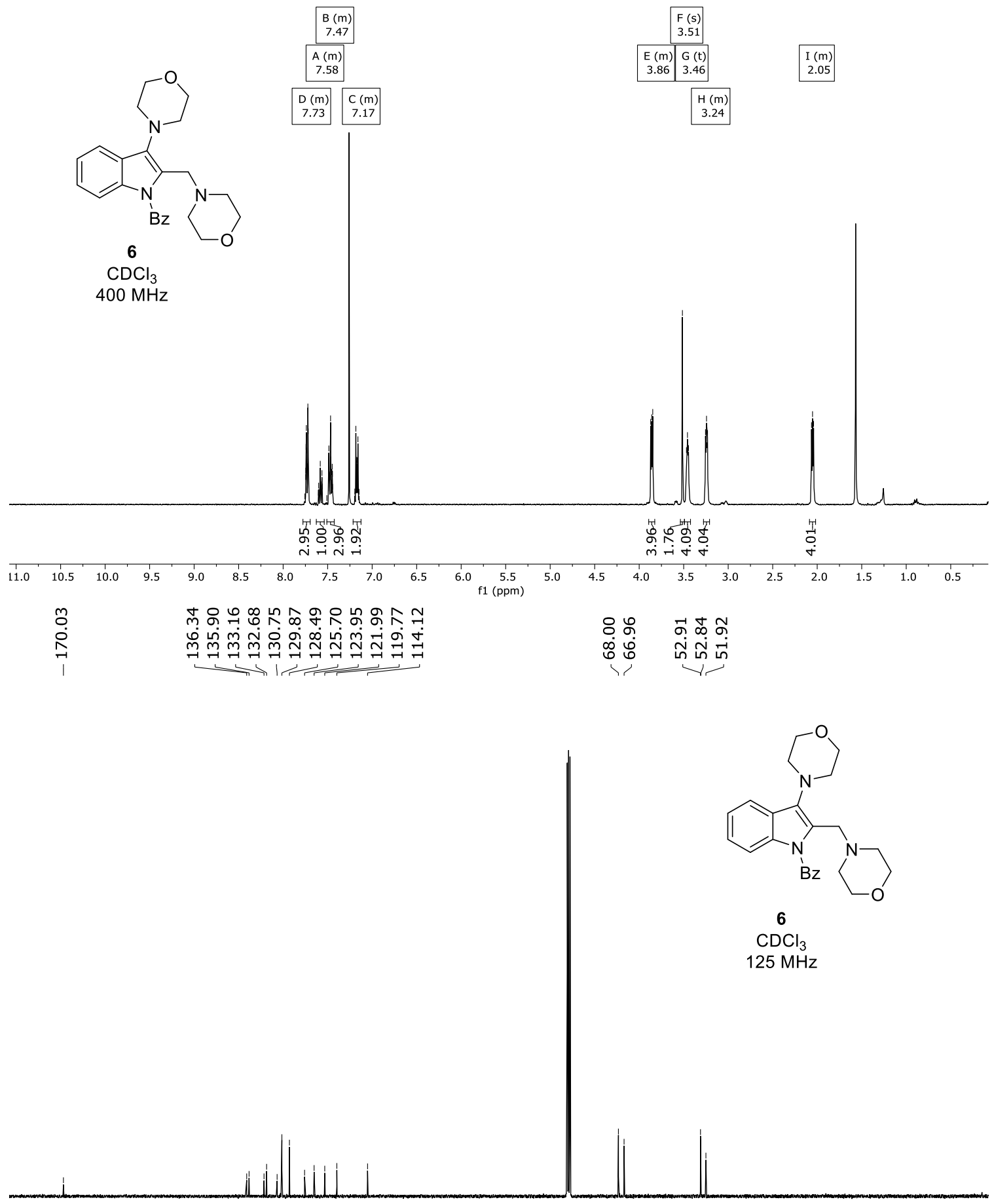

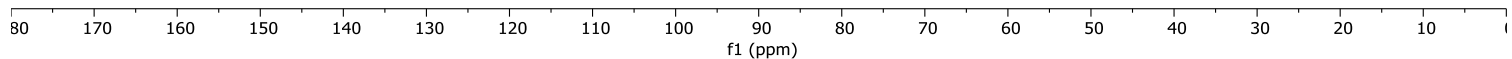



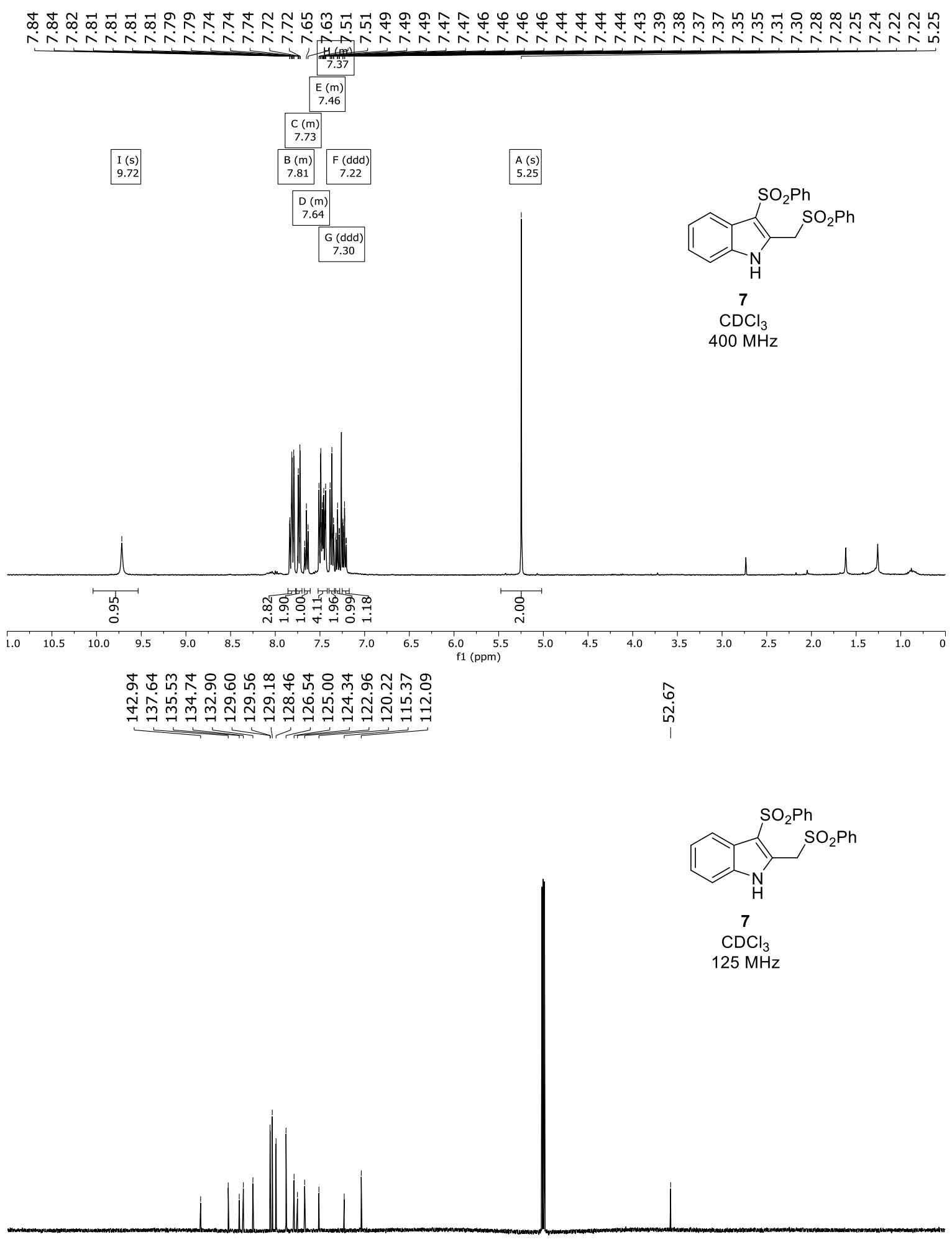

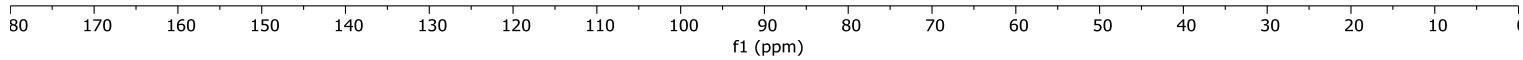




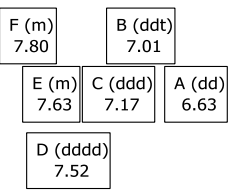

$$
\begin{array}{|c|}
\hline \begin{array}{c}
H(d d) \\
3.74
\end{array} \\
\hline G \text { (dqd) } \\
4.01 \\
\hline
\end{array}
$$<smiles>O=COCc1c(F)c2ccccc2n1Cc1ccccc1</smiles>

8 $\mathrm{CDCl}_{3}$ $400 \mathrm{MHz}$

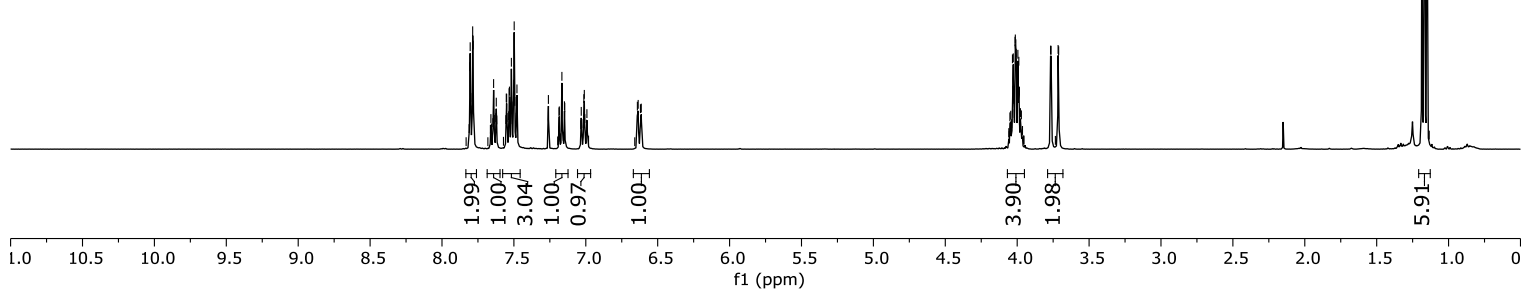

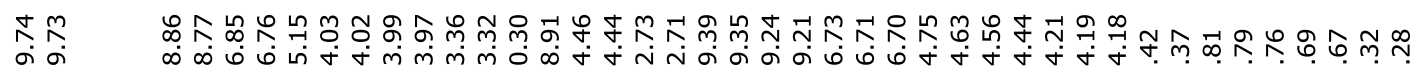

oi o

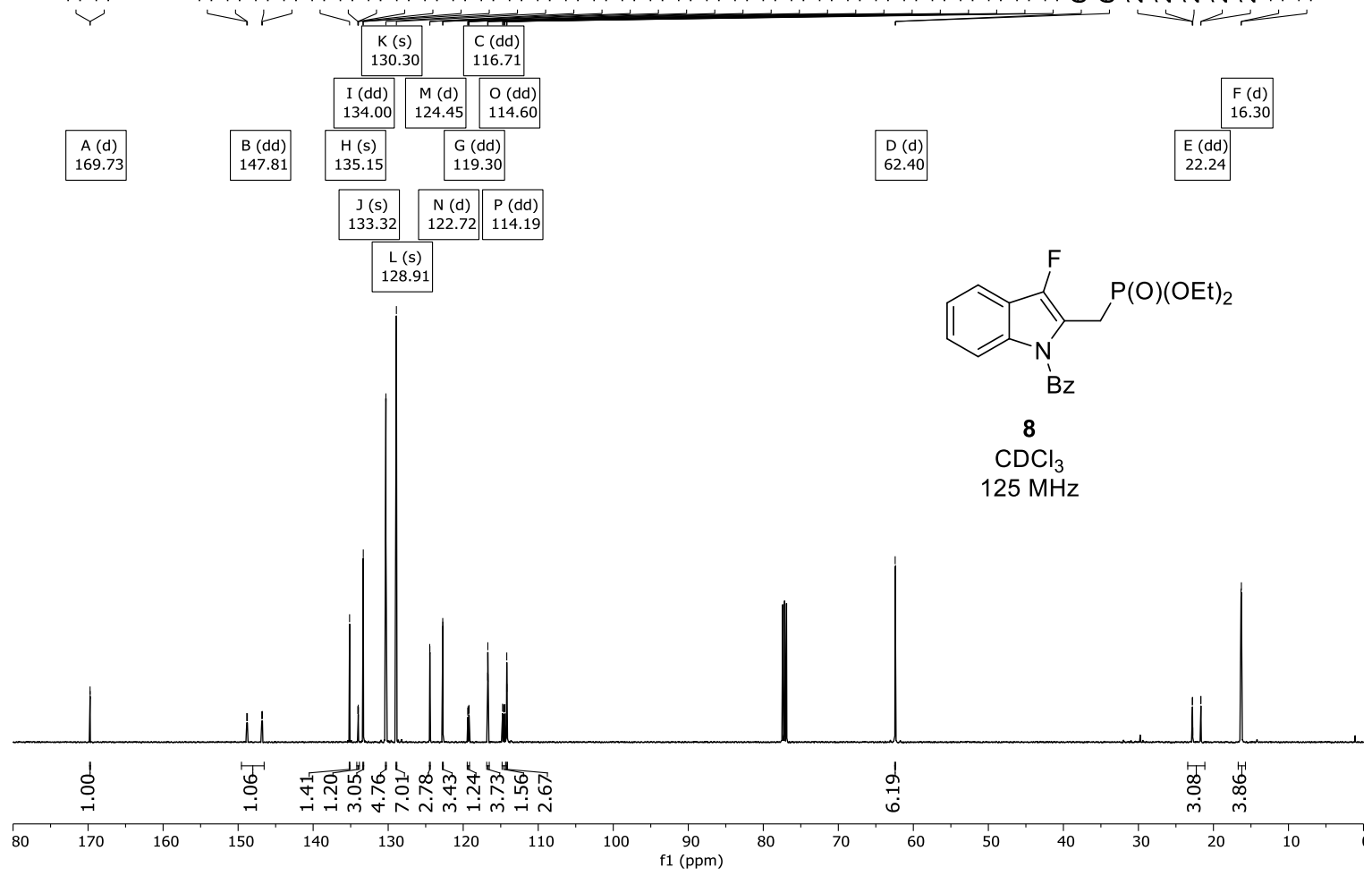


ํㅜㅇ

我

')

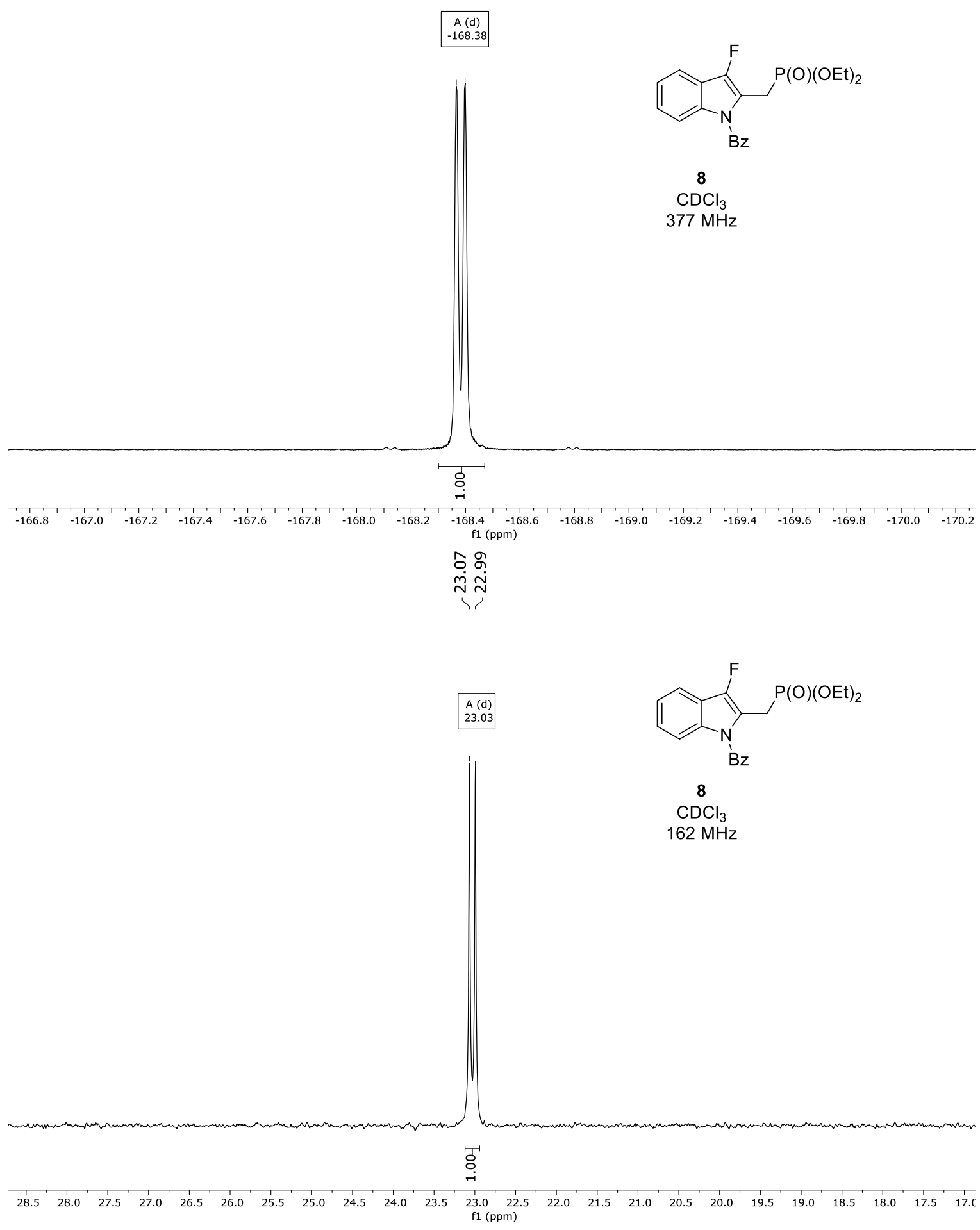




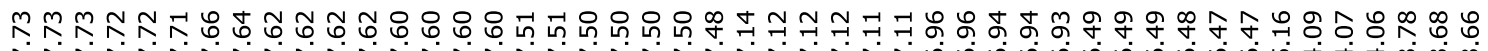

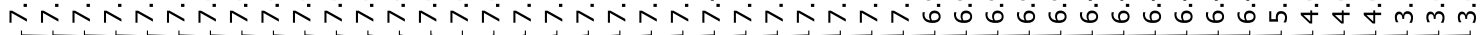
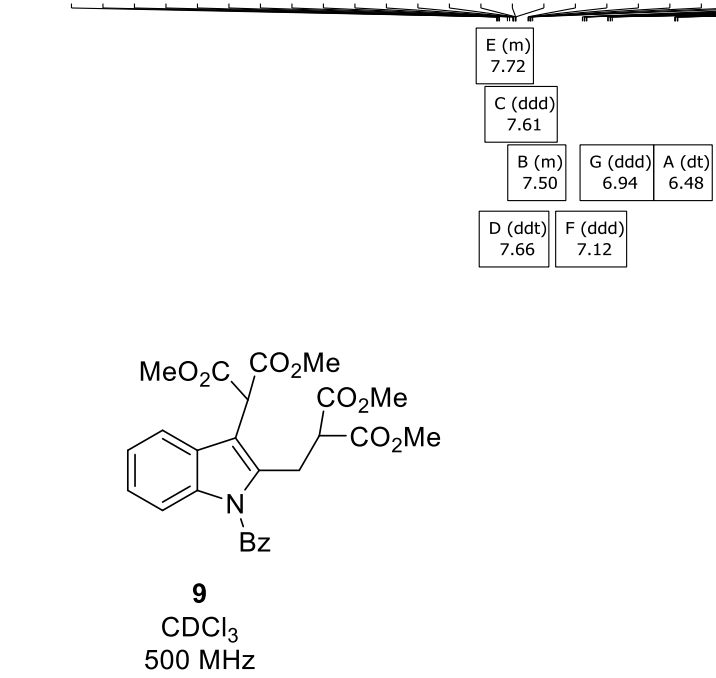

9

$\mathrm{CDCl}_{3}$

$500 \mathrm{MHz}$

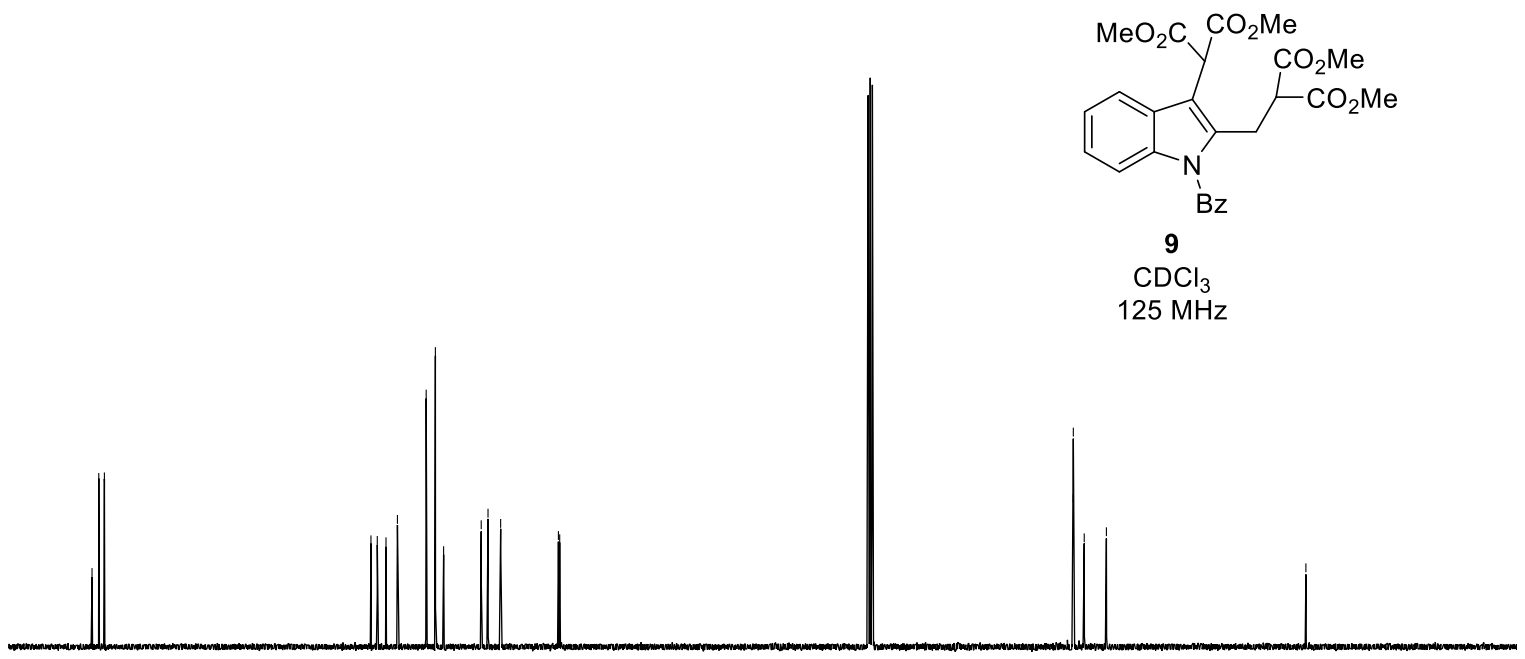

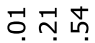

웡

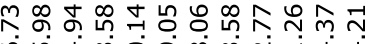

ᄀi.

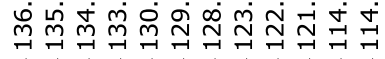

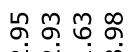

กู่ กิ่ ที่

$\stackrel{\infty}{\rightarrow-1}$

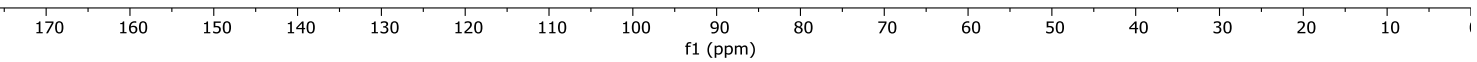




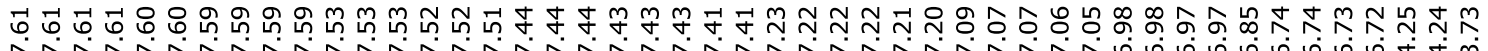

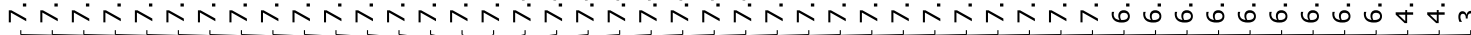

$$
\begin{aligned}
& \begin{array}{|c|c|}
\hline H(m) & D \text { (ddd) } \\
7.60 & 7.07 \\
\hline
\end{array} \\
& \text { E (ddd) } B(m)
\end{aligned}
$$

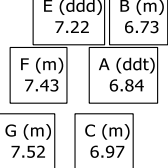

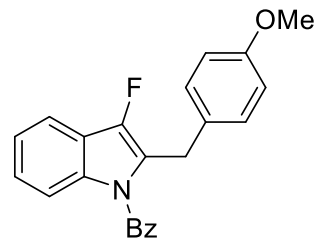

12

$\mathrm{CDCl}_{3}$

$500 \mathrm{MHz}$

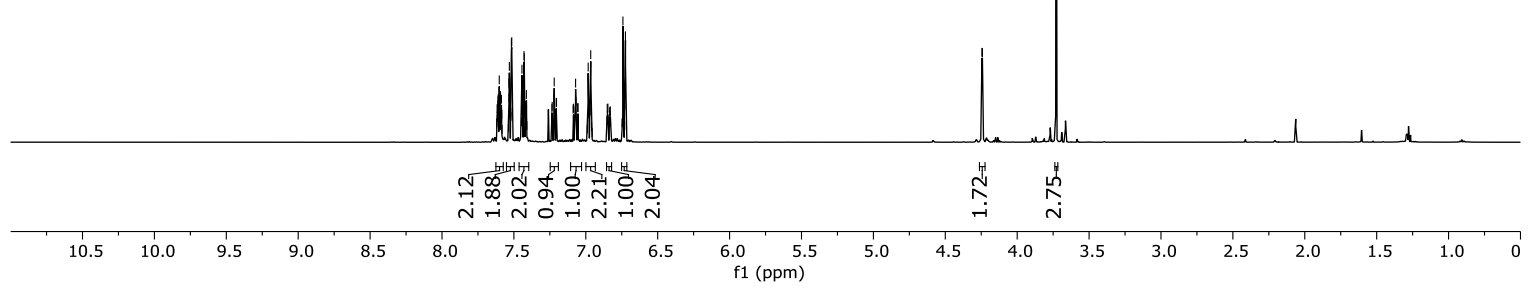

₹. $\quad \hat{\tau} \quad$ m

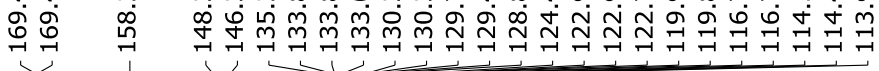

।

\begin{tabular}{|c|c|}
\hline$N(s)$ & $I(d)$ \\
129.43 & 119.87 \\
\hline
\end{tabular}

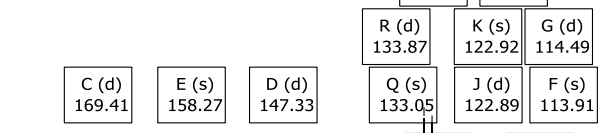
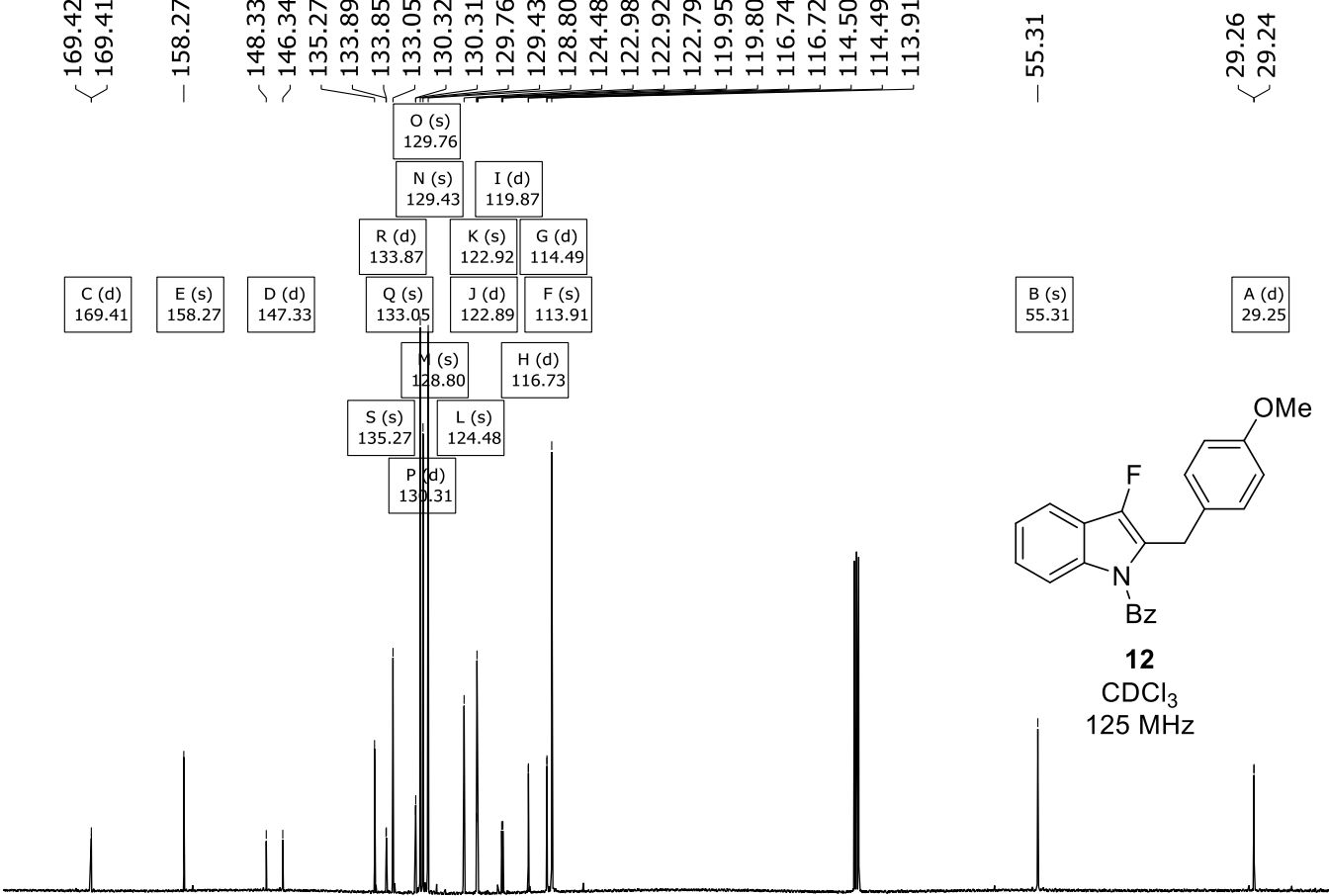

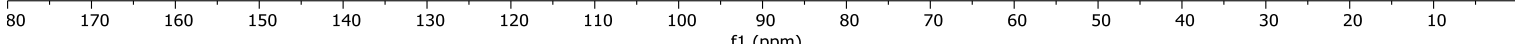




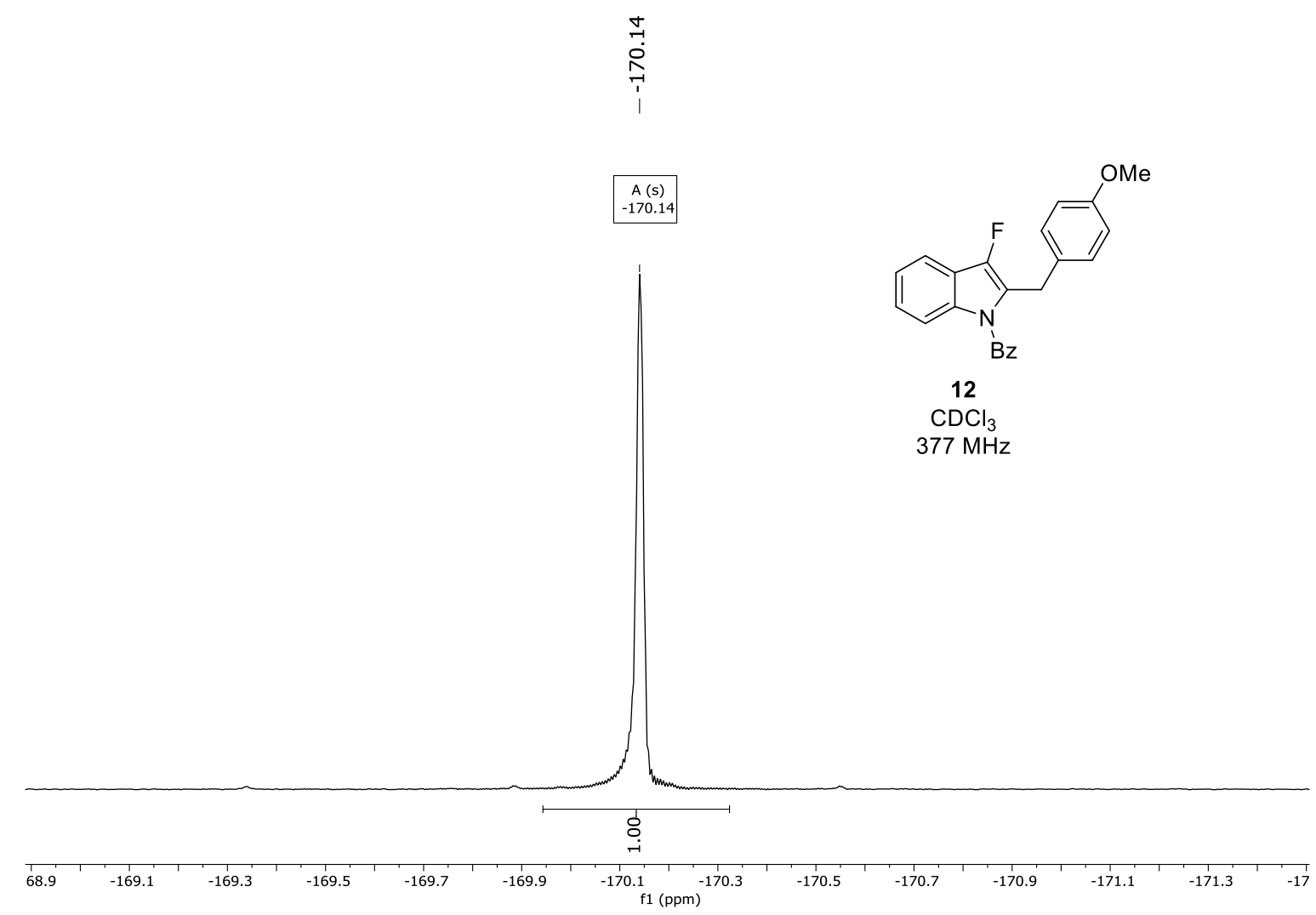



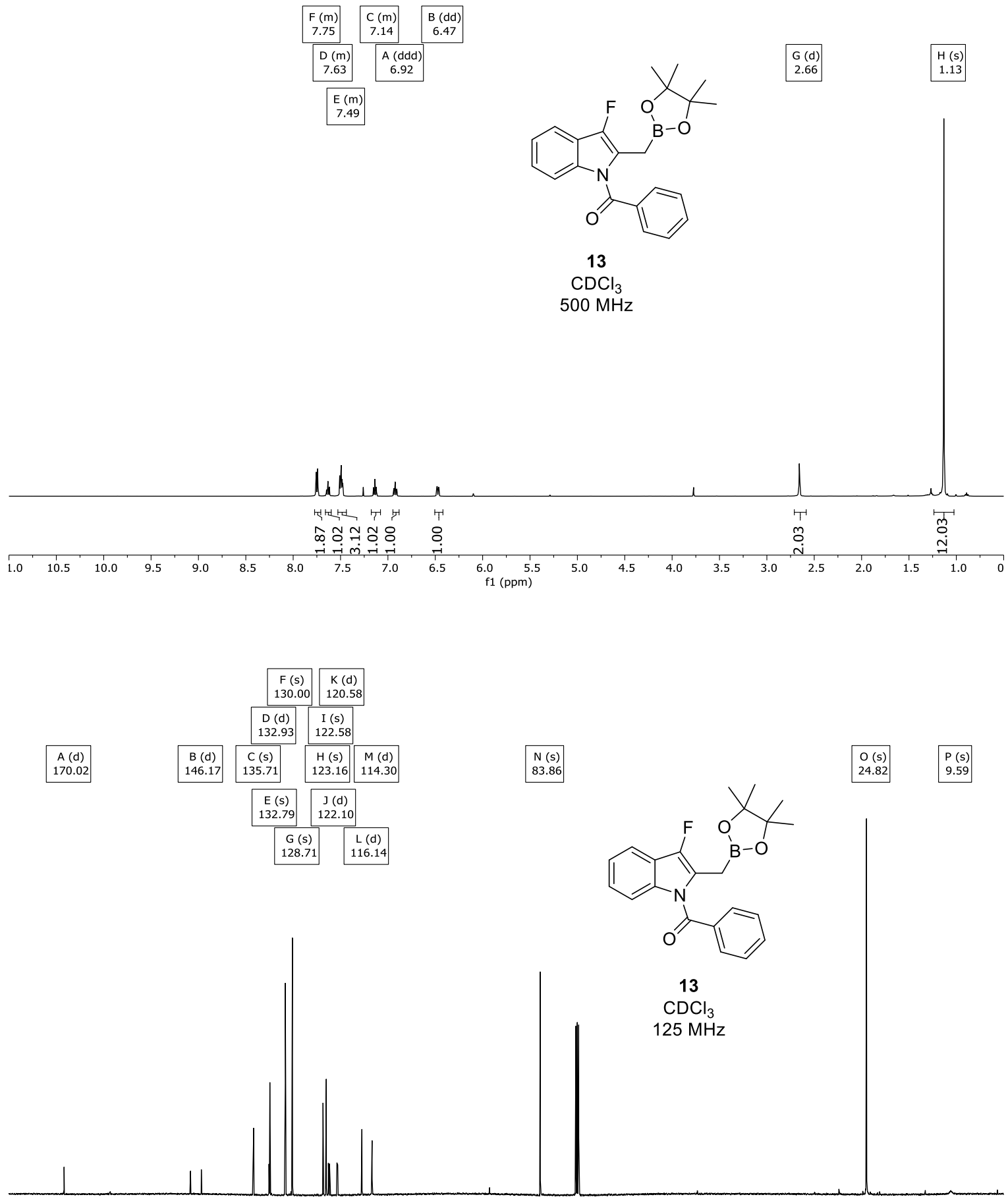

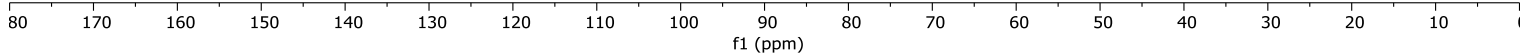




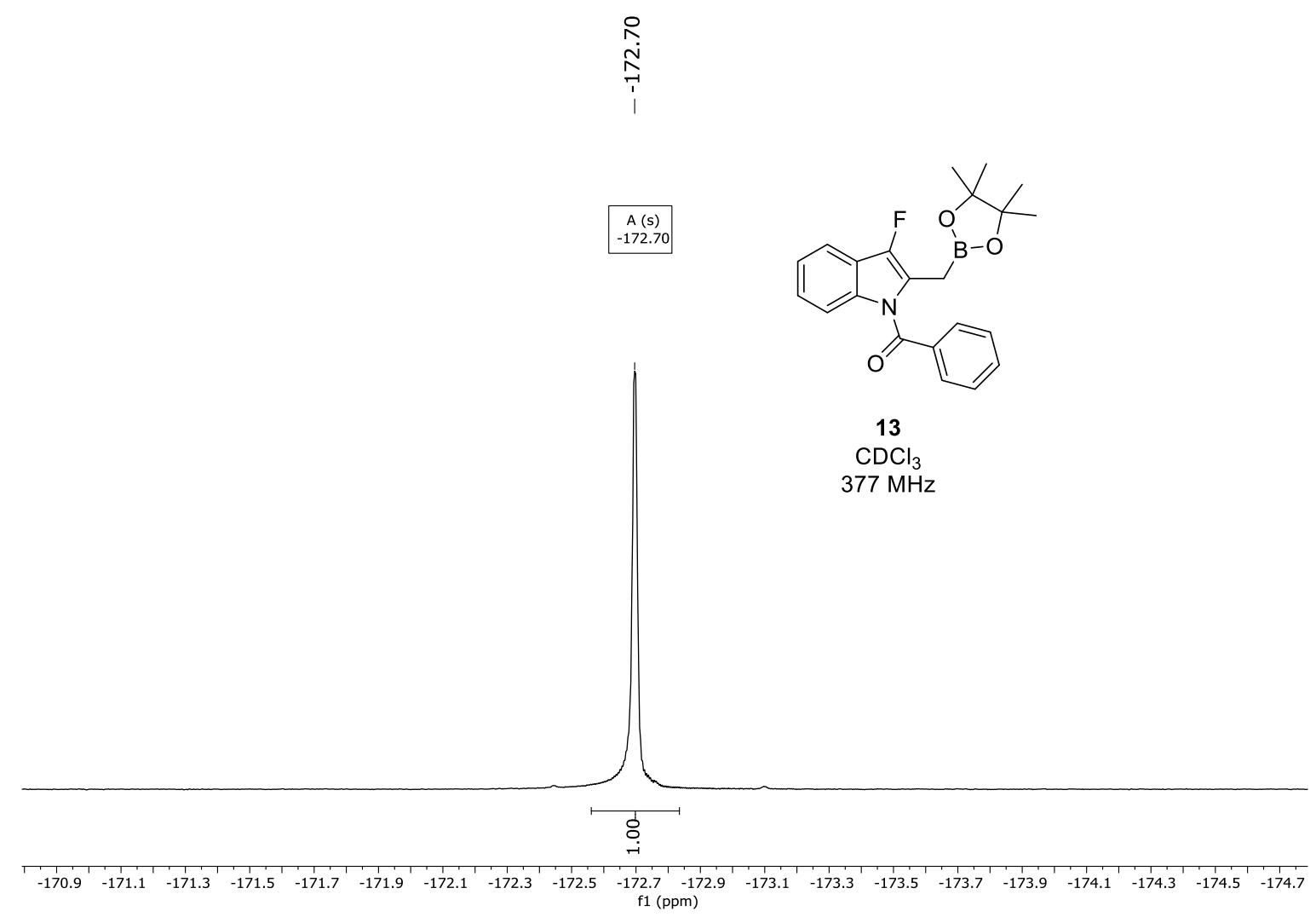

